Arbeitsbereich Politische Theorie und Ideengeschichte

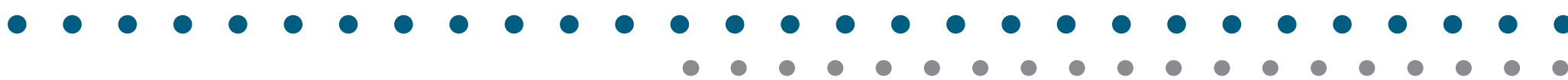

Center for Political Theory \& History of Political Ideas

Valentin Dyckerhoff

\title{
Behinderung und Gerechtigkeit
}

Demokratische Gleichheit

für die gerechtigkeitstheoretische Inklusion von Menschen mit Schädigungen auf der Basis eines interaktionistischen Modells von Behinderung

Working Paper Nummer 2 
In der Working-Paper-Reihe stellt der Arbeitsbereich Politische Theorie und Ideengeschichte des Otto-Suhr-Instituts Arbeiten vor, die sich mit aktuellen Debatten und diskussionswürdigen Begriffen dieses Themenfeldes auseinandersetzen. Die aus herausragenden Abschlussarbeiten hervorgegangenen Beiträge sollen jungen Wissenschaftlern ein Forum für Veröffentlichung bieten und Außenstehenden einen Einblick in aktuelle Forschung im Kontext des Arbeitsbereichs gewähren.

Die Working Papers können Sie auf der Homepage des Arbeitsbereiches für Politische Theorie und Ideengeschichte herunterladen.

Eine Druckversion können Sie unter dgrothe@zedat.fu-berlin.de im Sekretariat bei Frau Grothe-Haaser anfordern.

(C) 2013 beim Autor: Valentin Dyckerhoff

Dyckerhoff, Valentin (2013): Behinderung und Gerechtigkeit. Demokratische Gleichheit für die gerechtigkeitstheoretische Inklusion von Menschen mit Schädigungen auf der Basis eines interaktionistischen Modells von Behinderung. Working Paper Nr. 2, April 2013

Arbeitsbereich Politische Theorie und Ideengeschichte, Freie Universität Berlin

ISSN 2196-0968 (Print)

ISSN 2196-0976 (Internet)

Freie Universität Berlin

Arbeitsbereich Politische Theorie und Ideengeschichte Herausgeber: Prof. Dr. Gerhard Göhler, Prof. Dr. Bernd Ladwig, Prof. Dr. Klaus Roth Fachbereich für Politik- und Sozialwissenschaften

Otto-Suhr-Institut für Politikwissenschaft Ihnestr. 22, 14195 Berlin, Deutschland Phone: +49(0) 3083854 625, Fax: +49(0) 3083852101 dgrothe@zedat.fu-berlin.de 


\title{
Behinderung und Gerechtigkeit
}

Demokratische Gleichheit für die gerechtigkeitstheoretische Inklusion von Menschen mit Schädigungen auf der Basis eines interaktionistischen Modells von Behinderung

Valentin Dyckerhoff

\begin{abstract}
Das vorliegende Research Paper beschäftigt sich mit der Frage nach der akzeptabelsten Konzeption einer Metrik und Verteilungsregel für die gerechtigkeitstheoretische Inklusion von Menschen mit physischen und geistig-psychischen Einschränkungen auf der Grundlage eines angemessenen wissenschaftlichen Modells von Behinderung. Es wird argumentiert, dass ein Modell mit interaktionistischen Grundzügen, das die konstitutive Rolle sowohl individueller Zustände (,Schädigungen`) als auch befähigender und behindernder Kontexte betont, das komplexe Phänomen Behinderung am besten widerspiegelt. Ausgehend von einer Kritik an John Rawls Ansatz „Gerechtigkeit als Fairness“, den ,glücksegalitaristischen Positionen“ von Ronald Dworkin und Richard Arneson und den Capability-Ansätzen von Amartya Sen und Martha Nussbaum wird konstatiert, dass eine normativ sparsame Variante der Capability-Metrik mit einer Suffizienzregel der Verteilung - wie sie Elizabeth Andersons Ansatz „Demokratischer Gleichheit“ darstellt - die momentan akzeptabelste Grundlage für die gerechtigkeitstheoretische Berücksichtigung von Behinderung darstellt.
\end{abstract}

\section{Zum Autor}

Valentin Dyckerhoff hat in Edinburgh und Berlin Politikwissenschaft mit dem Schwerpunkt politische Philosophie und Theorie studiert, sich dabei neben der Materie Behinderung u.a. mit den Themen Entwicklungszusammenarbeit, Menschenrechte und Klimawandel beschäftigt und sein Studium im Januar 2013 als Diplom-Politologe abgeschlossen. 



\section{Inhaltsverzeichnis}

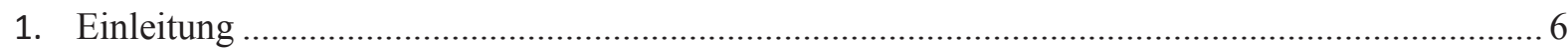







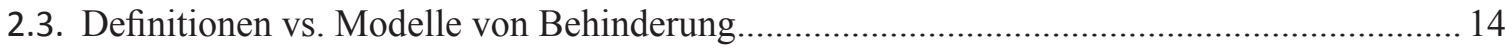

2.4. Die Unterscheidung zwischen ,Schädigung' und Behinderung ............................................ 15

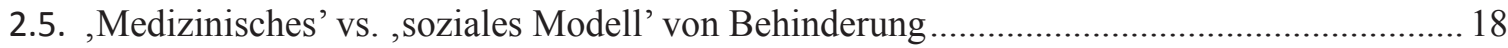



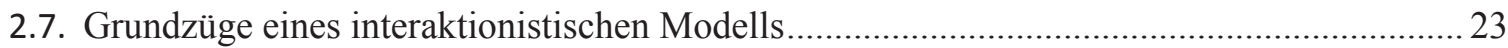



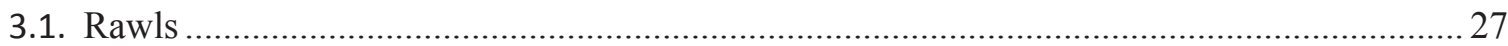

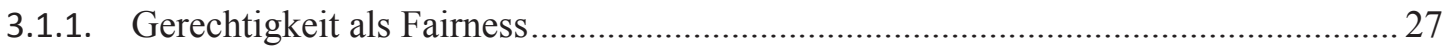

3.1.2. Die Gesellschaft als ein System fairer Kooperation: Öffentlichkeit, Reziprozität und rationale Vorteile .................................................... 28

3.1.3. Freie und gleiche Bürger:

Über eine Lebensspanne voll kooperierende Personen ............................................ 29

3.1.4. Die akzeptabelste Konzeption:

Zwei Prinzipien der Gerechtigkeit und die Metrik der Grundgüter ............................ 30

3.2. Die Kritik an Rawls im Hinblick auf die Inklusion von Menschen mit Schädigungen .......... 32

3.2.1. Reziprozität, rationale Vorteile und Behinderung....................................................... 32

3.2.2. Normal kooperierende Personen mit zwei moralischen Vermögen.............................. 34

3.2.3. Der vergleichende Index der Grundgüter und die am wenigsten Begünstigten .......... 37

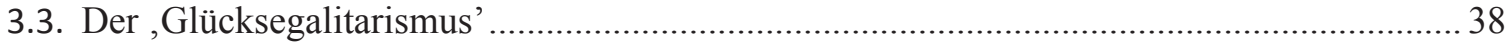

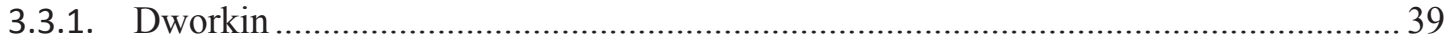

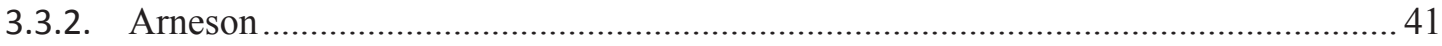

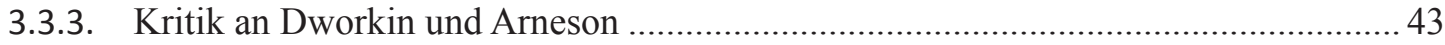

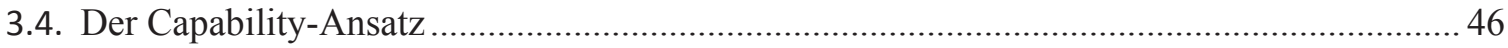

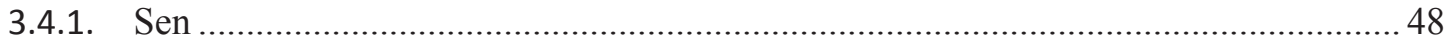

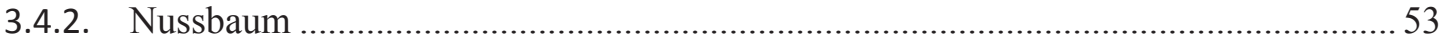

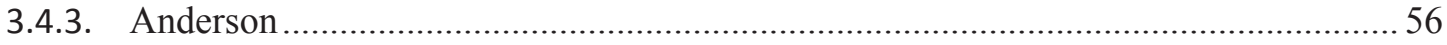

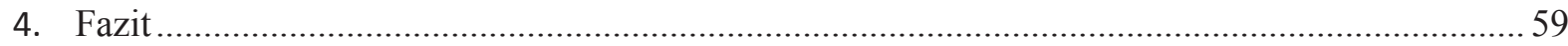

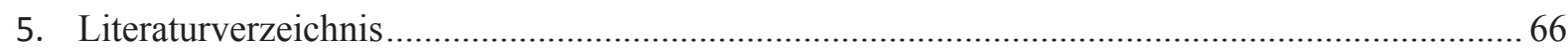




\section{Einleitung ${ }^{1}$}

In einem Artikel für die Zeitschrift Ethics schreibt der US-amerikanische Philosoph Lawrence Becker 2005: „The adequacy of a theory of distributive justice is now measured partly by its success in dealing with justice for the disabled. It was not always so. Up to and including Rawls, no major theory of justice in the Western philosophical tradition made disability a prominent issue“ (Becker 2005: 9).

John Rawls hat in der letzten systematischen Fassung seines Gerechtigkeitsansatzes, Justice as Fairness, die grundlegende Frage nach Gerechtigkeit folgendermaßen formuliert: „What is the most acceptable political conception of justice for specifying the fair terms of cooperation between citizens regarded as free and equal and as both reasonable and rational, and (we add) as normal and fully cooperating members of society over a complete life, from one generation to the next?" (2003: 7f.).

In der Konzeptualisierung von Bürgern als frei und gleich werde von verschiedenen Eigenschaften der sozialen Welt abstrahiert und in einigen Hinsichten idealisiert. Solch abstrakte Konzepte seien nötig, so Rawls: „[T]hey are used to gain a clear and uncluttered view of a question seen as fundamental by focusing on the more significant elements that we think are most relevant in determining its most appropriate answer" (ebd.: 8).

Die vorliegende Arbeit folgt im Gegensatz zu Rawls der Überzeugung, dass Behinderung als ein Phänomen, das beinahe jeden Menschen zu einem Zeitpunkt seines Lebens betrifft, eines dieser ,signifikanten Elemente' darstellt, das relevant ist für die Beantwortung der Frage, welches die akzeptabelste Konzeption politischer Gerechtigkeit ist. Die Arbeit soll sich dementsprechend mit der gerechtigkeitstheoretischen Inklusion von Menschen mit Schädigungen beschäftigen ${ }^{2}$.

Es liegt folgende Fragestellung zugrunde: Welche gerechtigkeitstheoretische Metrik (und Verteilungsregel) ist auf der Basis eines angemessenen wissenschaftlichen Verständnisses von Behinderung geeignet, die Rechte und Pflichten von Menschen mit funktionellen und strukturellen Beeinträchtigungen zu inkludieren? Was sind die konzeptionellen Grundzüge eines solchen angemessenen Verständnisses von Behinderung?

In Beantwortung der zweiten Teilfrage soll die These verteidigt werden, dass nur ein Modell mit interaktionistischen Grundzügen, das die konstitutive Relevanz sowohl von persönlichen Zuständen als auch von Eigenschaften des physischen und sozialen Kontextes berücksichtigt, der komplexen Natur des Phänomens Behinderung angemessen ist. Behinderung entsteht weder nur aufgrund von

1 Dieses Research Paper ist eine leicht gekürzte und überarbeitete Version meiner im Herbst 2012 eingereichten Diplomarbeit. Für fachliche und emotionale Unterstützung danke ich von ganzem Herzen Carina Breschke.

2 Als „Schädigung“ wird im hier verteidigten Grundverständnis eines wissenschaftlichen Modells von Behinderung der individuelle Zustand funktioneller und struktureller Beeinträchtigungen bezeichnet, siehe Abschnitt 2.4. 
funktionellen und strukturellen Beeinträchtigungen, noch ausschließlich als Resultat physischer Barrieren oder sozialer Diskriminierung und Marginalisierung.

Im Hinblick auf die erste Teilfrage soll argumentiert werden, dass eine auf normativ relativ sparsamen Annahmen beruhende Variante der Capability-Metrik in Verbindung mit einer Suffizienzregel der Verteilung - wie sie etwa die US-amerikanische Philosophin Elizabeth Anderson verteidigt - die akzeptabelste Konzeption politischer Gerechtigkeit ist, die Rechte und Pflichten von Menschen mit Schädigungen auf der Basis eines interaktionistischen Behinderungsmodells inkludiert. Die Capability-Metrik berücksichtigt, im Gegensatz zu anderen Gerechtigkeitsmetriken, zu welchen Tätigkeiten und Lebensweisen Menschen mit verschiedenen Eigenschaften in partikularen Kontexten durch die Verteilung bestimmter moralisch wertvoller Güter effektiv befähigt werden.

Eine Suffizienzregel der Verteilung begrenzt auf der einen Seite im Konflikt um endliche zu verteilende Güter die Bereitstellung solcher Güter auf eine - im Hinblick auf objektive Kriterien bestimmbare - hinreichende Schwelle. Auf der anderen Seite setzt eine Suffizienzregel zumindest bis zum Erreichen dieser substanziellen Schwelle keine potenziell stigmatisierenden Urteile über die relative Position einzelner Individuen zueinander in einer umfassenden Rangordnung mehr oder weniger begehrenswerter Bündel interner und externer Ressourcen voraus.

Die Arbeit folgt zu diesem Zweck folgendem Aufbau: Im ersten Teil der Arbeit soll das Phänomen und der Begriff Behinderung wissenschaftlich handhabbar gemacht werden (2). Es wird zunächst eine Skizze des empirischen Hintergrunds des Phänomens Behinderung als potenzielle Dimension sozialer Benachteiligung und Marginalisierung in der Europäischen Union (EU) und weltweit vorgenommen (2.1). Im Anschluss wird differenziert, dass Behinderungen auf einer großen Vielfalt von Schädigungen beruhen können - einige Dimensionen sind gerechtigkeitsrelevant (2.2). Darauf aufbauend wird argumentiert, dass angesichts dieser dargestellten Komplexität des Phänomens Behinderung ein angemessenes Verständnis auf einem wissenschaftlichen Modell beruhen muss und nicht auf einer einheitlichen Definition (2.3). Es wird dargestellt, dass die in der wissenschaftlichen Literatur ernsthaft diskutierten Modelle von Behinderung analytisch sinnvoll mindestens die Komponente der Schädigung (bzw. ,impairment') vom übergeordneten Konzept Behinderung unterscheiden (2.4). Ausgehend von einer in der Literatur verbreitet vorgenommenen idealtypischen Unterscheidung zwischen einem so genannten ,medizinischen' und einem ,sozialen Modell' wird argumentiert, dass ein angemessenes Modell von Behinderung das Phänomen weder auf den individuellen $\mathrm{Zu}$ stand der Schädigung reduzieren sollte, noch ausschließlich auf Aspekte des physischen und sozialen Kontexts (2.5). Extremeren Positionen des sozialen Modells, die Behinderung als auf menschlichen Urteilen beruhende soziale Konstruktion verstehen, wird entgegnet, dass Schädigungen epistemologisch objektiv sind und sich in der Regel auf ontologisch objektive Zustände beziehen (2.6). Schließ- 
lich werden die Ergebnisse der Auseinandersetzung mit dem Phänomen und Begriff Behinderung in Grundzügen eines interaktionistischen Modells von Behinderung zusammengeführt (2.7).

Der zweite Teil der Arbeit befasst sich mit der Darstellung und Kritik verschiedener gerechtigkeitstheoretischer Positionen und der jeweiligen Behandlung des Phänomens Behinderung sowie den Implikationen für die theoretische Inklusion von Menschen mit Schädigungen auf der Basis eines interaktionistischen Grundverständnisses (3.). Im ersten Abschnitt dieses Teils wird John Rawls Konzeption von Gerechtigkeit als Fairness auf die für das Thema relevanten Aspekte zugespitzt (3.1). Diese werden im Anschluss als zu anspruchsvoll und effektiv exkludierend kritisiert, vor allem im Hinblick auf Menschen mit schweren Schädigungen (3.2). Der von Elizabeth Anderson so benannte ,Glücksegalitarismus' wird daraufhin als eine Antwort auf das bei Rawls diagnostizierte Problem der fehlenden Berücksichtigung unverdienter Einschränkungen der Lebensaussichten aufgrund ,interner Merkmale' interpretiert (3.3). Als Vertreter der ,glücksegalitaristischen' Position werden im Folgenden Ronald Dworkins Ansatz der Ressourcengleichheit (3.3.1) und Richard Arnesons Konzeption gleicher bzw. prioritär verteilter Aussichten auf Wohlergehen (3.3.2) vorgestellt. Es wird beanstandet, dass beide gerechtigkeitstheoretischen Ansätze das Phänomen der Behinderung wesentlich auf die Komponente der Schädigung reduzieren (3.3.3).

In einem nächsten Schritt wird der Capabilities-Ansatz als eine alternative Antwort auf die Kritik an Rawls präsentiert, die die interaktionistische Natur des Phänomens Behinderung besser berücksichtigen könne (3.4.). Amartya Sens Position gleicher Capability-Sets (3.4.1) wird ähnlich wie Dworkin und Arneson vorgeworfen, Behinderung zu einseitig als Beeinträchtigung individueller $\mathrm{Zu}-$ stände zu behandeln und darüber hinaus zu wenig konkrete Kriterien der Gerechtigkeit zu spezifizieren. An der aristotelisch inspirierten Interpretation der Capabilities durch Martha Nussbaum (3.4.2) wird ähnlich wie an der Position von Rawls der effektive Ausschluss von Menschen mit schweren Schädigungen bemängelt - in diesem Fall aufgrund zu anspruchsvoller normativer Vorstellungen menschlichen Gedeihens. Zuletzt wird schließlich der Capabilities-basierte Ansatz, demokratischer Gleichheit' von Elizabeth Anderson vorgestellt (3.4.3). Dieser sei aufgrund seiner normativ sparsameren und als funktionell demokratisch konzipierbaren Annahmen sowie der nicht-relationalen, soziales Stigma vermeidenden Kennzeichen die zur Zeit akzeptabelste Konzeption politischer Gerechtigkeit, die die Inklusion von Menschen mit Schädigungen auf der Basis eines Modells von Behinderung mit interaktionistischen Grundzügen berücksichtigen kann. Abschließend wird resümierend ein Fazit gezogen (4.). 


\section{Behinderung}

In diesem Abschnitt soll eine wissenschaftliche Klärung des Phänomens und des Begriffs Behinderung vorgenommen werden. Wenn, wie im Weiteren argumentiert, ein angemessenes Modell von Behinderung aus verschiedenen für soziale Gerechtigkeit relevanten Komponenten besteht - sowohl individuellen als auch solchen des physikalischen und sozialen Umfelds - dann sollte eine Gerechtigkeitstheorie die Möglichkeit bieten, auf diese verschiedenen Komponenten zu sprechen zu kommen (vgl. Vehmas 2012: 301).

\subsection{Empirischer Hintergrund}

Behinderung ist eine weltweit sehr verbreitete Dimension potenzieller sozialer Benachteiligung. Sie gehört z.B. laut dem Gemeinsamen Bericht der Europäischen Kommission und des Rates zu einem der dominanten Risikofaktoren für Armut und soziale Ausgrenzung (EU 2004: 35). Hinsichtlich sozialer Exklusion und Stigmatisierung trifft das über soziale Klassen und Schichten hinweg zu (Maschke 2008: 272). Auch wenn der Status als Mensch mit Schädigung nicht mit Armut und sozialer Marginalisierung gleichgesetzt werden sollte (z.B. Hollenweger 2003), lässt sich diese Erkenntnis statistisch z.B. in Hinsicht auf Bildung, Zugang zum Arbeitsmarkt und Einkommen sowie in Bezug auf die Beeinträchtigung von sozialen Kontakten weitgehend untermauern (Maschke 2008: 115f.).

Es ist nicht unproblematisch belastbare Statistiken zu erstellen. Das liegt auf der einen Seite daran, dass es in den meisten Ländern (glücklicherweise) keine ,Meldepflicht' oder Ähnliches für Behinderungen gibt. Auf der anderen Seite beruhen statistische Erhebungen in verschiedenen Ländern auf unterschiedlichen Definitionen und Methodiken. Daher kommt z.B. die United Nations Disability Statistics Database (UN DISTAT 2011), die verschiedene nationale Untersuchungen aggregiert, auf kaum vergleichbare Zahlen zwischen den Ländern (Bickenbach 2012b: 58, vgl. Becker 2005: 9). Eine für Deutschland berücksichtigte Umfrage von 1992 kommt z.B. auf 8,4\% von Menschen mit Schädigungen, in Norwegen 1991 auf über 30\% und im Gazastreifen für 1996 auf 2,1\% (UN DISTAT 2011). Aufgrund der fehlenden Einheitlichkeit ist die Datenbank noch kaum als statistisches Instrument zu gebrauchen, die Internationale Klassifikation der Funktionsfähigkeit, Behinderung und Gesundheit (kurz ICF, WHO 2001; deutsche Übersetzung DIMDI 2005) soll erst noch implementiert werden.

Auf Grundlage einer der international belastbarsten Quellen, dem World Health Survey der Weltbank und WHO von 2004, der auf der ICF basiert, kann man extrapoliert grob von ca. 15\% der Weltbevölkerung mit signifikanten Beeinträchtigungen ausgehen bzw. vermutlich mehr als einer Milliarde Menschen (WHO Report on Disability 2011: 29, 261). Diese Zahl wird eher zunehmen, unter anderem wegen weltweit durchschnittlich alternder Bevölkerungen und der Zunahme von chronischen 
Krankheiten, die das Auftreten und die Charakteristika von Behinderungen beeinflussen (ebd.: 262, Maschke 2008: 19).

Es gibt bezüglich der Inzidenzen von Behinderung weltweit große Disparitäten, sowohl zwischen Ländern des globalen Südens und Nordens als auch innerhalb dieser jeweiligen Gruppen. Der World Health Survey, der auf einer repräsentativen Umfrage der über 18-Jährigen in 59 Ländern beruht, nennt 11,8\% Betroffene in Ländern mit hohem durchschnittlichen Pro-Kopf-Einkommen und $18 \%$ für solche mit niedrigem Einkommen (gemessen an einer einigermaßen willkürlichen Schwelle von einem Pro-Kopf-Bruttonationaleinkommen von jeweils über und unter ca. 10.000 US\$) (WHO 2011: 27, 30).

In der EU sind es 10 bis 15\%, die als Menschen mit Schädigungen klassifiziert werden oder sich selbst in Umfragen so bezeichnen (Maschke 2008: 76). Auch innerhalb der EU gibt es aufgrund unterschiedlicher nationaler Klassifizierungen sehr unterschiedliche Angaben: Im British Household Panel Survey wird z.B. eine deutlich weitere Definition verwendet, weshalb die Werte hier ausscheren: im Jahr 2001 16,4\% im Gegensatz zu 10,2\% in Deutschland und 10,4\% in Frankreich (ebd.).

Laut den Statistiken sind so genannte, vulnerable Gruppen', also z.B. Frauen, Menschen in den unteren Einkommenssegmenten und ältere Menschen, überproportional betroffen (WHO 2011: 27). Es kann hier von Effekten intersektionaler Dimensionen sozialer Benachteiligung ausgegangen werden. Menschen mit Schädigungen haben durchschnittlich niedrigere Bildungsabschlüsse, einen weniger guten Zugang zu Arbeitsmärkten, niedrigere Einkommen und weisen ein höheres Risiko für Armut auf (Maschke 2008: 82ff.). Der Zusammenhang zwischen Armut und Behinderung besteht dabei in beide Richtungen: Sowohl vergrößert Armut das Risiko von Schädigungen, die zu Behinderungen führen können, als auch Behinderungen das Risiko, in relativer Armut zu leben (WHO 2011: 10). Die durchschnittlichen Einkommen bleiben auch nach Berücksichtigung von Sozialtransfers deutlich unter dem Bevölkerungsdurchschnitt (Maschke 2008: 116).

Der Anteil von Menschen mit Schädigungen ist dabei entscheidend vom jeweiligen Kontext abhängig. In vielen Ländern des globalen Südens gibt es z.B. kaum Zugang zu grundlegenden Hilfsmitteln. Das wiederum bedingt insgesamt schlechtere Aussichten, was Bildung, Zugang zum Arbeitsmarkt und Gesundheitsversorgung angeht. Ob man z.B. in Australien Paraplegiker ist oder in Kamerun, wo jeder zweite betroffene Landbewohner keinen Zugang zu einfachsten Rollstühlen hat, ist von entscheidender Relevanz für die eigene Funktionsfähigkeit und assoziierte Lebensaussichten (Shakespeare 2012: 272, 278).

Behinderung ist international ein signifikanter Kostenfaktor, das Ausmaß ist jedoch schwer zu quantifizieren. Der 2011 erstmals erschienene World Report on Disability der WHO unterscheidet zwischen verschiedenen Dimensionen von Kosten, etwa solchen, die den Betroffenen selbst, ihrem familiären und sozialen Umfeld, den Arbeitgebern und Sozialsystemen entstehen (WHO 2011: 42ff.). 
Die direkten Kosten, die durch Sozialversicherungen und Sozialhilfe entstehen, sind dabei noch am ehesten bezifferbar und werden für 2007 mit 1,2\% öffentlicher Sozialausgaben in OECD-Ländern für 6\% der berücksichtigten Bevölkerung angegeben (ebd.: 43). Behinderung ist damit innerhalb der OECD der drittgrößte eigenständige Posten nationaler Sozialbudgets, nach Alter und Gesundheit (Maschke 2008: 48).

Darüber hinaus entstehen zusätzlich indirekte Kosten: in ökonomischer Hinsicht etwa bezüglich schwer errechenbarer Produktivitäts- und Steuerverluste sowie nicht zuletzt umfangreiche nicht-ökonomische Kosten sozialer Isolation und Marginalisierung (WHO 2011: 44). Gleichzeitig stellen die Autoren des World Disability Reports heraus, dass diese Kosten in inklusiveren „,settings“ deutlich reduziert werden könnten (ebd.: 42).

In sämtlichen EU-Ländern wird eine Mischung aus kompensationsorientierter, rehabilitationsorientierter und partizipationsorientierter Behindertenpolitik verfolgt, mit jeweils unterschiedlichen Schwerpunkten in einzelnen Ländern (Maschke 2008: 169). Der Einfluss der EU im Bereich der Behindertenpolitik ist anhaltend relativ gering (ebd.: 170). Rehabilitations- und Partizipationspolitiken nehmen insgesamt zu, kompensatorische Politiken aufgrund niedrigerer Effektivität und Effizienz eher ab, nicht zuletzt wegen des zunehmenden finanziellen Drucks auf nationale Sozialbudgets (ebd. 269f.).

Insgesamt haben aber Faktoren wie die sozioökonomische Struktur der Bevölkerung, das Wirtschaftswachstum und Kontextbedingungen wie sozialstaatliche Institutionen (v.a. das Bildungs- und Gesundheitssystem, die gesetzliche Regulierung des Arbeitsmarkts) empirisch einen größeren Einfluss auf die Lebenssituation von Menschen mit Schädigungen als der herrschende Ansatz der nationalen Behindertenpolitiken (ebd.: 177). Die soziale Lage von Menschen mit Schädigungen ist dabei stark von der gesamtwirtschaftlichen Entwicklung und der Integration in den Arbeitsmarkt abhängig (ebd.: 190).

Die hier angeführten Zahlen beruhen zudem grundlegend darauf, Behinderung als einen klar unterscheidbaren Zustand zu kennzeichnen: Ein Mensch ist entweder von Behinderung betroffen oder er ist es nicht. Diese Charakterisierung ist jedoch nicht unumstritten, manche Theoretiker konzeptualisieren Behinderung eher als Kontinuum oder sogar als universelle menschliche Eigenschaft (Bickenbach 2012a: 79, Nussbaum 2007: 125, zentral: Zola 1989: 401ff.). Vor allem für PolicyZwecke müssen zwar in der Regel klare Grenzen gezogen werden, wer etwa im pädagogischen oder rechtlichen Sinn als Mensch mit Behinderung gelten kann - dies ist aber eine prinzipiell politisch verhandelbare Grenze (Bickenbach 2012a: 15).

Aus dieser empirischen Skizze folgt natürlich zunächst nichts direkt für die normative Ebene der Auseinandersetzung. Es sollte nur angedeutet werden, dass der Komplex Behinderung ein relevanter 
Faktor weltweiter sozialer Benachteiligung ist und das Ausmaß der Benachteiligung von den jeweiligen politischen und sozioökonomischen Kontexten abhängig ist.

\subsection{Die Vielfalt der Formen von Schädigungen}

Behinderung ist eine komplexe Dimension potenzieller sozialer Benachteiligungen - sie kommt auf verschiedene Weisen zustande, manifestiert sich in diversen Formen und wird durch vielfältige intervenierende Faktoren beeinflusst (vgl. z.B. WHO 2011: 7ff.). Einige Unterscheidungen in diesen Dimensionen sind relevant für Ansätze sozialer Gerechtigkeit. Die Vielfalt der Manifestationsformen von Behinderung erschwert darüber hinaus das Unterfangen, zu bestimmen, was begrifflich-analytisch zum Phänomen ,Behinderung' insgesamt gehört - im Gegensatz zu Charakteristika, die nur kontingent oder nur auf bestimmte Ausprägungen von Behinderung zutreffen.

In der Literatur wird oft unzureichend zwischen verschiedenen Formen von individuellen $\mathrm{Zu}$ ständen differenziert, die zu Behinderung führen können - den so genannten ,Schädigungen' (siehe 2.4). Meistens beziehen sich Beispiele oder implizite Annahmen auf (angeborene) körperliche Behinderungen (Eurich 2011: 35). Als exemplarisch können die später diskutierten gerechtigkeitstheoretischen Beiträge von Arneson und Dworkin gelten. Blindheit, Verlust der Extremitäten oder schwere Stoffwechselerkrankungen gelten meist als Grenzfälle, die die Sinnhaftigkeit exakter Gleichverteilungen bestimmter grundlegender Güter in Frage stellen sollen - weil solche Schädigungen stets notwendig zu schweren Einschränkungen des Wohlergehens führen würden. Solch eine Auffassung kann dazu führen, dass Menschen mit Schädigungen generell als defizitär dargestellt werden (Wasserman 2001: 237).

$\mathrm{Zu}$ einigen grundlegenden Unterschieden gehört etwa die Frage, ob die einer Behinderung zugrunde liegende Schädigung angeboren oder, erworben' ist. Nach der Lektüre philosophischer Auseinandersetzungen zum Thema Behinderung, die sich zum Beispiel umfangreich mit dem Thema der moralischen Legitimität der Abtreibung von Föten mit Schädigungen beschäftigen (siehe z.B. die ,Singer-Debatte', Buchanan et al. 2000, viele Beiträge in Wasserman et al. 2005), kann es überraschen, dass in Deutschland und EU-weit nur ca. 4-5\% der Behinderungen zugrunde liegenden Schädigungen angeboren sind (BMAS 2011).

Es kann darüber hinaus differenziert werden, ob der betroffene Mensch leichte, schwere oder mehrfache Schädigungen aufweist, und ob mit den vorhandenen Schädigungen Schmerzen einhergehen. Nicht jede Schädigung ist schon notwendig ein Übel, wie im nächsten Abschnitt ausgeführt werden soll (Schramme 2003a).

Eine der für Gerechtigkeitstheorien grundlegendsten Unterscheidungen ist darüber hinaus die zwischen ausschließlich körperlichen und geistigen oder psychischen Schädigungen. Diese Diffe- 
renzierung wird zumindest in großen Teilen der Sekundärliteratur zu Gerechtigkeitstheorien berücksichtigt, die sich auch mit dem Thema Behinderung beschäftigen. Auf der einen Seite entstehen in diesem Zusammenhang zusätzliche begriffliche Schwierigkeiten: Sind geistige Schädigungen z.B. grundsätzlich von psychischen zu unterscheiden (vgl. Schramme 2003b: 54) ${ }^{3}$ ? Auf der anderen Seite haben die assoziierten Formen von Behinderung weitreichende Implikationen für die gerechtigkeitstheoretische Inklusion betroffener Menschen. Wie im Kapitel zu Gerechtigkeitstheorien noch näher erläutert werden soll, verursachen ausschließlich körperliche Behinderungen zum Beispiel Probleme für das Verständnis einer Gesellschaft als System fairer Kooperation und die damit verbundene Erwartung gegenseitiger rationaler Vorteile. Geistig-psychische Schädigungen hingegen stellen noch grundlegender die Voraussetzungen liberaler Gerechtigkeitstheorien infrage, die Subjekte zu unterschiedlichen Graden als autonomiefähige Personen kennzeichnen, die z.B. Vorstellungen vom Guten entwickeln und selber einen Gerechtigkeitssinn haben.

Weiterhin kann man zwischen verschiedenen Verlaufsformen von Schädigungen und assoziierten Behinderungen unterscheiden. Die Differenzierung zwischen relativ statischen Zuständen (z.B. Querschnittslähmung), progedienten (z.B. die verschiedenen Formen von Muskeldystrophie, unberechenbar: Multiple Sklerose) und episodischen Verläufen (z.B. Schizophrenie) (vgl. Kastl 2010: 53) kann bestimmte Ansätze von Gerechtigkeitstheorien vor Probleme stellen. So genannte ,starting


einem bestimmten Zeitpunkt, dem Anfang eines Lebens von Gerechtigkeitssubjekten, herstellen wollen, können solchen Unterschieden nicht ohne Weiteres gerecht werden.

Eine mögliche Unterteilung in sichtbare und äußerlich zunächst unsichtbare Schädigungen macht schließlich deutlich, dass bestimmte Konzeptualisierungen von Behinderung unzureichend oder einseitig sind ${ }^{4}$. Schädigungen wie Diabetes haben z.B. in der Regel keinen „Stimuluscharakter“ für andere Menschen (so Cloerkes 2007: 7) und das Leiden an Diabetes kann, wenn überhaupt, nur auf umständliche Weise auf die Diskriminierung durch andere Menschen oder auf soziale Strukturen zurückgeführt werden.

Ein modellhaftes Verständnis von Behinderung muss auf diese Diversität von Manifestationsformen eingehen können und einen analytischen Kern treffen, der allen diesen Formen gemein ist.

3 Die Nomenklatur ist umstritten. Der deutsche Philosoph Thomas Schramme argumentiert, die Unterscheidung zwischen geistigen und psychischen Schädigungen sei nicht sinnvoll, weil die Verwendung der Qualifizierung, geistig' meist dann vorgenommen werde, wenn organische Grundlagen bekannt seien und die Unterscheidung so einer Fortsetzung des Körper-Geist-Dualismus gleichkomme (2003b: 54, Fn. 3). „Psychisch“ scheint mir jedoch als Kategorie z.B. für nur geminderte kognitive (im Gegensatz zu auch affektiven) Leistungen recht eng und weit entfernt vom Alltagsgebrauch. Ich werde daher im Weiteren der Lesbarkeit halber von geistig-psychischen Schädigungen sprechen. In jedem Fall ist Schramme darin zuzustimmen, dass die Qualifikation „seelisch“" veraltet ist (ebd., so aber z.B. im SGB IX §2, s.o.; ÜRMB, UN 2006). Auch die Bezeichnung „Person mit einem Problem im Lernen“ der ICF (DIMDI 2005: 171) scheint mir nicht treffend und grenzwertig euphemistisch, weil es sich etwa in Fällen affektiver Einschränkungen bei manchen Formen des Autismus schlicht nicht um Lernprobleme handelt.

4 Dazu ausführlich: Davis, Ann (2005): Invisible Disability, in: Ethics, Band 116, Nr.1, S. 153-213. 


\subsection{Definitionen vs. Modelle von Behinderung}

Der amerikanische Rechtsphilosoph Jerome Bickenbach, der unter anderem entscheidend an der Entstehung der letzten WHO-Klassifikation von Behinderung mitgewirkt hat, betont, dass verschiedene Konzeptualisierungen von Behinderung entscheidende Folgen für alle Dimensionen von Behindertenpolitik haben (2012a: 68). Bickenbach charakterisiert die Frage nach einem angemessenen Verständnis von Behinderung als eine der großen immerwährenden Kontroversen im politischen Umgang mit Behinderung (ebd.).

Die Auseinandersetzung um eine definitive, richtige wissenschaftliche Definition von Behinderung ist jedoch letzten Endes fehlgeleitet. Im sozialwissenschaftlichen Zugriff ist für das Verständnis von Behinderung mehr als eine einheitliche Definition nötig (Altman 2001: 97, Bickenbach 2012b: 51). Definitionen sind wissenschaftliche Instrumente, die in verschiedenen Ordnungssystemen bestimmten Zwecken dienen - sie müssen ,fit to purpose“ sein und sollen eine exakte Bedeutung zuweisen (Altman 2001: 98, Bickenbach 2012a: 78 bzw. Nohlen / Schultze 2004: 117). Juristische, medizinische und sozialpolitische Definitionen von Behinderung werden daher sehr unterschiedlich ausfallen, und müssen dies auch, um relevante Aspekte verschiedener Politikfelder aufzugreifen. Klinische Definitionen brauchen z.B. einen klaren Bezug auf gesundheitliche Zustände (Bickenbach 2012b: 52).

Auch innerhalb eines Politikfeldes, wie etwa in der Sozialpolitik, können unterschiedliche Definitionen nötig sein. So können z.B. präventive Antidiskriminierungspolitiken sehr weite bzw. inklusive Definitionen verwenden - oder sogar ganz auf sie verzichten (wie etwa im Fall des deutschen Grundgesetzes oder des bundesdeutschen Allgemeinen Gleichbehandlungsgesetzes und der entsprechenden EU-Richtlinie ${ }^{5}$ ) (Bickenbach 2012a: 74). Kompensatorische oder korrektive Sozialpolitiken hingegen, die einen nicht unwesentlichen Einsatz von Mitteln für einzelne Personen zur Folge haben können, benötigen eine engere bzw. eindeutigere Definition, um das Problem des Missbrauchs bzw. der Überinklusion nicht eigentlich berechtigter Empfänger zu vermeiden. In diesem Sinne ist Behinderung z.B. für die Zwecke des Arbeitsförderungsrechts im dritten deutschen Sozialgesetzbuch (SGB III § 19) enger definiert als im neunten Sozialgesetzbuch zur Rehabilitation und Teilhabe behinderter Menschen (SGB IX $\S 2)^{6}$ (vgl. Felkendorff 2003: 30): Als behindert im ersten Fall gelten nur solche Menschen, deren Aussichten am Arbeitsleben teilzuhaben wesentlich gemindert sind, während es im zweiten Fall alle Menschen sind, deren körperliche oder geistig-psychische Funktion für einen Mindestzeitraum von typischen Zuständen abweichen. Bickenbach zählte 2003 in den USA ganze 67 verschiedene juristische und policy-Definitionen (Bickenbach 2012a: 20). Das soll noch nicht heißen,

5 Grundgesetz Art. 3, Abs. 3 und AGG §1, Abs. 1, zugänglich unter http://www.gesetze-im-internet.de/agg bzw. /gg; Richtlinie 2000/78/EG des Rates, zugänglich unter: http://eur-lex.europa.eu, zuletzt eingesehen: 07.09.2012.

6 Vgl. http://www.gesetze-im-internet.de/sgb_3/index.html, bzw. /sgb_9/index.html, zuletzt eingesehen: 07.09.2012. 
dass dies an sich legitim oder angemessen ist. Definitionen von Behinderung sind natürlich auch in den jeweiligen Politikfeldern umkämpft (Maschke 2008: 30).

Eine Vielzahl von Definitionen von Behinderung für verschiedene Politikfelder ist jedoch nicht per se ein Problem. Bickenbach und die amerikanische Soziologin Barbara Altman sehen in Teilen der Literatur zu Behinderung eher die Schwierigkeit untereinander inkonsistenter Definitionen, und dass partikulare Definitionen zu universalen ,umdeklariert‘ werden (Altman 2001: 97, 101; Bickenbach 2012b: 52f.). Dies führe zu unerwünschten Konsequenzen. Auf der einen Seite sind viele partikulare Definitionen für andere Einsatzfelder ungeeignet. So ist z.B. eine juristische Definition etwa im Sinne des neunten deutschen Sozialgesetzbuchs (SGB IX § 2) nach zeitlichen Abschnitten und Graden der Behinderung für ein soziologisches Verständnis völlig unzureichend, da dieses auch die Auswirkungen auf die Lebensgestaltung betroffener Individuen berücksichtigen will (Cloerkes 2007: 4). Auf der anderen Seite können untereinander inkonsistente Definitionen zu widersprüchlichen und konfligierenden Politiken und Rechtsprechungen führen.

Eine wissenschaftliche Konzeptualisierung bzw. ein wissenschaftliches Modell kann verschiedene Definitionen generieren und gleichzeitig die Konsistenz derselben untereinander sicherstellen (z.B. Bickenbach 2012b: 53). Ein Modell ist eine theoretische Konstruktion a priori und keine empirische Generalisierung - es ist eine Struktur, die meist unterschiedliche Komponenten in sinnhafte Zusammenhänge bringt und somit hilft, komplexe Phänomene wie eben Behinderung zu verstehen (ebd., vgl. Nohlen / Schultze 2004: 559). Eine Konzeptualisierung ist in diesem Sinne nicht an sich wahr oder falsch - sie kann lediglich mehr oder weniger angemessen, hilfreich oder konsistent sein (Altman 2001: 101). Im Fall des Phänomens Behinderung gibt es im Grunde nur eine Handvoll grundlegender Modelle, die sich darüber hinaus in einigen Hinsichten sehr ähneln (vgl. Bickenbach 2012b: 53).

\subsection{Die Unterscheidung zwischen ,Schädigung' und Behinderung}

In Beträgen zum Thema Behinderung wird zumeist mindestens die Komponente ,Impairment’, im Deutschen meist ,Schädigung', von ,disability“, also dem Phänomen der Behinderung selbst unterschieden - über beinahe alle inhaltlich konkurrierenden Ansichten und Modelle hinweg (zum Überblick Altman 2001, Bickenbach 2012b; darüber hinaus Cloerkes 2007, Shakespeare 2006; so auch in der ICF, WHO 2001 bzw. DIMDI 2005). Es ist eine sinnvolle analytische Trennung - auch in Hinsicht auf Fragen der sozialen Gerechtigkeit - wenngleich sich die Unterscheidung eventuell nicht in allen Fällen aufrechterhalten lässt (Shakespeare 2006: 34). 
Schädigungen werden meist als objektive, auf ein Individuum bezogene und zeitlich relativ stabile ${ }^{7}$ Zustände dargestellt, etwa als „Beeinträchtigungen einer Körperfunktion oder -struktur“ in einem Gesundheitskontext (DIMDI 2005: 16, vgl. Schramme 2003a: 180). Es ist umstritten, ob der Begriff als rein biomedizinische Kategorie oder komplett wertfrei aufgefasst werden kann (kritisch z.B. Shakespeare 2006: 34ff., Wasserman 2001: 222ff.). Natürlich können Schädigungen kausale Folgen bestimmter sozialer Zustände - wie z.B. Mangelernährung - sein, und die Feststellung einer Schädigung erfolgt auf Grundlage mit sozialen Normen verbundener Kriterien. Wie ich in Abschnitt 2.6 darstellen werde, sollten Schädigungen aber als sich auf von Sprechakten unabhängige, entlang epistemologisch objektiver Kriterien feststellbare Zustände beziehend, aufgefasst werden. Die Feststellung einer Behinderung im politischen oder juridischen Sinne muss eine pragmatische Grenze ziehen - auch wenn diese umstritten ist (siehe oben, 2.1).

In irgendeiner Form müssen Schädigungen dabei auch einen Abgleich mit zumindest typischen menschlichen Funktionen zu einem bestimmten Lebensalter einschließen (vgl. z.B. Birnbacher 2009: 190, Schramme 2003a: 187). Ansonsten kommt es zu solch haarsträubenden (wenn auch vermutlich hauptsächlich provokativ gemeinten) Beispielen, wie Michael Oliver sie z.B. in „Understanding Disability“ anführt: Flugzeuge seien im genau selben Sinn eine „Mobilitätshilfe“ für „nicht-Flieger“ wie Rollstühle für „,nicht-Geher“(Oliver 1996: 108). Aber das ist relativ offensichtlich Unsinn. Beide helfen zwar Individuen, die Einschränkungen ihres jeweiligen Körpers zu überwinden, aber es besteht eine grundlegende Asymmetrie: Fliegen ist keine typische menschliche Fähigkeit. Ein Rollstuhl ist für einen schwer gehbehinderten Menschen auch nicht nur einfach eine Fortbewegungsmöglichkeit unter vielen - es ist eine essentielle und oft die einzige Möglichkeit (vgl. Kastl 2010: 51, Shakespeare 2006: 51).

,Schädigung' kann als Begriff im Deutschen etwas missverständlich sein. Zum einen kann er, ebenso wie der manchmal alternativ verwendete Begriff „Versehrung“ (z.B. Kuhlmann 2005, Ladwig 2007), missverständlich einen Schädiger oder Versehrer sowie einen im engeren Sinne organischen Schaden, wie z.B. eine Wunde implizieren - beides ist jedoch in vielen Fällen von Schädigungen nicht der Fall. Zum anderen unterscheidet der Thomas Schramme in seinem Artikel „Behinderung - Absolute oder relative Einschränkung des Wohlergehens?“ zwischen einer deskriptiv-wissenschaftlichen und einer „evaluativ-lebensweltlichen“ Verwendung des Begriffs (Schramme 2003a: 180f.). Während letztere in der Alltagssprache impliziert, dass auch eine Beeinträchtigung des Wohlergehens vorliegt, muss das für erstere nicht notwendig gelten (ebd.). Zumindest in Bezug auf das absolute

7 Im Fall des deutschen Sozialgesetzbuchs z.B. über mindestens sechs Monate (SGB IX $\S 2$ Abs. 1) siehe: http://www. gesetze-im-internet.de/sgb_9/_2.html, zuletzt eingesehen: 07.09.2012. 
Wohlergehen einer Person als interne Bewertung ${ }^{8}$ müsse durch eine Schädigung im deskriptiv-wissenschaftlichen Sinne keine Einschränkung vorliegen, so Schramme (ebd.: 184). Er führt das Beispiel eines zeugungsunfähigen Mannes an, der aber auch keinen Kinderwunsch hat - die Schädigung stellt für diesen Menschen keine erhebliche Einschränkung der Lebensaussichten dar (ebd.). Hinsichtlich des relativen Wohlergehens, also des Vergleichs mit Personen ohne Schädigungen, ist das schwerer vorstellbar - man wird in der Regel die Abwesenheit von Schädigungen vorziehen, wenn man die Wahl hat. Als Ausnahmen, die wohl eher die Regel bestätigen, können eventuell die wenigen Schädigungen gelten, die direkte potenzielle Vorteile mit sich bringen, wie z.B. eine durch Sichelzellenanämie bedingte Malariaresistenz oder in der Vergangenheit ein ansonsten wenig folgenreicher Plattfuß, der bei der Ausmusterung vom obligatorischen Militärdienst behilflich sein konnte.

Da „Schädigung“ jedoch die geläufigste Bezeichnung ist (auch DIMDI 2005) und in Ermangelung einer besseren Alternative werde ich im Sinne dieser Differenzierung im Weiteren ,Schädigung' in der deskriptiv-wissenschaftlichen Dimension verwenden. Diese Klarstellung schlägt sich außerdem in der substanzielleren Feststellung nieder, dass auch in objektiv-externer Hinsicht nicht alle Schädigungen zu Behinderungen führen.

Behinderung sollte hingegen in Grundzügen als evaluativer und relationaler Begriff verstanden werden, etwa als „Einschränkung relevanter Handlungsmöglichkeiten“ vor dem Hintergrund eines physischen und sozialen Kontextes (Schramme 2003a: 180, vgl. Cloerkes 2007: 2). Behinderung ist im Gegensatz zu Schädigungen zumindest nicht ausschließlich ein Gesundheitsthema, auch wenn Menschen mit Schädigungen für Gesundheitsprobleme vulnerabler sein können (z.B. geschwächtes Immunsystem, häufige Operationen etc.) und sie diese oft in komplexeren und kontinuierlicheren Ausmaßen haben (Shakespeare 2012: 274). Wie in Abschnitt 2.1 schon angedeutet und unter 2.7 dargestellt, ist für das Vorliegen einer Behinderung der Kontext ebenso konstitutiv. Ob meine Handlungsmöglichkeiten eingeschränkt sind, hängt unter anderem auch damit zusammen, ob ich im Bedarfsfall einen effektiven Zugang zu Hilfsmitteln habe, wie barrierefrei die mich umgebende Infrastruktur ist und als wie wichtig bestimmte Fähigkeiten in meiner sozialen Umgebung erachtet werden bzw. auch, wie stigmatisiert eine jeweilige Schädigung ist (z.B. HIV / AIDS).

Es ist in diesem Zusammenhang illustrativ, dass der irische Philosoph Richard Hull in seinem Artikel „Defining Disability“ (1998) zwei Beispiele anführt, von denen er meint, dass schwere funktionelle Einschränkungen der Beine hinreichend für bestimmte Benachteiligungen seien. Die betroffenen Menschen könnten z.B. nicht den Mount Everest besteigen, oder ein olympischer Sprinter sein (ebd.: 204). Dem lässt sich entgegnen, dass im Jahr der Veröffentlichung von Hulls Artikel der einsei-

8 Für die Frage, ob Schädigungen und Behinderungen in jedem Fall eine Einschränkung des individuellen Wohlergehens darstellt, ist zunächst die interne Bewertung entscheidend (Schramme 2003a: 180). Damit ist noch keine hedonistische Sichtweise präjudiziert und auch z.B. nicht, ob ausschließlich auf dieser Sichtweise Gerechtigkeitsurteile basieren sollten. 
tig beinamputierte Walise Tom Whittaker und 2006 der Neuseeländer Mark Inglis mit zwei Beinprothesen den Everest bestiegen haben (z.B. Berliner Zeitung, jeweils vom 15.05.1998 und 16.04.2007) und bei den regulären Olympischen Spielen 2012 nach der kontroversen Entscheidung des Internationalen Olympischen Komitees der beidseitig beinamputierte Südafrikaner Oscar Pistorius mit seinen Prothesen im Sprint antrat. Das kann und soll nicht heißen, dass schwere Schädigungen nicht in vielen Fällen auch relevante Handlungsmöglichkeiten ausschließen - auch Körper können behindern (Kuhlmann 2003: 173) und hier wird die Grenze zwischen Schädigung und Behinderung potenziell uneindeutig. Es bedeutet aber, dass die jeweiligen Eigenschaften des Kontexts - z.B. physischer, sozialer und technologischer Art - signifikant beeinflussen, wer in welcher Hinsicht als relevant eingeschränkt gelten kann und wer nicht.

Dem Begriff Behinderung lastet ebenfalls eine gewisse Mehrdeutigkeit an. Eine grundlegende Ambivalenz ist, ob Behinderung ein Partizip oder eine personengebundene Eigenschaft ist (Maschke 2008: 30): Werden Menschen behindert oder hat ein Mensch eine Behinderung? Während ,disability’ im Englischen schon analytisch die ,Unfähigkeit' impliziert, hätte ,Behinderung’ im Deutschen eine „komplexere Syntax“, so Kastl, die dem relationalen Charakter von Behinderung besser Ausdruck verleihen könne (Kastl 2010: 12, vgl. Felkendorff 2003: 29).

Ich werde im Sinne des hier skizzierten Modells mit konstitutiv interaktionistischen Grundzügen von ,Menschen mit Schädigungen’ sprechen. Diese Verwendung soll zum einen verdeutlichen, dass nicht alle Umstände, die zu Behinderungen führen, individueller Natur sind und zum anderen, zu geringerem Maße, klarmachen, dass Schädigungen nicht eine Art, identitärer master status' sind, die Menschen auf eine bestimmte Rolle als defizitäres Wesen o.ä. festlegen. Wenn es sich dabei nicht nur um Euphemismen oder politisch korrekte Ausdrucksweisen handeln soll, können solche Überlegungen wichtig sein, weil sie auf unterschiedliche Modelle von Behinderung verweisen, die auch unterschiedliche Maßnahmen im Rahmen von Behindertenpolitiken zur Folge haben können (vgl. Maschke 2008: 30).

\section{5 ,Medizinisches' vs. ,soziales Modell’ von Behinderung}

In der Literatur zur Modelldebatte wird häufig zunächst sehr idealtypisch zwischen einem so genannten ,medizinischen' und einem ,sozialen Modell' von Behinderung unterschieden (so z.B. Bickenbach 2012a, Eurich 2011, auch Wasserman et al. 2011 im Eintrag der Stanford Encyclopedia of Philosophy - kritisch: Felder 2012, Hollenweger 2003 und vor allem Shakespeare 2006). Sowohl ein medizinisches Modell als auch extreme Varianten eines sozialen Modells sind dem komplexen Phänomen Behinderung jedoch letztendlich nicht angemessen. 
Die Unterscheidung wird verschiedentlich auf den britischen Behindertenaktivisten und -theoretiker Michael Oliver in dessen Buch Social Work with disabled People (1983) zurückgeführt (siehe Shakespeare 2006: 15). Unter dem ,medizinischen' oder manchmal auch individuellen oder „personal tragedy“-Modell (Oliver 1996: 32) wird die Reduktion von Behinderung auf das Individuum und einen medizinisch bzw. organisch feststellbaren Schaden verstanden. Behinderung sei eine stabile individuelle Eigenschaft, die aufgrund einer Abweichung vom normalen (statistisch durchschnittlichen) Zustand ein Übel für den Betroffenen darstellt (Felder 2012: 64) und die vor allem medizinisch korrigiert oder aufgrund dieses Leidens kompensiert werden müsse (ebd.: 68).

Unter dem ,sozialen Modell' indes wird in solch einer ersten Gegenüberstellung meist eine extreme Variante verstanden, die Behinderungen ausschließlich auf soziale Kontextfaktoren zurückführen will. Dazu zählen das britische social model, das Behinderung als Resultat sozialer Unterdrückung versteht und das ursprünglich US-amerikanische minority group model, das Behinderte als eine klar abgrenzbare soziale Minderheit konzeptualisiert, deren Benachteiligungen mit Antidiskriminierungspolitiken und effektiv gleichen Bürgerrechten behoben werden könnten (Shakespeare 2006: 11ff. bzw. 23ff.).

Die Unterscheidung zwischen einem medizinischen und sozialen Modell ist jedoch so stark vereinfacht, dass sie der Komplexität und den graduellen Unterschieden zwischen den tatsächlich bestehenden konkreten Modellen nicht gerecht wird. Shakespeare konstatiert, dass solch eine eindimensionale Dichotomie in den wissenschaftlichen Zugängen nicht existiere (2006: 26). Auf der einen Seite vertrete zumindest kein Sozialwissenschaftler ernsthaft ein ausschließlich medizinisches Modell, auf der anderen Seite stehe hinter dem ,sozialen Modell' eher eine Familie von sozialen Modellen - wie sich im letzten Absatz schon andeutete (ebd.: 9, 15; vgl. Felder 2012: 63). Letztere situieren die Ursachen für Behinderung zu unterschiedlichen Ausmaßen im sozialen Kontext der Individuen. Das medizinische Modell hätte häufig eher die Funktion eines Strohmanns, aus dessen Ablehnung heraus die Vorzüge eines sozialen Modells dargestellt werden sollen (Shakespeare 2006: 18, vgl. Felder 2012: 63).

Ein medizinisches Modell mit den genannten Merkmalen wäre tatsächlich zurückzuweisen, vor allem weil - wie im letzten Abschnitt dargestellt - Schädigungen nicht notwendig zu Behinderungen führen. Es ist aber darüber hinaus von einer „medizinischen Sichtweise“ auf Behinderung zu unterscheiden, die den Anspruch hat, die normative Bewertung von deskriptiv-biologischen Fragen zu trennen (Felder 2012: 63f.). Die Bewertung sollte innerhalb dieser Sichtweise im Idealfall den Betroffenen überlassen werden - der Handlungsauftrag des Mediziners ergibt sich erst aus einem etwaigen subjektiven Leid der Betroffenen. Dass es dabei zu Problemen und Übergriffen kommen kann, widerspricht nicht prinzipiell der Legitimität und Notwendigkeit der medizinischen Sichtweise - so einleuchtend Franziska Felder (ebd.). 
Es sollte dennoch zumindest erwähnt werden, dass die medizinische Handhabung von Behinderung durchaus gewisse die Lebenswelt kolonialisierende Tendenzen haben kann: Als Mensch mit Schädigung begegnen einem bestimmte Experten - Pädagogen, Psychologen, Mediziner - die meist hauptsächlich auf der Grundlage medizinischer Kriterien die Eignung für bestimmte Kontexte, wie etwa einen Schultypus etc., bestimmen. Das schlägt sich auch im deutschen Recht nieder: Dem Anspruch nach beruht die Definition nach SGB IX § 2 zwar auf der ICF, tatsächlich werden die „Grade der Behinderung“ des Gesetzes - etwa zur Bestimmung einer „schweren Behinderung“ und daraus resultierenden sozialstaatlichen Leistungen (SGB IX §2 Abs. 2) - aber ausschließlich unter Verwendung der so genannten „Versorgungsmedizinischen Verordnung ${ }^{69}$ (seit 2008) bestimmt. Der Titel lässt es erahnen: es sind hier nur medizinische Zustände kodiert. Eine tatsächliche Feststellung der Beeinträchtigung von gesellschaftlicher Teilhabe - bzw. Einschränkungen der „Aktivität“ und „Partizipation“ (WHO 2001, DIMDI 2005) würde hingegen „originär sozialwissenschaftliches“ Wissen erfordern (Felkendorff 2003: 32).

Auch extremere Varianten eines sozialen Ansatzes sind dem Phänomen Behinderung nicht angemessen. Solche Ansätze, die ausschließlich auf das physikalische und soziale Umfeld als erklärende Variable für Behinderung rekurrieren - diese Kontextfaktoren also zugleich als notwendig und hinreichend auffassen - stoßen auf eine ganze Anzahl von Problemen, die letztendlich zu ihrer Zurückweisung führen sollten.

Das Hauptproblem eines rein sozialen Ansatzes ist die Rolle und Bedeutung des Körpers sowie von Schädigungen für Behinderung (Vehmas / Mäkelä 2009: 42, vgl. Kastl 2010: 13 und Shakespeare 2006: 34ff.). Es wird von verschiedenen Autoren argumentiert, dass die Konzeptualisierung von Behinderung körperliche Probleme mit einschließen müsse - darunter auch solche, die lange Zeit eher als Anhänger eines rein sozialen Modells galten oder sich als aktive Anhänger von Behindertenbewegungen verstehen, wie etwa Tom Shakespeare selbst (Shakespeare 2006: 39, 55; Vehmas / Mäkelä 2009: 43).

In vielen Fällen von Behinderung, vor allem wenn sie mit schweren oder mehrfachen körperlichen oder auch schweren kognitiven Einschränkungen zusammenhängen, können individuelle funktionelle oder strukturelle Faktoren vermutlich schon hinreichend für relevante Einschränkungen der Aktivität oder Partizipation sein - auch der Körper selbst kann behindern (vgl. Kuhlmann 2003: 174, Wasserman 2001: 229). Andreas Kuhlmann beklagt etwa, dass die völlige Ablehnung medizinischer Förderung Aspekte vieler Betroffener ausblende, wie etwa Schmerzen oder Abhängigkeit von bestimmten Formen der persönlichen Assistenz (2005: 43). Solche Faktoren ließen sich nicht einfach einem „,eindlichen Umfeld“ ankreiden (ebd.).

9 Siehe http://www.gesetze-im-internet.de/versmedv/BJNR241200008.html, zuletzt eingesehen: 12.09.2012. 
Eine ausschließliche Charakterisierung von Behinderung als soziales Phänomen würde darüber hinaus die Identifizierung von Menschen mit Schädigungen und statistische Erhebungen von Inzidenzen erschweren. Solche Prozesse seien aber nötig, um zum einen jene Menschen mit Schädigungen unterstützen zu können, die zu individueller Lebensgestaltung erst ermächtigt werden müssen, und zum anderen, um mit demographischen Daten z.B. nationale Arbeitsmarkt-, Gesundheits- und Sozialpolitiken zu entwerfen (vgl. Kuhlmann 2003: 176).

Extreme Varianten eines sozialen Modells werden zudem von Shakespeare als wenig wissenschaftlich reflektiert kritisiert. Ein ausschließlich soziales Verständnis sei schlecht geeignet, empirische Forschungsansätze anzuleiten. Wenn soziale Unterdrückung schon der dominante Teil der Konzeptualisierung von Behinderung ist, dann seien Wissenschaftler von vorneherein darauf festgelegt, Fälle zu identifizieren, in denen solche Unterdrückung oder soziale Ausgrenzung tatsächlich vorliegt (Shakespeare 2006: 9, 57). Die Schweizer Sonderpädagogin Judith Hollenweger konstatiert ähnlich: Das soziale Modell sei zwar vielleicht als Grundlage emanzipatorischer Politiken und zur Aktivierung und Politisierung von Menschen mit Schädigungen geeignet, nicht aber als Grundlage eines kohärenten wissenschaftlichen Modells (2003: 147) ${ }^{10}$.

Solche Probleme treten exemplarisch in der Konzeptualisierung von Behinderung z.B. der $\mathrm{Di}$ sabled People's International (DPI) zutage. Schädigung (bzw. ,impairment') wird hier gefasst als „the funcional limitation within the individual caused by physical, mental or sensory impairment”, Behinderung hingegen als ,the loss or limitation of opportunities to take part in the normal life of the community due to physical and social barriers" (nach Shakespeare 2006: 14). Während die Definition von Schädigung offensichtlich zirkulär bzw. tautologisch ist, da sie das definiendum selbst enthält, ist in der Charakterisierung von Behinderung keinerlei Rede mehr von Schädigung (ebd.).

Das würde jedoch heißen, dass alle Personen und sozialen Gruppen, die aufgrund physischer oder sozialer Barrieren nicht gleichberechtigt am ,normalen Leben der Gemeinschaft ${ }^{\star}$ teilnehmen können, als behindert gelten könnten. Man kann argumentieren, dass dies unter Berücksichtigung ungleicher Löhne und einer , glass ceiling' für Frauen, Diskriminierung gegenüber ethnischen Minderheiten und sozialer Marginalisierung von armen Bevölkerungsgruppen, nun auf alle diese Gruppen zutreffen müsste. Das ist jedoch nicht einleuchtend: Frauen und arme Menschen sind nicht „behindert", sondern in ungleichen und/oder diskriminierenden Gesellschaften sozial benachteiligt.

Shakespeare bringt den entscheidenden Unterschied zwischen Behinderung und anderen so genannten ,Achsen der Unterdrückung'auf den Punkt: ,[...] impairment often has explanatory relevance in ways that the color of someone's skin, or their sex, or their sexual orientation usually does

10 Es sollte aber angemerkt werden, dass die Vertreter sozialer Modelle und auch Anhänger der disability studies und politisch-emanzipatorischer Teile der Behindertenbewegung entscheidende Beiträge dazu geleistet haben, dass die soziale Dimension in Modellen von Behinderung anerkannt wird und etwa auch Einzug in offizielle Dokumente wie das ÜRMB und die ICF gehalten hat. 
not“ (2006: 42). Ein Mensch mit schweren kognitiven Einschränkungen oder schweren spastischen Lähmungen kann aufgrund seiner individuellen Schädigung auch unter einer richtig verstandenen Auffassung fairer Chancengleichheit z.B. vermutlich eher nicht Chefchirurg werden. Die Hautfarbe oder das Geschlecht eines Bewerbers sollten hingegen in einer gerechten Gesellschaft keinerlei Auswirkungen auf die Eignung für einen solchen Beruf haben. Behinderung als Dimension sozialer Benachteiligung ist insofern kein soziales Konstrukt, wenn damit gemeint ist, dass der Zustand ,irreal“ oder gar ein „Mythos“ sei, bzw. auf vollkommen willkürlichen Festlegungen beruhe (Vehmas / Mäkelä 2009: 45).

\subsection{Behinderung als soziales Konstrukt?}

Sind Schädigungen oder Behinderungen soziale Konstruktionen? Was genau würde das bedeuten und was hätte es für substanzielle Folgen für ein wissenschaftliches Verständnis von Behinderung? Im Rahmen der vorliegenden Arbeit soll ein „kritisch realistisches Verständnis“ (vgl. Shakespeare 2006, Vehmas / Mäkelä 2009: 46) von Behinderung zugrunde gelegt werden. Es beruht auf der Überzeugung, dass der ontologische Status bestimmter physischer und auch sozialer Phänomene, wie etwa die als Schädigungen bezeichneten funktionellen und strukturellen, von Sprechakten unabhängigen Einschränkungen, eine objektive Dimension aufweist ${ }^{11}$. Zumindest extreme sozialkonstruktivistische Positionen, die die von menschlichen Urteilen unabhängige ontologische Realität von Schädigungen und Behinderungen bestreiten, sind insofern zu verwerfen (vgl. generell Schramme 2003b).

Behinderung kann nur in den weniger folgenreichen Hinsichten als soziale Konstruktion verstanden werden, dass die Bewertung einer Schädigung zu einem signifikanten Teil durch die Gesellschaft festgelegt wird, und dass die Normen der Zuschreibung von Behinderung wie alle sozialen Normen gesellschaftlich und kulturell bestimmt sind (Schramme 2003b: 58ff.). Insbesondere der ontologische Status geistig-psychischer Schädigungen und so genannter ,Lernbehinderungen“ ist umstritten (abweichend z.B. Eurich 2011: 212, auch Vehmas 2012 uneindeutig). Dennoch soll im Rahmen der Arbeit u.a. in Anschluss an Thomas Schramme, der sich intensiv mit Aspekten geistig-psychsicher Behinderung auseinandergesetzt hat (siehe ausführlicher z.B. Schramme 2000: „Patienten und Personen - Zum Begriff der psychischen Krankheit"), dafür plädiert werden, dass Zuschreibungen geistigpsychischer Schädigungen und Behinderungen zwar auf bestreitbaren und umkämpften, nicht aber auf willkürlichen sozialen Normen beruhen (Schramme 2003b: 57f.). Das ist insofern für das Argu-

11 Ähnlich z.B. auch Ian Hacking (1999): The Social Construction of What? Hacking argumentiert z.B., dass die Rede von sozialen Konstruktionen meist auch mit einem Werturteil darüber verbunden sei, dass ein von menschlichen Urteilen unabhängiges Verständnis des betreffenden sozialen Phänomens abzulehnende Folgen impliziere (S. 19). Im Abschnitt zur Konstruktion von Behinderung attackiert er u.a. Michael Olivers Konzeption (38f.). 
ment der Arbeit relevant, als dass die konstitutive Relevanz von epistemisch objektiv bestimmbaren Schädigungen, die sich zumindest in der Regel auf ontologisch objektive Zustände funktioneller und struktureller Beeinträchtigungen beziehen, für das Vorliegen von Behinderungen behauptet wird.

\subsection{Grundzüge eines interaktionistischen Modells}

Entsprechend der vorangegangenen Auseinandersetzung sollen also sowohl das ,medizinische Modell' als auch extreme Varianten des sozialen Modells verworfen werden, weil sie dem komplexen Phänomen Behinderung nicht angemessen sind. Stattdessen wird die konstitutive Relevanz von (a) sich auf ontologisch objektive Zustände funktioneller und struktureller Einschränkungen beziehende ,Schädigungen', und (b) physischen und sozialen Bedingungen des Kontexts, für das Ent- und Bestehen von Behinderung herausgestellt.

Ein zunehmender Teil der wissenschaftlichen Literatur konzipiert Behinderung entlang dieser Grundzüge (vgl. Shakespeare 2006: 54ff., darüber hinaus Bickenbach 2012a, 2012b; Buchanan et al. 2000 als „,match“ zwischen Individuum und Kontext, Felder 2012, Hull 1998, Maschke 2008, Vehmas / Mäkelä 2009, Wolff 2009). Sie finden sich darüber hinaus auch im „Übereinkommen über die Rechte von Menschen mit Behinderungen“ (ÜRMB) der Vereinten Nationen (UN) wieder, das als einzigen konzeptuellen Gesichtspunkt in Abschnitt (e) der Präambel anführt, dass die Konvention verabschiedet wird:

,in der Erkenntnis, dass das Verständnis von Behinderung sich ständig weiterentwickelt und dass Behinderung aus der Wechselwirkung zwischen Menschen mit Beeinträchtigungen und einstellungs- und umweltbedingten Barrieren entsteht, die sie an der vollen, wirksamen und gleichberechtigten Teilhabe an der Gesellschaft hindern.“(UN 2006)

Die „Internationale Klassifikation der Funktionsfähigkeit, Behinderung und Gesundheit“ der WHO konzipiert Behinderung als einen „Oberbegriff“ für Schädigungen und Beeinträchtigungen der Aktivität und der Partizipation. Dieser umfasse die „,negativen Aspekte der Interaktion zwischen einer Person (mit einem Gesundheitsproblem) und ihren Kontextfaktoren (Umwelt- und personbezogene Faktoren [sic])“ (WHO 2001, DIMDI 2005: 145f.). Die Klassifikation ist auch kritisiert worden neben eher unangemessenen Anschuldigungen, sie beruhe auf einem ,medizinischen Modell' von Behinderung, vor allem dafür, dass sie zum Teil missverständlich und zu uneindeutig sei (Bickenbach 2012b: 51). Der an den Verhandlungen zur Klassifikation beteiligte Bickenbach kritisiert einleuchtend insbesondere, dass die Unterscheidung zwischen „Aktivität“ und „Partizipation“ (vgl. 2.5) problematisch sei und vor allem Partizipation als „Einbezogensein in eine Lebenssituation“ (DIMDI 2005: 16) viel zu vage bleibe (Bickenbach 2012b: 56, 61). Für die Zwecke des Arguments muss ich mich allerdings nicht auf ein solch konkretes Modell von Behinderung festlegen. Ich möchte mich 
stattdessen auf die oben dargestellte interaktionistische Grundstruktur eines Modells von Behinderung beschränken ${ }^{12}$.

Wenn ein angemessenes Modell von Behinderung aus diesen grundlegenden Komponenten besteht, dann sollte - wie bereits angesprochen - eine Gerechtigkeitstheorie, die Menschen mit Schädigungen inkludieren kann, die Möglichkeit bieten, auf diese verschiedenen Komponenten zu sprechen zu kommen (vgl. Vehmas 2012: 301).

\section{Gerechtigkeit}

Gerechtigkeit ist eine Norm, die beinahe alle sozialen Bereiche potenziell berührt - die erste Aufgabe ist also eine der Eingrenzung des Begriffs. Christoph Horn und Nico Scarano unterscheiden in der Einleitung zu ihrer kommentierten Sammlung klassischer gerechtigkeitstheoretischer Texte sieben institutionenethische Themen innerhalb normativer gerechtigkeitstheoretischer Ansätze und zehn Bereiche, in denen die Qualifizierung gerecht bzw. ungerecht angewandt werden kann (2002: 9f.). Für die Zwecke des Arguments und hinsichtlich der berücksichtigten Beiträge beschränkt sich die vorliegende Arbeit vor allem auf die Themen politischer sowie sozialer und ökonomischer Gerechtigkeit. Das heißt bei Horn und Scarano, dass es um die angemessene Verteilung von Rechten, Freiheiten, Ämtern, Chancen und Ressourcen geht (ebd.). Hinsichtlich der Bereiche, in denen zwischen gerechten und ungerechten Zuständen unterschieden werden kann, beziehen sich die folgenden Abschnitte angemessener Weise hauptsächlich auf soziale Institutionen und Gesellschaftsordnungen.

Gerechtigkeit soll im Weiteren also wesentlich modern als politische Grundnorm verstanden werden (vgl. Ladwig 2004: 119). Die Arbeit macht sich das „Motto“ (so Horn / Scarano 2002: 9) zeitgenössischer Gerechtigkeitstheorien zu eigen, das Gerechtigkeit mit John Rawls als „erste Tugend sozialer Institutionen“ spezifiziert (Rawls 1971: 3). Das heißt für Rawls unter anderem, dass soziale Institutionen, unabhängig von ihrer Effizienz, reformiert oder abgeschafft werden müssen, wenn sie ungerecht sind (ebd.). Politische Gerechtigkeit bezieht sich bei Rawls explizit und auch bei den anderen hier berücksichtigten Ansätzen in weiten Teilen auf die ,, basic structure “ einer Gesellschaft, also das grundlegende System politischer Institutionen (Rawls 2003: 8).

Die Gleichsetzung mit Verteilungsgerechtigkeit, die sich bei Horn und Scarano wiederfindet ist eine moderne Weichenstellung. Aristoteles hat die „Zuteilung von Ehre, Geld und den anderen Dingen, die unter die Mitglieder der Gemeinschaft aufgeteilt werden können“ als eine Form beson-

12 Es ist in diesem Sinne von bestehenden abweichenden und umfassenderen Konzeptionen eines, ,interaktionistischen Paradigmas“ oder „Interaktions-Modells“ abzugrenzen. Diese bei Cloerkes angeführten Konstrukte sind mit Vorstellungen von Behinderung als Resultat eines Prozesses sozialer Etikettierung bzw. einem psychiatrischen Verständnis von Behinderung als soziale Konstruktion verbunden (Cloerkes 2007: 10f). 
derer Gerechtigkeit verstanden - in Abgrenzung von der generellen Tugend der Gerechtigkeit als eine Form des Tun und Handelns (Aristoteles NE 1130 b 30ff., Gigon 2004: 208).

Schramme zufolge erfolgt diese Gleichsetzung wesentlich seit John Stuart Mill (Schramme 2006: 23). Zeitgenössische Ansätze, die sich mit der Verteilung von Gütern und Lasten sozialen Zusammenlebens beschäftigen, haben dann vor allem seit Rawls eine Renaissance erfahren (Ladwig 2004: 120). Bei Mill sollen sich alle gesellschaftlichen Institutionen und aufrechten Bürger am obersten Prinzip sozialer oder austeilender Gerechtigkeit ausrichten: Jeder soll gleich behandelt werden, der sich in gleichem Maße verdient gemacht hat (Mill im Aufsatz „Utilitarianism“ in „On Liberty and other Essay“, nach Schramme 2006: 23). Dieses Verständnis beruht wiederum auf einem aristotelischen Grundverständnis, das modern als „formales Gebot der Willkürfreiheit“ (Ladwig 2011a: 20, vgl. Tugendhat 1993: 373) der Gerechtigkeit gedeutet wird: Gleiches soll gleich behandelt werden (Aristoteles NE 1131 a 20ff., Gigon 2004: 209). Das impliziert z.B. den Ausschluss der Regelung von Konflikten durch Kräfteverhältnisse - das Recht des Stärkeren ist nicht tatsächlich ein Recht (so Rousseau, nach Ladwig 2011a: 31), denn er kann sich aufgrund seiner Dominanz willkürlich nehmen, was er will.

Gerechtigkeit ist in diesem Sinne etwas normativ Vorzugswürdiges: eine willkürfreie Entscheidung zwischen konfligierenden gültigen Ansprüchen. Jeder bekommt, was ihm „zusteht“ (ebd.: 22f., 6f.). Diese „gültigen Ansprüche“ müssen spezifiziert werden im Hinblick auf bestimmte Regeln (ebd.).

Verteilungstheorien der Gerechtigkeit müssen mindestens eine Metrik - bzw. was Gerald Cohen als „Währung“ von Gerechtigkeitstheorien bezeichnet hat (1989) - und eine Verteilungsregel spezifizieren (Anderson 2010: 81). Die Metrik bestimmt den Typus von Gut, das in der Beurteilung gerechter Verteilungen zugrunde liegt. Welches die akzeptabelste Metrik sei, wurde in der so genannten Equality of What-Debatte kontrovers diskutiert. In den hier berücksichtigten zentralen Ansätzen sind die Metriken folgende: Grundgüter (Rawls), Ressourcen (Dworkin), Welfare oder ,Wohlergehen' (Arneson und begrenzt Cohen) und Capabilities (Sen, Nussbaum, Anderson). Solche Metriken können subjektiv oder objektiv sein. Subjektive Metriken bestimmen die Besserstellung von Individuen auf der Basis von deren Vorlieben und Interessen, objektive Metriken sind unabhängig von einer solch internen Perspektive (Scanlon 1975: 656, 658). Ich beschränke mich für die Zwecke der Arbeit auf objektive Metriken, u.a. weil diese verständlicher die Dringlichkeit von gerechtigkeitsbasierten moralischen Ansprüchen begründen können (ausführlich: ebd., speziell 659ff.) und weil sie zumindest schlüssiger das von Rawls formulierte ,public criterion of justice“ (u.a. Rawls 2003: 5) erfüllen können - ihre Prinzipien können in öffentlichen Diskursen hervorgebracht, affirmiert und internalisiert werden (vgl. Anderson 2010: 81). Es wird daher z.B. keine utilitaristische Metrik berücksichtigt. 
Hinsichtlich der Verteilungsregeln unterscheidet Anderson unter anderem zwischen solchen, die ,Muster' (,patterns “) der Verteilung berücksichtigen und jenen, die das nicht tun. Zu ersteren zählt sie etwa Maximierungsregeln, wie sie oft in utilitaristischen Metriken zur Anwendung kommen, zu letzteren streng egalitäre, prioritäre und Suffizienzregeln der Verteilung (ebd.: 82). Angesichts der berücksichtigten Literatur wird sich die Auseinandersetzung auf die letzten drei Varianten beschränken.

Hängt Gerechtigkeit begrifflich mit einer wie auch immer charakterisierten Form von Gleichheit zusammen? Ich möchte mich nicht zuletzt im Hinblick auf den hier favorisierten CapabilityAnsatz „Demokratischer Gleichheit“ Bernd Ladwig darin anschließen, dass Gleichheit zumindest nicht schon zur „Grammatik“ des Gerechtigkeitsdenkens gehört (Ladwig 2011a: 81). Gerechtigkeit ist begriffsgeschichtlich lange anders gedeutet worden und Widersprüche kehren in der so genannten Why-Equality-Debatte zeitgenössisch wieder (zur Übersicht die Beiträge in Krebs 2000, beteiligt sind unter anderem Anderson und Parfit). Da diese weitreichende Kontroverse jedoch für eine angemessene Behandlung ebenfalls mehr Platz einfordern würde, als hier zur Verfügung steht, werde ich mich im Sinne von Martha Nussbaums Position in dieser Frage jenseits einer Stufe der Suffizienz agnostisch verhalten.

Schließlich sei angemerkt, dass die Inklusion von Menschen mit Schädigungen in Theorien der Gerechtigkeit keinen moralischen Automatismus darstellt. Es ist unter anderem das Verdienst von Allen Buchanan, der ein Kapitel zur „Moralität der Inklusion“ im Band From Chance to Choice (2000) verfasst hat, darauf hingewiesen zu haben, dass das Interesse an Inklusion von Menschen mit Schädigungen nicht unumstritten ist: Es steht zumindest potenziell in Spannung zu einem „maximizing interest“": ,,an important and morally legitimate interest in access to the most productive and rewarding cooperative scheme available“ (Buchanan et al. 2000: 290ff.). Wie in Abschnitt 2.1 erwähnt wurde, ist Behinderung nicht zuletzt sozioökonomisch ein signifikanter Kostenfaktor. Ein wesentlicher Aspekt politischer Gerechtigkeit ist es schließlich auch, widersprechende Interessen vor dem Hintergrund knapper Ressourcen zu berücksichtigen. Buchanan ist jedoch letzten Endes der Meinung, dass die Inklusion von Menschen mit Schädigungen moralische Priorität genießt:

„Since exclusion from the dominant cooperative framework is damaging in many ways, [theories of
subject-centered justice] require that we manifest our equal respect for all by undertaking efforts to ena-
ble individuals to be full participants in the dominant cooperative scheme” (Buchanan et al. 2000: 295).

Theorien subjektzentrierter Gerechtigkeit sind für Buchanan all jene, die im Gegensatz zu Theorien auf Eigeninteresse beruhender Reziprozität (z.B. die Ansätze von Thomas Hobbes und David Gauthier) die Mitgliedschaft in der Rechtsgemeinschaft nicht von einem Nettobeitrag zum System sozialer Kooperation abhängig machen - effektiv also alle hier berücksichtigten Ansätze. Thomas Schramme spricht für den Bereich politischer Inklusion von einer ,,minimalistischen Forderung der 
Gerechtigkeit“ (2006: 222f.). Die Ermächtigung an politischen Entscheidungsprozessen mitzuwirken befähige Menschen erst an Kämpfen um Gerechtigkeit teilzunehmen (ebd.).

\subsection{Rawls}

Es kann in den folgenden Abschnitten nicht darum gehen, zu argumentieren, dass ein alternativer gerechtigkeitstheoretischer Ansatz Rawls in allen Belangen überlegen ist. Wie bereits erwähnt, gibt es auch Ansätze, die versuchen Rawls Grundgütermetrik dahingehend anzupassen, dass sie das Problem des Phänomens Behinderung inkludieren kann ${ }^{13}$. Darüber hinaus , borgen’ sich in der Metrik und Verteilungsregel von Rawls differierende Ansätze Aspekte von Rawls Argumentation - Martha Nussbaum besonders deutlich z.B. die Idee des „overlapping consensus “ (,übergreifender Konsens“) und auch die Vorstellung eines „Politischen Liberalismus“, in dem Menschen mit unterschiedlichen umfassenden Vorstellungen des Guten dennoch hinsichtlich eines Kerns gemeinsamer Werte - in Rawls Fall die Prinzipien der Gerechtigkeit - übereinkommen können (Nussbaum 2007: 6, 163, Rawls 2003: 32ff., 40f.). Es soll aber deutlich gemacht werden, dass Rawls vor allem an der Inklusion von Menschen mit schweren Schädigungen aufgrund verschiedener konstitutiver Aspekte seiner Konzeption scheitert.

\subsubsection{Gerechtigkeit als Fairness}

Rawls erhebt mit seinem vertragstheoretischen Ansatz der Gerechtigkeit als Fairness im Gegensatz zu den anderen im Anschluss vorgestellten Konzeptionen den Anspruch einer umfassenden Theorie politischer Gerechtigkeit - wenn auch nicht einer kompletten Moraltheorie (2003: 12). Gleichzeitig bezweifelt Rawls selbst, insbesondere in den Modifikationen seit A Theory of Justice, dass das Thema Behinderung in seinem Ansatz adäquat berücksichtigt werden kann. Hinsichtlich der Frage, was jenen Individuen geschuldet ist, die den freien und gleichen Personen eigenen so genannten „moralischen Vermögen“ (siehe weiter unten) entweder temporär, durch Krankheit und Unfall, oder permanent nicht aufweisen, befürchtet Rawls, dass dies eins der Probleme sein könnte ,[...] on which justice as fairness may fail [...]“ (2005: 21).

Es sollen in diesem Abschnitt zentrale Aspekte von Rawls Ansatz auf die Frage der Berücksichtigung von Behinderung zugespitzt werden. Dazu beziehe ich mich vornehmlich auf Justice as Fairness - A Restatement (2003), Rawls chronologisch letzte systematisch überarbeitete Variante seiner

13 Diese Versuche, wie sie prominent etwa von Norman Daniels, Erin Kelly und Thomas Pogge unternommen werden (siehe z.B. die jeweiligen Beiträge in Brighouse / Robeyns 2010), müssen angesichts Rawls eigener expliziter Aussagen (s.u.) aber zumindest immer ein Stück weit „Rawls gegen Rawls“ interpretieren. 
Überlegungen aus $A$ Theory of Justice. Nur ergänzend, oder in Fällen, in denen Rawls' Position sich in für das Thema relevanten Hinsichten signifikant verändert hat, verweise ich auch auf $A$ Theory of Justice und Political Liberalism. Rawls hat einige dieser Änderungen zuerst in eigenständigen Artikeln formuliert, ich werde mich im Anschluss aber der Einfachheit halber auf Unterschiede zwischen den berücksichtigten Monographien beziehen.

Wie bereits in der Einleitung erwähnt, nimmt Rawls Konzeption Ausgang von folgender idealisierten Grundfrage nach Gerechtigkeit: „What is the most acceptable political conception of justice for specifying the fair terms of cooperation between citizens regarded as free and equal and as both reasonable and rational, and (we add) as normal and fully cooperating members of society over a complete life, from one generation to the next?" (2003: 7f.). Ausgehend von dieser Fragestellung möchte ich nun drei problematische Bereiche für die gerechtigkeitstheoretische Inklusion des Phänomens Behinderung herausstellen.

\subsubsection{Die Gesellschaft als ein System fairer Kooperation: Öffentlichkeit, Reziprozität und rationale Vorteile}

In Abgrenzung zum Verständnis einer demokratischen Gesellschaft als „Gemeinschaft“ (die eine einzige umfassende, also vermutlich repressive Doktrin des umfassend Guten aufweist), oder „Assoziation“ (in die man jederzeit nach Belieben ein- und austreten könnte) skizziert Rawls erstere als ein „System fairer Kooperation“ (2003: 3f.). Die gewonnenen Prinzipien politischer Gerechtigkeit sollen dann in diesem System sicherstellen, dass Bürger effektiv frei und gleich sind.

Das Verständnis der Gesellschaft als System fairer Kooperation weist laut Rawls drei zentrale Eigenschaften auf: Erstens geht es im Sinne der oben benannten Affirmation um öffentlich anerkannte Regeln und Verfahren, die von den im System Partizipierenden als angemessen anerkannt werden - nicht einfach um sozial koordinierte Handlungen. Zweitens können und sollten die Bedingungen fairer Kooperation durch jeden akzeptiert werden, vorausgesetzt, dass auch andere sie akzeptieren. Drittens schließlich hänge die Idee der Kooperation mit der Erwartung eines Guts oder rationalen Vorteils zusammen. Diesen rationalen Vorteil würden die Kooperierenden vom Standpunkt ihrer eigenen Vorstellungen des Guten aus verfolgen (ebd.: 6).

Die Bedingungen der Kooperation beruhen somit auf einem Verständnis von Gegenseitigkeit oder Reziprozität, das auch erfordert, dass alle in angemessener Weise von der Kooperation profitieren - eine grundlegende Idee des Gesellschaftsvertrags. Das Verständnis von Reziprozität findet sich auch in einem der zwei Gerechtigkeitsprinzipien wieder, zu denen Rawls letztendlich gelangt (dem Differenzprinzip) und es ist ein Faktor, der zur Stabilität einer wohlgeordneten Gesellschaft beitragen soll. 
Gleichzeitig meint Rawls in Political Liberalism, Reziprozität sei explizit nicht mit gegenseitigem Nutzen gleichzusetzen, denn besonders diejenigen, die viel Eigentum oder ein großes Vermögen haben, werden durch die Kooperation in absoluter Hinsicht nicht unbedingt besser gestellt - sie könnten sogar signifikant schlechter gestellt werden (ebd.: 17). Martha Nussbaum kommentiert, in Political Liberalism sei die Reziprozität damit eher als Eigenschaft der idealisierten ,wohlgeordneten Gesellschaft“ zu verstehen, die Vorstellung des gegenseitigen Vorteils bleibe aber im Hinblick auf die Zustimmung zu den Gerechtigkeitsprinzipien erhalten (Nussbaum 2007: 60ff.). Die Eigenschaften des Systems fairer Kooperation stellen ein erstes potenzielles Problem für die gerechtigkeitstheoretische Inklusion von Menschen mit Schädigungen dar, wie im Anschluss argumentiert werden soll.

\subsubsection{Freie und gleiche Bürger: Über eine Lebensspanne voll kooperierende Personen}

Die Charakterisierung von Personen als frei und gleich ist urliberal, sie findet sich unter anderem im Naturzustand bei John Locke wieder. Sie ist, wie Rawls selbst anerkennt, im Hinblick auf die soziale Realität eine nicht unwesentliche Idealisierung (Rawls 2003: 7), als Ziel aber „realistisch utopisch“, wie Rawls meint (ebd.: 4). Wie in der Einleitung bereits angeführt, hält Rawls diese idealisierenden abstrakten Konzepte für die Frage nach den Prinzipien der Gerechtigkeit einer Gesellschaft als System fairer Kooperation für nötig: ,[T] hey are used to gain a clear and uncluttered view of a question seen as fundamental by focusing on the more significant elements that we think are most relevant in determining its most appropriate answer" (ebd.: 8).

Es handelt sich explizit um eine normative Konzeption, so Rawls, die nicht mit einer deskriptiven Konzeption des Menschen, z.B. als Angehöriger der Spezies homo sapiens, zu verwechseln sei. Sie sei stattdessen auch philosophisch-historisch als Voraussetzung für die Teilnahme am sozialen Leben gedeutet worden, und ist hier die Voraussetzung für die Rolle als ,,voll kooperierendes Mitglied über eine volle Lebensspanne“، im System fairer Kooperation (ebd.: 24).

Seit Political Liberalism ist das normative Verständnis von Personen als frei und gleich verbunden mit zwei „,moralischen Vermögen“ („two moral powers“, Rawls 2005: 19). Es handelt sich dabei um die Fähigkeiten, einen Gerechtigkeitssinn und eine Konzeption des Guten zu entwickeln (ebd.: 18f., 2005: 19). Während in A Theory of Justice der moralische Personenstatus noch als „Potenzialität" galt, die normalerweise von beinahe jedem entwickelt wird und als Grundlage voller Autonomie galt (Rawls 1971: 505), sind spätestens seit Political Liberalism explizit nur Personen mit diesen Vermögen in der Lage, an einem reziproken System gegenseitiger Vorteile teilzunehmen, und die fairen Bedingungen und Regeln aus Einsicht zu befolgen (Rawls 2003: 19).

Auch die an dieser Stelle aufgrund des systematischen Zusammenhangs vorgezogene Formel „,normal and fully cooperating members of society over a complete life” (Rawls 2003: 7f.) ist eine 
Neuerung. In A Theory of Justice war die Schwelle zum Gerechtigkeitssubjekt noch recht niedrig: der moralische Personenstatus sollte zwar eine hinreichende Bedingung für die Inklusion sein, nicht aber unbedingt eine notwendige - diese Frage ließ Rawls offen (Rawls 1971: 506, vgl. Graumann 2011: 217f.). Weil dieser Status in Theory auf einer ,Kapazität“ (,capacity“) und nicht der faktischen Präsenz (,realization“) der (dort noch nicht so genannten) zwei moralischen Vermögen beruhen sollte, ging Rawls davon aus, dass er weder für Kinder noch für Menschen, die diese Kapazität auch permanent nicht aufweisen, ein folgenreiches Problem darstellen würde (ebd.: 509f.). Die Frage marginaler Fälle, die diese Bedingung nicht erfüllen, sei daher kein ernsthaftes praktisches Problem. Rawls fügte hinzu, dass selbst wenn der moralische Personenstatus eine notwendige Bedingung wäre, es dennoch praktisch unklug sei, Gerechtigkeit aus diesen Gründen in den betroffenen Fällen vorzuenthalten. Auch wenn Individuen z.B. unterschiedliche Fähigkeiten für die Ausbildung eines Gerechtigkeitssinns hätten, sollten sie dennoch dem vollen Schutz der Gerechtigkeitsprinzipien unterliegen (ebd.).

Seit Political Liberalism sollen nun aber die zwei moralischen Vermögen und zusätzlich die Qualifizierung als normaler und voll kooperierender Teilnehmer über eine komplette Lebensspanne ausschlaggebend für die Inklusion sein. Rawls führt an, dass diese Ergänzung eine Bedingung sei, um die Personen im Urzustand hinreichend gleich zu machen und so unempfindlicher gegenüber willkürlichen Kräfteverhältnissen (2005: 19). Diese Kennzeichnungen sind eine zweite erwähnenswerte substanzielle Modifikation der Rawls'schen Position seit A Theory of Justice und auch ein zweiter potenzieller Problembereich für die Inklusion von Menschen mit Schädigungen.

\subsubsection{Die akzeptabelste Konzeption: Zwei Prinzipien der Gerechtigkeit, die Metrik der Grundgüter und der Index nach Reichtum und Einkommen}

Was ist also Rawls zufolge die akzeptabelste Konzeption politischer Gerechtigkeit unter diesen Bedingungen? Rawls beschreibt in der Tradition klassischer Vertragstheorien zur Veranschaulichung einen fiktiven Urzustand (,original position“) mit einer Wahlsituation, in der Prinzipien einer gerechten Gesellschaft bestimmt werden sollen, denen jeder zustimmen kann. Entgegen manchen Interpretationen macht Rawls jedoch zunehmend deutlich, dass die Rechtfertigung des Ansatzes nicht auf diesem Gedankenexperiment lastet. Diese erfolgt über das so genannte „Überlegungsgleichgewicht“ (,reflective equilibrium“) (2003: 26, ausführlicher 2005: 381ff.). Rawls meint, kurz gefasst, dass im Sinne eines „Kohärenzverfahrens“ die Gerechtigkeitsprinzipien mit unseren alläglichen moralischen Überzeugungen über einen Prozess der Rekonstruktion in Übereinstimmung gebracht werden können (vgl. Ladwig 2011a: 142f., Horn / Scarano 2002: 339, ausführlich auch Schramme 2006: 67ff.).

Das Wissen der Personen im Urzustand ist durch einen so genannten „Schleier des Nichtwissens“ (,veil of ignorance“) eingeschränkt. Die Personen im Urzustand, die als Vertreter späterer tatsächlicher Bürger gelten sollen, haben hinter diesem Schleier zwar ein generelles Wissen über die mensch- 
liche Gesellschaft - wie z.B. grundlegende menschliche Bedürfnisse und ökonomische Prinzipien einer Gesellschaft. Sie wissen aber nicht, welche tatsächlichen sozialen Positionen die Menschen, die sie vertreten (seit Political Liberalism), einnehmen werden - d.h. zum Beispiel welches Einkommen, welche Hautfarbe, welches Geschlecht und auch welche natürlichen Fähigkeiten und Talente sie aufweisen werden (Rawls 1971: 136ff.).

Die Parteien in einem so charakterisierten Urzustand würden laut Rawls letzter Fassung zu zwei Prinzipien gelangen, einem Prinzip „,völlig adäquater Rechte“ und zum Differenzprinzip, die auf die Grundstruktur einer Gesellschaft, also die grundlegenden politischen Institutionen wie die Verfassung und die wirtschaftliche und sozialstaatliche Organisationsform, angewendet werden sollen. Diese zwei Prinzipien stellen Rawls Auffassung nach „demokratische Gleichheit“ her (z.B. 1971: 75):

1. Each person has the same indefeasible claim to a fully adequate scheme of equal basic liberties, which scheme is compatible with the same scheme of liberties for all; and

2. Social and economic inequalities are to satisfy two conditions: first, they are to be attached to offices and positions open to all under conditions of fair equality of opportunity; and second, they are to be to the greatest benefit of the least-advantaged members of society (the difference principle)" (Rawls 2003: 42).

Wie werden die „am wenigsten begünstigten Angehörigen“ identifiziert? Es sind diejenigen, die im Hinblick auf die Metrik der Grundgüter am schlechtesten gestellt sind (Rawls 2003: 57f.). Rawls unterscheidet fünf Arten von Grundgütern: Die Grundfreiheiten, Freizügigkeit und freie Berufswahl, mit verantwortungsvollen Ämtern verbundene Befugnisse und Vorrechte, Einkommen und Besitz als allgemein dienliche Mittel und die sozialen Grundlagen der Selbstachtung (ebd.: 58f.). Diese sind so genannte ,, all-purpose goods “, von denen jeder lieber mehr als weniger haben will, und die wertvoll sind, egal welche umfassende Vorstellung des Guten die Bürger jeweils verfolgen wollen.

Die Grundgüter müssen jedoch zum Zweck der Vergleichbarkeit verschiedener Positionen auf einen angemessenen Index hin spezifiziert werden (ebd.: 59). In einer wohlgeordneten Gesellschaft, in der allen Bürgern gleiche Rechte und eine faire Chancengleichheit zugesichert sind, fallen die am wenigsten Begünstigten schließlich in die Einkommensklasse ,mit den geringsten Erwartungen“: ,$[\ldots]$ in its simplest form the least advantaged are not identifiable apart from income and wealth - they are not individuals identified by natural or other features“"(ebd., Fn. 26). Dies ist ein drittes potenzielles Problem für die gerechtigkeitstheoretische Inklusion des Phänomens Behinderung. 


\subsection{Die Kritik an Rawls im Hinblick auf die Inklusion von Menschen mit Schädigungen}

Dem Thema der Arbeit angemessen werde ich mich in der Rawls-Kritik auf die drei im letzten Abschnitt identifizierten problematischen Faktoren für die Inklusion von Menschen mit Schädigungen beschränken, vor allem im Hinblick auf die interaktionistischen Grundzüge eines angemessenen Modells (ohne schon zu viel hinsichtlich der alternativen Metriken vorwegzunehmen).

Ich beziehe mich dafür unter anderem auf die Kritik der US-amerikanischen Philosophin und Capability-Theoretikerin Martha Nussbaum aus Frontiers of Justice (2007). Ähnliche Einwände sind zum Teil schon früher von anderen Theoretikern erhoben worden, wie etwa von Amartya Sen (1980) und Ernst Tugendhat (1998), und auch von feministischen Kritikerinnen wie z.B. Eva Feder Kittay und Anita Silvers, auf die Nussbaum sich bezieht.

Einer der zentralen Kritikpunkte aus Frontiers of Justice ist bereits für die ersten beiden Faktoren relevant: Rawls schließe aufgrund der vertragstheoretischen Struktur seines Arguments - ähnlich wie andere Theoretiker der Vertragstradition - die Frage danach, von wem die Prinzipien der Gerechtigkeit gewählt werden mit der Frage, für wen sie gewählt werden kurz (Nussbaum 2007: 16). Nur diejenigen, die die Anforderungen des moralisierten Personenbegriffs erfüllen und normal kooperierende Mitglieder eines System fairer Kooperation sein können, sind auch Subjekte grundlegender Gerechtigkeit.

Rawls Ansatz hat vor allem Schwierigkeiten mit schweren Formen von Schädigungen, je nach Aspekt mit körperlichen, geistig-psychischen oder beiden Formen. Die Wichtigkeit solcher ,extreme cases“, wie Rawls meint, solle nicht bestritten werden (2003: 176). Auch Menschen mit Schädigungen seien bestimmte Pflichten geschuldet - unabhängig davon wie schwer ihre Schädigungen seien (ebd.). Es sei jedoch fraglich, welches Gewicht diese Pflichten gegenüber anderen grundlegenderen Ansprüchen hätten. Es kann sich hier folglich nur um imperfekte moralische Pflichten handeln, mit denen keine starken Ansprüche korrelieren - wie auch der Rawls-Schüler Thomas Pogge konstatiert (Pogge 2010: 52f.). Nussbaum meint, dass es sich letztendlich nur um moralische Motive der Barmherzigkeit und Nächstenliebe drehen könne (Nussbaum 2007: 118). Menschen mit Schädigungen sind so auf die Wohltätigkeit und Pflichterfüllung ihrer Mitbürger angewiesen, anstatt notfalls einklagbare und durchsetzbare Rechte zur Verfügung zu haben (vgl. Felder 2012: 35).

\subsubsection{Reziprozität, rationale Vorteile und Behinderung}

Die Ideen der Reziprozität und der rationalen Vorteile der Kooperation sind wie erwähnt klassische vertragstheoretische Motive: In Hobbes Leviathan etwa werden in Erwartung der Vorteile der Koope- 
ration die natürlichen Freiheiten des vorvertraglichen Zustands aufgegeben, und die Bürger unterwerfen sich Gesetzen unter der Voraussetzung, dass diese alle gleich binden.

Rawls zufolge sind es die Gerechtigkeitsprinzipien - vor allem das Differenzprinzip - die der Vorstellung der Reziprozität Ausdruck verleihen (2005: 17). Dahinter verbirgt sich die Idee, dass Menschen ihre natürlichen Talente nicht im moralischen Sinne verdienen. Eine gerechte Gesellschaft belohnt die Aus- und Fortbildung solcher Talente zwar, etwa indem die fraglichen Personen im Sinne fairer Chancengleichheit begehrte Ämter und Positionen einnehmen können - dies ist aber an die Bedingung geknüpft, dass die am wenigsten Begünstigten von solch einem Arrangement am meisten profitieren (2003: 74ff, 124). Die in der ,Begabungslotterie' Glücklichen haben somit den Vorteil begehrenswerter Positionen mit vermutlich höheren Verdiensten - die nach Einkommen und Vermögen am schlechtesten Gestellten haben hingegen den Vorteil eines Transfers, z.B. in Form von progressiven Steuern auf die höheren Einkommen.

Dies ist ein Zusammenhang, der z.B. von den durch Elizabeth Anderson so benannten ,Glücksegalitaristen' - z.B. Arneson, Dworkin und Cohen grundlegend anders interpretiert worden ist, wie ich in Abschnitt 3.3 darstellen will. Die Bürger in einer gerechten Gesellschaft werden bei Rawls nicht aufgrund von individuellen Variationen bezüglich natürlicher Begabungen oder eben Schädigungen kompensiert: Der Einfluss der moralisch arbiträren Verteilung natürlicher ,Ausstattungen’ soll in einer Gesellschaft, deren Institutionen durch die zwei Gerechtigkeitsprinzipien reguliert werden, effektiv aufgehoben, nicht entschädigt werden (Scheffler 2003: 25, vgl. Anderson 1999a: 290). Die Gruppe der am wenigsten Begünstigten, die laut Differenzprinzip am meisten von ökonomischen und sozialen Ungleichheiten profitieren müssen, sind nicht nach Merkmalen natürlicher Eigenschaften definiert, sondern letzten Endes nach Reichtum und Einkommen (Rawls 2003: 59, Fn. 26; vgl. Abschnitt 3.2.3).

Zumindest im Hinblick auf schwere körperliche und geistig-psychische Schädigungen ist es aber fraglich, ob betroffene Personen die Anforderungen eines so charakterisierten Systems reziproker Erwartung von Vorteilen erfüllen können, bzw. ob sie es aus Einsicht befolgen können. Für Rawls ist die Antwort klar: Es werden nur die Personen innerhalb einer ,normalen Bandbreite“ von Fähigkeiten (ebd.: 171) berücksichtigt - diejenigen, die solch schwere Beeinträchtigungen haben, dass sie keinen solchen normalen Beitrag erbringen können, werden nicht berücksichtigt und sind somit auch keine Träger von auf Gerechtigkeit basierenden Ansprüchen (ebd.: 170, vgl. Nussbaum 2007: 105).

Im Hinblick auf das Thema der Inklusion des Phänomens Behinderung wäre es hingegen wünschenswert, eine weniger anspruchsvolle Fassung von Reziprozität zu entwickeln (vgl. Anderson 1999a: 326). Diese sollte z.B. die Vielfalt von Schädigungen berücksichtigen können (siehe Abschnitt 2.2) - so auch Nussbaum (2007: 92) - und die vielfältigen Interaktionen mit Kontextfaktoren. In vielen Fällen von Behinderung ist die Frage, zu welchem Ausmaß ein Mensch produktiv sein oder 
einen Beitrag zum Gemeinwesen leisten kann, entscheidend abhängig von der Art und dem Ausmaß der Förderung, die zur Verfügung steht (Nussbaum 2007: 190). Fehlende Förderung kann ein angemessenes Verständnis des Potenzials vieler Menschen mit Schädigung auch auf Wohlergehen und ein selbstbestimmtes Leben verhindern.

Ein weiter gefasstes bzw. inklusiveres Verständnis von Reziprozität, wie es z.B. Lawrence Becker in seinem Artikel „Reciprocity, Justice and Disability“ (2005) und Nussbaum in Frontiers of Justice entwickelt, könnte Abhilfe schaffen. Becker kritisiert unter anderem, dass Rawls Verständnis der Reziprozität eine weit verbreitete vereinfachte Vorstellung eines „,fairen Gegenwerts“ repräsentiere (ebd.: 20). Reziprozität, z.B. als gleicher Grenzaufwand („equal marginal sacrifice“), könne hingegen einen weiteren Rahmen proportionaler und angemessener Gegenleistungen abstecken (ebd.: 31 ). Nussbaum entwickelt mit ihrer ,Soziabilität“ ein ähnliches Verständnis, das sowohl symmetrische als auch asymmetrische Beziehungen berücksichtigen soll (2007: 160). Wie die Dinge stehen, herrscht bei Rawls aber letzten Endes nur zwischen „gleichen“ bzw. in relevanten Hinsichten hinreichend ähnlichen Menschen, die ,normal viel' zum System fairer Kooperation beitragen, Reziprozität (Rawls 2003: 170) - und das verhindert potenziell die Inklusion vieler Menschen mit schweren Schädigungen, die so keine Gerechtigkeitsansprüche haben.

\subsubsection{Normal kooperierende Personen mit zwei moralischen Vermögen}

Die moralische Konzeption der Person ist bei Rawls ebenso wie der Status als „,normal Kooperierender" die Grundlage für die gerechtigkeitstheoretische Inklusion. Es handelt sich um die Eigenschaften, die Menschen zu Subjekten von Gerechtigkeit als Fairness machen: „I now turn to the basis of equality, the features of human beings in virtue of which they are to be treated in accordance with the principles of justice“ (Rawls 1971: 504, vgl. 2003: 20). Die zwei moralischen Vermögen sollen unter anderem sicherstellen, dass die Personen die Gerechtigkeitsprinzipien verstehen und aus Einsicht befolgen können.

Die Gleichheit der Personen ist ein Aspekt der größtenteils von David Hume aus „, A Treatise of Human Nature“ übernommenen „Umstände der Gerechtigkeit“, in denen es Rawls zufolge sowohl möglich als auch nötig sei zu kooperieren (Rawls 1971: 126ff.). Diese können in objektive und subjektive Umstände unterteilt werden: Zu ersteren gehören z.B. die Koexistenz mehrerer Individuen an einem Ort und zu einer Zeit, die ungefähre Gleichheit derselben hinsichtlich physischer und geistigpsychischer Kräfte, sowie moderat knappe Ressourcen (Nussbaum 2007: 27). Zu den subjektiven Umständen zählen hingegen ungefähr gleiche oder komplementäre Bedürfnisse und Interessen, bei gleichzeitig unterschiedlichen Lebensplänen. Nötig wird eine Übereinkunft also unter anderem wegen knapper Ressourcen, möglich weil die Bedürfnisse hinreichend ähnlich sind. 
Darüber hinaus soll die ungefähre Gleichheit auch die Unparteilichkeit der Prinzipien ermöglichen bzw. der bildhaften Parteien im Urzustand: Einzelne Gruppen könnten sonst andere einfach aufgrund größerer körperlicher Kraft oder größerer Klugheit und einem Mehr an Verhandlungsgeschick über den Tisch ziehen, bzw. in der Wahl und Auslegung der Gerechtigkeitsprinzipien dominieren.

Die Freiheit der Personen soll unter anderem sicherstellen, dass niemand von anderen besessen wird oder extrem abhängig ist, sondern sich fremder Herrschaft nur auf der Grundlage eigener $\mathrm{Zu}-$ stimmung unterwirft, und dass verschiedene Individuen frei sind, unterschiedliche Konzeptionen des Guten zu entwickeln und zu verfolgen (vgl. Nussbaum 2007: 28f.). Dennoch erinnert diese Auffassung ,,verdächtig an die liberale Wertschätzung einer autonomen Lebensführung“, so Ladwig (2011a: 175), die Rawls im Sinn des overlapping conensus vermeiden will und muss.

Beide Charakterisierungen müssen wiederum in Hinsicht auf Menschen mit schweren körperlichen und geistig-psychischen Schädigungen als problematisch gelten. Weder haben Menschen mit schweren körperlichen Schädigungen in der Regel gleiche physische Kräfte und Menschen mit schweren geistig-psychischen Schädigungen gleiche geistige Kräfte, noch sind vermutlich grundlegende Bedürfnisse und Interessen gleich. Ein bereits genanntes Beispiel sind spezielle Bedürfnisse wie teure Medizin oder Hilfsmittel wie Rollstühle. Diese Bedürfnisse kommen so nicht in den Blick gerechtigkeitstheoretischer Überlegungen.

Was die Freiheit anbelangt (wie letztendlich auch Phasen begrenzter physischer und geistiger Kräfte), ist von verschiedenen Autoren betont worden, dass Abhängigkeit als universelle menschliche Eigenschaft verstanden werden sollte (so z.B. Eva Kittay in Love's Labour, Alasdair MacIntyre in Dependent Rational Animals). Wenn man, wie in Abschnitt 2.1 und 2.7 skizziert, die Kategorie Behinderung als Teil eines Kontinuums menschlicher Zustände - über eine Lebenszeit - versteht, wird augenscheinlich, dass dies auch auf Abhängigkeit zutrifft. Jeder Mensch durchläuft als Kind, in Phasen schwerer Krankheit oder nach Unfällen und schließlich im Alter Abschnitte unvollständiger Freiheit und Autonomie. Die Bedingung der Freiheit schließt somit tendenziell all jene aus, die „nicht, noch nicht, oder nicht mehr zu Autonomie fähig sind“ (Graumann 2010: 217).

Daraus sollte noch nicht folgen, dass soziale Angewiesenheit auch normativ grundlegend ist. Autonomie kann dennoch als wünschenswerte Eigenschaft aufgefasst werden. Sigrid Graumann macht z.B. gegen Eva Kittays Version der Care-Ethik und Alasdair MacIntyres Tugend der Abhängigkeit geltend, dass auch die meisten Menschen mit Schädigung Autonomie anstreben, wie es etwa Anhänger des so genannten Independent Living Movement tun - die die De-Institutionalisierung v.a. aus staatlichen Fürsorgeeinrichtungen anstreben (Graumann 2010: 216).

Im Hinblick z.B. auf Selbstverwirklichung und die eigenen Lebenspläne ist Autonomie im Gegensatz zu institutionalisierter Fürsorge durchaus wünschenswert (ebd.). Dennoch sollte vollständige Autonomie keine Voraussetzung für die Inklusion als Gerechtigkeitssubjekt sein. In vielen Modellen 
persönlicher Assistenz oder eines Mentoring für Menschen mit Schädigungen können die Betroffenen im Gegensatz zu staatlicher Vormundschaft in versorgenden Institutionen weitestgehend selbst darüber entscheiden, wie viel und welche Formen der Unterstützung sie benötigen. Dies trifft z.B. auf das staatsfinanzierte schwedische Modell persönlicher Assistenz für Menschen mit kognitiven Schädigungen zu - es ist in der Intensität abgestuft, um jeweils die weitest mögliche Autonomie betroffener Menschen zu bewahren (Nussbaum 2007: 196).

Auch das Vorliegen der zwei moralischen Vermögen ist insbesondere im Kontext geistig-psychischer Schädigungen eine vermutlich in den meisten Fällen zu hohe Hürde. In Rawls Entwurf sind spätestens seit Political Liberalism nicht einmal diejenigen, die nur temporär unter das geforderte Minimum dieser zwei Vermögen fallen, Subjekte grundlegender Gerechtigkeit. Die Behandlung dieser Fälle könne, so Rawls, erst in der ,legislative stage“ erfolgen. Es handelt sich hierbei um die dritte Phase eines vierstufigen Prozesses der Anwendung der Gerechtigkeitsprinzipien, nach der Zustimmung zu den Gerechtigkeitsprinzipien hinter dem Schleier des Nichtwissens und einer verfassungsgebenden Versammlung (Rawls 2003: 48). Erst in dieser Phase sei das Wissen über die ungefähre Häufigkeit verschiedener schwerer Krankheiten und Unfälle verfügbar, das für die Formulierung von Gesundheitspolitiken nötig sei (ebd.: 173).

Zuletzt könnte man Rawls anspruchsvolle normative Konzeption der Person in Hinsicht auf das Überlegungsgleichgewicht auch immanent zu kritisieren versuchen. Rawls formuliert in Justice as Fairness: ,[...] the conception of the person is worked up from the way citizens are regarded in the public political culture of a democratic society, in its basic political texts (constitutions and declarations of human rights) [...]“ (2003: 19). Dem lässt sich entgegnen, dass in vielen Verfassungen liberaler Staaten keine solche anspruchsvollen Kriterien angelegt werden und insbesondere Menschenrechtsabkommen - wie nicht zuletzt das Übereinkommen über die Rechte von Menschen mit Behinderungen (UN 2006) - in der Regel auf sehr viel inklusiveren Voraussetzungen für den Status als Rechtssubjekt beruhen (vgl. etwa Ladwig 2011b: 5). Wie weiter oben gezeigt, sollte jedoch das Überlegungsgleichgewicht als rechtfertigende Grundlage der Gerechtigkeitsprinzipien verstanden werden und nicht der Urzustand mit dem Schleier des Nichtwissens und den dazugehörigen idealisierten Bedingungen. Wenn die resultierenden Gerechtigkeitsprinzipien also z.B. grundlegenden Vorstellungen über die Inklusion von Menschen mit Schädigungen widersprechen, dann sollten die Entscheidungssituation und die dazugehörigen Annahmen verworfen werden, und nicht die moralischen Überzeugungen (vgl. Ladwig 2011a: 181 und Tugendhat 1993: 365). 


\subsubsection{Der vergleichende Index der Grundgüter und die am wenigsten Begünstigten}

Zur Veranschaulichung dieses dritten Problems kann man sich einen Menschen mit Schädigungen vorstellen, der ein genügend hohes Einkommen und Vermögen hat, um in einer Gesellschaft nicht unter die am wenigsten Begünstigten zu fallen - gemessen an Rawls Kriterien. Um alltäglichen Tätigkeiten wie etwa einer Lohnarbeit nachzugehen, benötigt besagter Mensch aber ein besonders teures Medikament oder ein Hilfsmittel wie z.B. einen Rollstuhl. Wenn die Ausgaben für diese besonderen Bedürfnisse vom Vermögen und Gehalt der Person abgezogen werden, gehört sie nun zwar effektiv und ,netto“ zur am schlechtesten gestellten Gruppe - nicht aber der ursprünglichen Position auf dem Index zufolge. Das heißt, die Person hätte keine Ansprüche auf zusätzliche Ressourcen (Ladwig 2011a: 183f.). Selbst wenn Menschen mit Schädigungen nach Abzug solch besonderer Ausgaben nicht schon effektiv zu den am schlechtesten Gestellten gehören, haben sie darüber hinaus dennoch z.B. weniger Ressourcen als andere Menschen übrig, um ihre jeweiligen Lebenspläne zu verfolgen.

Der Grund für den Index ist zum einen wie erwähnt, dass in Justice as Fairness explizit vor allem Reichtum und Einkommen ,, all-purpose goods“ sind, die einen Umtauschwert haben und die von freien und gleichen Personen benötigt werden, um verschiedene Ziele zu verfolgen (Rawls 2003: 58f.). Auf der anderen Seite wären die Grundgüter auf einem vollständigen Index auch kaum kommensurabel: Es wäre z.B. nicht klar, ob ein Mehr eines Grundguts ein Weniger eines anderen aufwiegen kann, und wie verschiedene Personen in einer Rangordnung pluraler Güter positioniert wären. Das ist ein Vorwurf, der Amartya Sen mit seiner Vorstellung gleicher pluraler Capabilites gemacht wird (z.B. Pogge 2010: 51). Wer in einer Gesellschaft effektiv am schlechtesten gestellt ist, hängt aber nicht nur von Reichtum und Einkommen ab, sondern auch von sozialen Umständen und von den unterschiedlichen Fähigkeiten verschiedener Menschen, Ressourcen im Sinne eigener Lebenspläne umzusetzen. Gleichzeitig ist eine Umverteilung zusätzlicher materieller Ressourcen nicht immer die passende Antwort auf verschiedene Formen der Benachteiligung - nicht alle Nachteile sind als Ressourcendefizite verständlich (vgl. Ladwig 2011a: 196).

Sen diagnostiziert diesen Zusammenhang, den er später ,,conversion handicap“ genannt hat (z.B. 2004: 3), zuerst in seinem Artikel „Equality of What“ (1980). Mit der darin unternommenen Rawls-Kritik hat er gleichzeitig entscheidend die nach dem Titel des Artikels benannte Equality-ofwhat-Debatte ausgelöst. In ihr wurde wie erwähnt kontrovers diskutiert, in welcher Hinsicht bzw. entlang welcher Metrik Gleichheit als Angelegenheit von Gerechtigkeit herzustellen sei. Da ich die Capability-Metrik aufgrund ihres besonderen Status für das Thema Behinderung getrennt behandeln will, werde ich auf die Position Sens erst weiter unten eingehen. Zunächst werde ich mich nun mit von Anderson so genannten ,Glücksegalitaristen' auseinandersetzen, die ebenfalls maßgebliche Protagonisten in der erwähnten Debatte waren. 


\subsection{Der ,Glücksegalitarismus'}

Der „Glücksegalitarismus“ („luck egalitarianism“) hat diesen Namen zuerst durch Elizabeth Anderson in ihrem Artikel „, What is the Point of Equality? “ (1999a) erhalten. Der Titel hängt damit zusammen, dass die jeweiligen Vertreter die Überzeugung, dass Lebensaussichten aus Gründen der Gerechtigkeit angeglichen werden sollten, mit einer Unterscheidung zwischen unverantworteten $\mathrm{Zu}$ ständen oder Zufällen (,, brute luck“ oder ,,chance“) und verantwortbaren Zuständen oder Entscheidungen (,, option luck“ oder ,, choice“) verbinden. Die grundlegende Idee dahinter ist, dass Menschen für unverschuldete Einschränkungen der eigenen Lebensaussichten kompensiert werden, für selbst herbeigeführte oder verschuldete Nachteile aber selbst zur Verantwortung gezogen werden sollten. Menschen sind für ihre Lebensaussichten zu einem Teil selbst verantwortlich.

Der Glücksegalitarismus kann aus der Sicht des Themas Behinderung gleichzeitig als eine Antwort auf das bei Rawls diagnostizierte Problem verstanden werden, dass unverdiente Einschränkungen der Lebensaussichten aufgrund ,interner Merkmale', also z.B. Schädigungen, nicht genug berücksichtigt werden. Das Differenzprinzip könne solche Ursachen der Benachteiligung nicht adäquat behandeln. Aus der Tatsache, dass wir unsere natürlichen Einschränkungen nicht moralisch verdienen, sollten Konsequenzen gezogen werden: Nachteile in der ,natürlichen Ausstattung' sollten aus Gründen der Gerechtigkeit kompensiert werden. Arneson, Cohen, Dworkin und letztendlich auch Sen, Nussbaum und Anderson fassen Behinderung daher als einen entscheidenden Grund für das Abweichen von einer Gleichverteilung auf.

Dworkin und Arneson und auch die Capability-Theoretiker können im Gegensatz zu Rawls berücksichtigen, dass eine Behandlung von Menschen mit Schädigungen aufgrund dieser individuellen Zustände eine Ungleichverteilung von Gütern erfordern kann, um Ressourcengleichheit bzw. gleiche Chancen auf Wohlergehen herzustellen. Diese Auffassung findet Ausdruck in Dworkins sprichwörtlicher Unterscheidung zwischen einer Gleichbehandlung (,equal treatment ${ }^{\star}$ ) und einer Behandlung „als Gleicher“ (,,as an equal“) - grundlegend moralisch bedeutsam ist letztere Hinsicht (Dworkin 1981b: 285, 342; Ladwig 2011a: 93).

Der US-amerikanische Philosoph Samuel Scheffler nennt den Glücksegalitarismus in seinem Artikel ,, What is Egalitarianism?“ einen der signifikantesten gerechtigkeitstheoretischen Ansätzte seit Rawls A Theory of Justice (Scheffler 2003: 5). Gleichzeitig sei der Anspruch, diesen Standpunkt aus der Rawls'schen Position herleiten zu können, nicht haltbar (ebd.). Darüber hinaus ist diese ,Familie' von Konzeptionen zu Recht v.a. durch Anderson einer umfangreichen Kritik auch in Hinsicht auf das Thema Behinderung unterzogen worden, wie ich im Anschluss an die Darstellung der Positionen zeigen werde. 


\subsubsection{Dworkin}

Der amerikanische Rechtsphilosoph Ronald Dworkin hat in zwei aufeinander folgenden Aufsätzen in Abgrenzung zu einer Wohlergehensmetrik der Gerechtigkeit eine Konzeption von Ressourcengleichheit entwickelt. Dworkins Ansatz ist aus mehreren Gründen für die gerechtigkeitstheoretische Inklusion von Menschen mit Schädigungen interessant. Insbesondere ist ihm anzurechnen, dass er im Gegensatz zu Rawls die funktionellen und strukturellen Beeinträchtigungen für eine gerechte Verteilung von Gütern berücksichtigt hat.

Im ersten Aufsatz, What is Equality - Part 1: Equality of Welfare (1981a) legt Dworkin ausführlich dar, warum verschiedene Varianten eines Ansatzes gleichen Wohlergehens zurückgewiesen werden sollten. Als größte generelle Herausforderung charakterisiert er das bereits erwähnte Phänomen der teuren Vorlieben bzw. „expensive tastes“ oder „, expensive preferences “, ein in der Gerechtigkeitsphilosophie immer wieder aufgegriffenes Problem (ebd.: 228, vgl. z.B. Arneson 1989: 84, Cohen 1989: 907, Scanlon 1974: 659). Wenn eine Person eine solche teure Vorliebe entwickle, wie etwa nur Champagner trinken zu wollen, dann müsse eine Gerechtigkeitskonzeption der Wohlergehensgleichheit solche Aufwendungen kompensieren. Denn für die Erlangung gleichen Wohlergehens müssten so lange Ressourcen umverteilt werden, bis keine Transfers mehr zu einer weiteren Angleichung des Wohlergehens führen würden. Der Ansatz habe prinzipiell nichts dagegen einzuwenden - was kontraintuitiv sei und unfair erscheine (Dworkin 1981a: 189, 228). Dworkin sieht keine Möglichkeit, das Problem teurer Vorlieben innerhalb dieser Metrik auszuschließen. Versuche, einen Ansatz gleichen Wohlergehens dementsprechend anzupassen, würden letzten Endes zu einer anderen Art von Metrik führen, z.B. zu einer Ressourcenmetrik (ebd.: 240).

Auch die Berücksichtigung von Menschen mit Schädigungen sei innerhalb der Metrik gleichen Wohlergehens nur auf den ersten Blick einleuchtend. Sie liefere zwar einen Erklärungsansatz, warum Menschen mit Schädigungen mehr Ressourcen benötigen könnten als andere - weil sie im Zweifelsfall ungleiche Aussichten auf Wohlergehen gemessen als Präferenzbefriedigung oder Glück hätten. Dies geschehe aber nur zu dem Preis, dass entweder Menschen mit Schädigungen als Gruppe generell niedrigere Aussichten auf Wohlergehen unterstellt würden - was jedoch höchstens eine statistisch zutreffende Annahme sei. Oder jene Menschen mit Schädigungen, die keine Einschränkungen ihres Wohlergehens erleben, würden effektiv aus der Gruppe ausgeschlossen, denen zusätzliche Ressourcen zustehen. Wir seien aus Gründen der Gerechtigkeit aber nicht nur an gleichem Wohlergehen interessiert (ebd.: 241).

Im zweiten Aufsatz Part 2: Equality of Ressources (1981b), stellt Dworkin dem Ansatz gleichen Wohlergehens eine Ressourcenmetrik entgegen. In ihr wird Gerechtigkeit gekennzeichnet als Angelegenheit privater Ressourcen - politische Gerechtigkeit wird zunächst ausgeklammert (ebd.: 283). Dworkin meint, dass marktähnliche Instrumente geeignet seien, um gesellschaftliche Ziele zu iden- 
tifizieren und gleichzeitig individuelle Freiheiten sicherzustellen (ebd.: 284). Er skizziert ein Gedankenexperiment als Modell einer Art von Urzustand, in dem Überlebende eines Schiffsunglücks auf einer unbewohnten Wüsteninsel stranden. Jedem werden als Ausgangspunkt eine bestimmte gleiche Menge Muschelschalen zugeteilt, die in einer Auktion über verschiedene Ressourcen als Zahlungsmittel dienen sollen. Jeder hat prinzipiell ein gleiches Anrecht auf die zu verteilenden Ressourcen, eine gerechte Verteilung soll sich also ausgehend von einer Gleichverteilung einstellen. Jeder kann nun auf verschiedene Ressourcenbündel bieten. Die Aufteilung nach der Auktion soll dann gleich sein, wenn entsprechend einem Neidtest kein Inselbewohner das Ressourcenbündel eines Anderen dem eigenen vorziehen würde (ebd.: 285).

Nachdem eine gewisse Zeit verstrichen ist, könnte der Neidtest jedoch aus verschiedenen Gründen scheitern. Einzelne Menschen könnten aufgrund größeren Glücks oder nützlicher Talente mehr Nutzen aus ihren ursprünglichen Ressourcen schlagen, andere hingegen krank werden und so schlechter gestellt sein (ebd.: 292f.). Um die Relevanz solcher Faktoren für eine gerechte Verteilung zu erörtern, unterscheidet Dworkin an dieser Stelle zwischen so genanntem „bloßem Glück“ (,,brute luck“) und „kalkuliertem Glück“ (,,option luck“) (ebd.: 293). Als Beispiel schlechten „bloßen Glücks“ - bzw. im Deutschen vielleicht eher eines schlechten oder unvorteilhaften Zufalls - könne z.B. angeborene oder, erworbene' Blindheit gelten (ebd.: 296).

Die Auktion soll daher durch einen hypothetischen Versicherungsmarkt ergänzt werden. Eine Versicherung könne in vielen Fällen unvorteilhafte Zufälle in kalkuliertes Glück bzw. kalkulierte Chancen umwandeln, weil die Entscheidung sich gegen solche Zufälle - wie z.B. Behinderung - zu versichern, diese bloßen Zufälle in kalkulierbare Risiken umwandeln könne (ebd.). Unvorteilhafte Zufälle, wie eben physische und geistig-psychische Schädigungen könnten dann Dworkin zufolge in der Regel keine Ansprüche auf Kompensation rechtfertigen - man hätte sich ja gegen sie versichern können (ebd.).

Da Menschen jedoch nur jeweils vor einem Hintergrund tatsächlicher Lebenspläne entscheiden könnten, wie viel sie zu einer solchen Versicherung würden beitragen wollen, schlägt Dworkin alternativ vor, geistig-psychische und physische Fähigkeiten einzelner Personen zu ihren Ressourcen zu zählen (ebd.: 300). Ein in diesen Fähigkeiten eingeschränkt geborener Mensch würde in diesem Sinn mit weniger „,internen“ Ressourcen ausgestattet sein als ein Mensch ohne solche Einschränkungen. Die Konzeption der Ressourcengleichheit würde keinen Vergleich mit einem „,normalen Maß“ an internen Ressourcen implizieren, weil der Versicherungsmarkt bestimmt, für welche Krankheiten und Beeinträchtigungen kompensiert werden müsse (ebd.: 300, Fn.8).

Bei einem Mangel an internen Ressourcen können jedoch Dworkin zufolge nicht einfach interne Ressourcen umverteilt werden, weil diese nicht transferierbar sind (ebd.: 301). Ressourcengleichheit kann solche Ungleichheiten also nicht selbst wett machen, sondern nur mit einem mehr an materiellen 
Ressourcen kompensieren bzw. entschädigen (ebd.: 302). Die Verteilung soll darüber hinaus ,ambition-sensitive“, aber „endowment-insensitive“ sein (ebd.: 311). Das heißt Menschen sollen auf der einen Seite die auf ursprünglich fairen Entscheidungen beruhenden Früchte ihres Bestrebens behalten dürfen, sie sind für diese verantwortlich. Ungleich verteilte Talente müssten jedoch den Neidtest überstehen dürfen. Insbesondere könnten die Arbeitsleistungen strebsamer und talentierter Menschen nicht als eine Art Gemeingut behandelt werden - dies würde zu einer „Sklaverei der Talentierten“ führen (,, slavery of the talented“, ebd.: 312). Auf der anderen Seite sollen sie für Einschränkungen in Ressourcen durch die Ausstattung der natürlichen ,Begabungslotterie' kompensiert werden, denn diese beruhen auf bloßem Zufall. Die unterschiedliche Ausstattung mit Talenten solle also ähnlich der unterschiedlichen Verteilung von Beeinträchtigungen Teil der hypothetischen Versicherung sein und daher ebenso über Steuern als eine Art universelle Versicherungsprämie umgelagert werden (ebd.). Der Unterschied zwischen Talenten und Behinderung sei letztendlich eine Frage des Grades (ebd.: 314).

Diese Konzeption sei angemessener als Rawls Differenzprinzip, das nur unzureichend die Ansprüche derer berücksichtigen könne, die nicht zur Gruppe der am wenigsten Begünstigten, definiert nach Wohlhaben und Einkommen, zählen. Dworkin spezifiziert: „In particular, the structure seems insufficiently sensitive to the position of those with natural handicaps, physical or mental, who do not themselves constitute a worst-off group, because this is defined economically, and would not count as the representative or average member of any such group" (ebd.: 339).

\subsubsection{Arneson}

Der US-amerikanische Philosoph Richard Arneson ist ein Vertreter einer objektiven welfare- bzw. Wohlergehens-Metrik der Gerechtigkeit. In seinem Artikel „Equality and Equal Opportunity for Welfare“ (1989) konzipiert Arneson die Metrik zunächst egalitär - es sollen gleiche Chancen auf Wohlergehen verstanden als Präferenzbefriedigung hergestellt werden. In „Luck Egalitarianism and Prioritarianism “ (2000) schwenkt er auf eine prioritäre Verteilungsregel um.

Der Ansatz folgt der Intuition, dass Grundgüter und Ressourcen eigentlich instrumentell sind für Zustände, um die es Gerechtigkeitstheoretikern eigentlich gehen sollte: das Wohlergehen einzelner Menschen. Arneson sieht darin eine Parallele zum Capabilities-Ansatz: Beide hätten erkannt, dass es nicht um die zu verteilenden Güter selbst geht, sondern darum, wozu diese die Menschen tatsächlich befähigen (1989: 91). Beide Ansätze seien daher eine Antwort auf Sens gegenüber Rawls erhobenen Vorwurf des „Güterfetischismus“ (Arneson 2000: 341, vgl. Sen 1980: 216).

Für den verlässlichsten Wohlergehens-Indikator hält Arneson hypothetische rationale Präferenzen, d.h. solche Präferenzen die ähnlich Rawls ,,considered judgements “ (Rawls 2003: 31) in einem 
ausgeglichenen Zustand unter Einbeziehung aller relevanten Informationen zustande kommen würden. Ähnlich wie Dworkin meint Arneson, dass Ungleichheiten, die aus einer freiwilligen Entscheidung einer Person oder fahrlässigem Verhalten erwachsen, nicht kompensiert werden sollten (1989: 84f.).

Entlang dieser Unterscheidung sei man für manche Präferenzen, die man sinnvollerweise gewählt haben kann, verantwortlich, für andere ,ungewollte“ Präferenzen jedoch nicht. Es müsse etwa im Gegensatz zu Dworkin und Rawls genauer unterschieden werden zwischen Präferenzen, die einfach abänderbar sind und solchen, die Menschen tief eingeschrieben seien oder mit ihrer Identität verbunden sind, und die sie nur zu erheblichen Kosten ändern könnten (ebd.: 82). Die letzteren ,ungewollten“ Präferenzen sollten in der Angleichung von Wohlergehen berücksichtigt werden.

Schwere Behinderungen sieht Arneson als einen der wichtigsten Einwände gegen eine Gleichverteilung von Gütern (ebd.: 78). Menschen mit schweren Schädigungen müssten in bestimmten Bereichen wie z.B. für Hilfsmitteln mehr Ressourcen aufwenden als andere. Sie haben daher effektiv weniger Ressourcen, um eigene Lebenspläne zu erfüllen. Behinderungen seien in dieser Hinsicht analytisch äquivalent $\mathrm{zu}$,ungewollten teuren Vorlieben“ $\mathrm{zu}$ behandeln und in der Verteilung von Gütern zu berücksichtigen (ebd.: 78f., vgl. Anderson 1999a: 294). Soziale und biologische Faktoren würden in beiden Fällen die Ausbildung von Präferenzen im Leben beeinflussen (Arneson 1989: 79).

Richard Arneson hat seine Position später unter anderem in Reaktion auf Elizabeth Andersons Kritik abgeändert. In „Luck Egalitarianism and Prioritarianism“ meint er nun, dass innerhalb der Metrik des Wohlergehens ein „Verantwortung fördernder Prioritarismus“ die angemessenste Verteilungsregel sei (Arneson 2000: 340). Diesem Ansatz zufolge soll die Verbesserung des Wohlergehens der am schlechtesten Gestellten und derer, die für ihren Zustand nicht als verantwortlich gelten können Priorität über das der besser Gestellten haben (ebd.).

Ungleichheiten sind hier im prioritarischen Sinne einer Wohlergehensmetrik nur zu beanstanden, wenn sie für das Wohlergehen von Menschen schlecht sind (ebd.: 341, vgl. Parfit 1997: 214). Arneson entgeht damit unter anderem dem ,leveling down“-Einwand (bzw. „Angleichung nach unten“) - dem Problem, dass mehr Gleichheit auch durch eine Angleichung besser gestellter an schlechter gestellte Individuen erlangt werden kann (z.B. Parfit 1997: 211, u.a. auch von Joseph Raz und Larry Temkin formuliert). Gleichzeitig hat Gleichheit dadurch effektiv nur noch einen instrumentellen und nicht mehr intrinsischen Wert - sie ist wichtig in Hinblick auf wünschenswerte Konsequenzen, nicht aber in sich selbst (vgl. Parfit 1997: 206). 


\subsubsection{Kritik an Dworkin und Arneson}

Die Ressourcen- und Wohlergehens-Ansätze der Gerechtigkeit, wie sie hier exemplarisch anhand der Positionen von Ronald Dworkin und Richard Arneson dargestellt worden sind, stoßen im Hinblick auf das Phänomen Behinderung und vor allem auch die interaktionistischen Grundzüge eines Modells von Behinderung auf einige Probleme. Sowohl werden Aspekte der Problemkonstitution vernachlässigt, als auch bestimmte Lösungsansätze nicht berücksichtigt - insbesondere eine Intervention in Umfeld und Kontext von Menschen mit Schädigungen. Jerome Bickenbach kommentiert treffend:

\footnotetext{
„When justice theorists turn to disability, there is an immediate shift to impairments, understood as individual deficiencies. [...] An obsession with personal deficits and comparative well-being of persons with disability $[\ldots]$ certainly skews the discussion away from social and political organizations and their role in creating the disadvantages of disability, and the feasibility and social and economic advantages of doing so“ (Bickenbach 2009: 110f.).
}

Was Bickenbach generell formuliert, ist auch das Hauptproblem in Dworkins und Arnesons Ansätzen: die Reduktion von Behinderung auf die Dimension der Schädigungen. Dworkin und Arneson können aufgrund der Eigenschaften der jeweiligen Metriken diejenigen Aspekte, die mit dem interaktionistischen Charakter von Behinderung zusammenhängen, nicht berücksichtigen. In Dworkins Ansatz ist Behinderung ein Mangel an internen Ressourcen, der durch ein Mehr an sozialen Ressourcen entschädigt werden soll. Bei Arneson gefährden Behinderungen per se die Aussichten auf gleiches Wohlergehen, obwohl wie auch Dworkin anführt, dies höchstens ein statistischer Zusammenhang ist (Dworkin 1981b: 241). Dieser sollte jedoch wie bereits in Abschnitt 2.4 argumentiert nicht als konstitutiver Faktor des Phänomens Behinderung erachtet werden. Auch hier soll letztendlich ein Mehr an Ressourcen die Angleichung an die Lebensaussichten anderer Menschen, verstanden als Wohlergehen, ermöglichen.

Das führt zu den umfassenden Einwänden, die Elizabeth Anderson gegen die glücksegalitaristische Position erhoben hat. Angelika Krebs subsumiert die Einwände Andersons unter drei Aspekte: (i) Menschen, die an ihrer Situation selbst schuld sind, wird nicht geholfen, (ii) Menschen, die unverschuldet leiden, wird aus den falschen Gründen geholfen - weil sie relational schlechter dastehen als andere, und (iii) der Staat entmündigt und verletzt die Privatsphäre der Bürger, indem er versucht festzustellen, in welche Kategorie die jeweiligen Nachteile gehören - verschuldete oder unverschuldete (Anderson 1999a: 289, vgl. Krebs 2000: 21).

Hinsichtlich (i) ließe sich illustrativ das Beispiel zweier momentan prominenter Querschnittsgelähmter anführen: der in der Fernsehsendung „Wetten Dass...?“ verunglückte Samuel Koch und Philippe Pozzo di Borgo, auf dessen Autobiographie der Kinofilm „Ziemlich beste Freunde“ beruht. Beide sind aufgrund bewusst risikoreichen Verhaltens zu ihren Schädigungen gekommen: der eine aufgrund einer gefährlichen Wette, die einen Sprung über ein fahrendes Auto einschloss, der andere 
aufgrund eines schlecht vorbereiteten Gleitschirmflugs ${ }^{14}$. Sollten solche Menschen gerechtigkeitsbasierte Ansprüche auf Kompensation oder Unterstützung haben, also z.B. eine Kostenbeteiligung gesetzlicher Krankenkassen?

Dworkin und Arneson würden das vermutlich verneinen. Denn in beiden Fällen beruhen die Zustände auf freiwilligen Entscheidungen, für die die Individuen verantwortlich sind. In Dworkins Fall ist der unglückliche Ausgang des risikoreichen Verhaltens ein Fall von „,bad option luck“ (1981b: 293) bzw. kalkuliertem Pech und aus diesem Grund nicht qualifiziert für Kompensation. Für Arneson sind die Unfälle Resultat freiwilliger Entscheidungen und die Personen verantwortlich, weil sie das Risiko eines schlechten Ausgangs vernünftigerweise hätten voraussehen können (1989: 84, 88).

Die moralischen Intuitionen sind hier zumindest anders gelagert, und auch Menschenrechtsübereinkommen wie das ÜRMB spezifizieren ein Mindestmaß eines menschenwürdigen Lebens, unabhängig davon wie ein Mensch in eine missliche Lage geraten ist. Ladwig zieht aus der Auseinandersetzung mit den Glücksegalitaristen ebenfalls den Schluss, dass „Eigenverantwortung für folgenreiche Fehler nur auf einem Sockel zum Tragen kommen darf“ (2011a: 213), einem Sockel eines solchen menschenwürdigen Lebens. Die Frage der Verursachung von und Verantwortung für solche Benachteiligungen sollte dabei zunächst von der Frage nach der Zuständigkeit für die Behebung derselben getrennt werden (Wasserman 2001: 229).

Anderson kritisiert Arnesons und Dworkins Position u.a. als das „Problem der Vernachlässigung fahrlässiger Opfer“", die jedoch das liberale Gebot der Behandlung mit gleicher Achtung und Rücksicht, wie es explizit auch Dworkin vertritt, verletzen würde (Anderson 1999a: 295). Im Gegensatz zu anderen ,hardlinern' wie dem Gerechtigkeitstheoretiker Eric Rakowski, für den nun alles gesagt wäre, kämen Dworkin und Arneson immerhin zu dem Schluss, dass es unfair sein könne, solche Betroffenen komplett durch ein gesellschaftliches Sicherungsnetz fallen zu lassen - jedoch nur aus paternalistischen Gründen (ebd.: 300f.). Arneson z.B. meint, dass Menschen in ihrer Fähigkeit, vernünftig zwischen Optionen zu wählen, variieren können. In den genannten Fällen Fällen solle Kompensation für solch weniger vernünftige Menschen nicht in allen Fällen vorenthalten werden (Arneson 1989: 81, 86). Dworkin schränkt ein, dass es explizit aus ,paternalistischen Gründen“ sinnvoll sein kann, die Risiken, die Menschen eingehen dürfen, zu begrenzen (1981b: 295). Dieses „Problem des Paternalismus“ führe Anderson zufolge letztendlich zu einem respektlosen Umgang mit Bürgern, weil die Fälle, in denen ihnen trotz schlechter Entscheidungen geholfen wird, auf einem Urteil unzureichender Verantwortungsfähigkeit beruhen würden (Anderson 1999: 301).

Denjenigen, die nicht für eine bestehende Benachteiligung verantwortlich seien, werde hingegen aus Gründen geholfen, die ebenfalls gleiche Achtung und gleichen Respekt vermissen ließen (ii) -

14 Siehe z.B. das Interview mit Koch und Pozzo di Borgo im Spiegel vom 16.07.2012. 
aus einer Position der Unterlegenheit (ebd.: 302f.). Dies drücke sich, so Anderson, bei Dworkin etwa im moralisch fragwürdigen Neidprinzip aus, das eine Grundlage für die Zuteilung weiterer Güter sei - Neid auf eine bessere Position (ebd.: 307, vgl. Dworkin 1981b: 285). Bei Arneson findet sich dies in der Vermutung wieder, dass Menschen mit Schädigungen generell unter Wohlergehenseinschränkungen leiden (Anderson 1999: 304), bzw. unter selbigen leiden müssen, wenn sie einen Anspruch auf Kompensation geltend machen wollen.

Schließlich müsse der Staat für die Erörterung, ob einzelnen Menschen Kompensation zusteht oder nicht, moralisierende und übergriffige Urteile fällen (iii) (ebd.: 310f.). Im Fall von Arneson müsse der Staat z.B. beurteilen, ob Menschen für einzelne Präferenzen oder teure Vorlieben zur Verantwortung gezogen werden könnten oder nicht. Dieses Vorgehen würde Bürgern aber den schlechten Anreiz geben, Verantwortung abzustreiten und sich als hilflos darzustellen (ebd.: 311$)$.

Anderson ist sicherlich nicht in allen Kritikpunkten vollständig zuzustimmen. Es ist z.B. fraglich, ob das analytische Instrument der Neidfreiheit bei Dworkin tatsächlich schon Unterlegenheit impliziert, und Dworkin entgeht meiner Meinung nach auch recht deutlich Andersons Vorwurf, dass es sich bei seiner Position um eine ,starting gate theory“ handele (Anderson 1999: 308 und Fn. 64, anders aber explizit Dworkin 1981b: 309ff.). Auch Arneson wehrt sich in „Luck Egalitarianism and Prioritarianism “ gegen Teile von Andersons Kritik: Die Vorwürfe der Herablassung gegenüber Opfern bloßen Pechs sei unzutreffend und müßig - es sei einfach moralisch wertvoller Menschen zu helfen, die ceteris paribus für ihre benachteiligten Position nicht verantwortlich seien (Arneson 2000: 344f.). Die generelle Stoßrichtung der Kritik gegen die fragwürdigen Unterscheidungen zwischen verantworteten und nicht-verantworteten Entscheidungen der glücksegalitaristischen Position ist jedoch vor allem in Hinsicht auf das Thema Behinderung richtig.

Die verkürzte Problemkonstitution von Behinderung als Beeinträchtigung individueller Zustände schlägt sich auch in der gerechtigkeitstheoretischen Reaktion nieder. Sowohl in Dworkins als auch Arnesons Ansatz sollen Menschen mit Schädigungen durch ein Mehr an Ressourcen auf die Stufe von Ressourcengleichheit bzw. gleichen Wohlergehens angehoben werden - in Dworkins Ansatz explizit durch ein Mehr an ,whatever resources are privately owned by individuals“ (Dworkin 1981b: 283).

David Wasserman kritisiert, dass diese Ansätze die Umgebung als gegeben ansehen. Es sei zweifelhaft, dass sie Menschen mit Schädigungen darin unterstützen könnten, Teil sozialer und vor allem auch politischer Aktivitäten zu werden. Ressourcenbasierte Ansätze könnten letztendlich nur mit einem „,more of the same“, nämlich weiteren oder größeren Ressourcenbündeln antworten (Wasserman 2001: 237f.). Durch den Fokus auf Verteilungsanteile hätten die Ansätze Schwierigkeiten auch strukturelle und soziale Veränderungen zu begründen (Wasserman 1998: 166). Nicht alle Nachteile, selbst im Bereich unserer Zuständigkeit, sind jedoch als Ressourcendefizite zu verstehen (Ladwig 2011a: 196). Menschen mit Schädigungen werden auch durch Barrieren in der physischen Umgebung, Dis- 
kriminierung und soziales Stigma behindert, und verharren so potenziell in Zuständen sozialer Marginalisierung.

Die Konzeptionen blenden in diesem Sinne auch den in 2.1 und 2.7 angeführten Fakt der Kontinuität von Behinderung aus: beinahe jeder Mensch wird zu einem Zeitpunkt seines Lebens von Behinderung betroffen sein. Bickenbach kommentiert etwas polemisch, die Glücksegalitaristen suggerierten, dass es um „special needs for people who can’t make it in the real world“ ginge (Bickenbach 2009: 110). Das scheint aber auf der Annahme zu beruhen, dass es sowohl immer günstiger und effizienter, als auch politisch akzeptabler sei, mit Ressourcen auf individuelle Defizite zu reagieren, anstatt den Kontext zu verändern.

Der britische Philosoph Jonathan Wolff hingegen hat in Überlegungen, die auch konkrete policyMaßnahmen berücksichtigen, ausgeführt, dass es durchaus andere übergeordnete Strategien gibt, das Phänomen Behinderung politisch anzugehen: neben solchen des ,,personal enhancement“ wie bei Dworkin und Arneson, auch solche des ,, status enhancement “ - wie z.B. inklusivere Gesetze und eine Beseitigung von Barrieren in der öffentlichen Infrastruktur (Wolff 2009: 113). Letztere seien ersteren in vielen Fällen vorzuziehen, u.a. weil sie die Stigmatisierung einzelner Hilfeempfänger vermeiden können und oft auch Menschen ohne Schädigungen zugutekommen - z.B. weil sie das Risiko sozialer Marginalisierung auch für andere vulnerable Bevölkerungsgruppen verringern können und weil Rampen statt Treppen auch Personen mit Fahrrad oder Kinderwagen nützen (ebd.). Ressourcen- und Wohlergehensmetriken könnten jedoch keine guten Gründe anführen, warum solche Strategien im Zweifelsfall zu präferieren sind (ebd.: 115).

Zuletzt ist es oft nicht einleuchtend, an welchen Stellen Dworkin und Arneson die Grenze zwischen verantworteten und unverschuldeten Faktoren ziehen. Insbesondere sind Schädigungen weder äquivalent mit teuren Vorlieben, noch liegt der Unterschied zu ersteren immer darin, ob wir sie gewählt haben oder nicht. Ladwig argumentiert, dass bestimmte Dinge in den Bereich unserer Zuständigkeit fallen, auch wenn wir sie nicht gewählt haben - wie z.B. pflegebedürftige Eltern. Wir müssen sie dann dennoch verantworten, auch wenn wir uns nicht für sie entschieden haben (Ladwig 2011a: 194f.).

\subsection{Der Capability-Ansatz}

Die Entwicklung des Capability-Ansatzes kann, wie bereits erwähnt, als eine Reaktion auf Rawls $A$ Theory of Justice aufgefasst werden. Amartya Sen betont, dass den Capabilities als Gerechtigkeitsmetrik, wie auch als objektives Maß eines Niveaus an Wohlergehen gegenüber anderen Metriken eine breitere Informationsbasis zugrunde liegt, die eine größere Bandbreite unterschiedlicher Bedürfnisse berücksichtigen soll (1980: 217). 
Es sind ein paar kurze Anmerkungen zu den zentralen Begrifflichkeiten der Metrik nötig, weil hier die Möglichkeit inhaltlicher Missverständnisse besteht, als auch ein Ansatzpunkt berechtigter Kritik vorliegt. Während sich im Englischen , capability’ auch auf ein Alltagsverständnis stützt, weicht der Begriff doch im Gebrauch oft von diesem ab (Felder 2012: 105). Nicht erst im Deutschen erhält das Wort darüber hinaus eine Mehrdeutigkeit, etwa als ,Fähigkeit / Begabung / Potenzial / Vermögen’, die auch substanziell unterschiedliche Implikationen für die theoretische Auseinandersetzung etwa über die Charakterisierung und den Status des moralischen Personenbegriffs haben können (ob z.B. sehr kontrovers „Potenzialität“ als Grundlage eines solchen Status hinreichend sein kann, siehe z.B. Birnbacher 2006). Sen selbst hat später angemerkt, dass, obwohl er Capability letztendlich für geeignet und angemessen hält, es dennoch kein sehr ,attraktives“ Wort und potenziell missverständlich sei (1993: 30, 44 Fn. 36).

Ich werde Capability und auch functioning daher im Folgenden als termini technici behandeln und im englischen Original belassen. Das nicht zuletzt deswegen, weil die verschiedenen hier berücksichtigten Capability-Theoretiker den zwei Begriffen in ihren Konzeptionen mit unterschiedlichen Schwerpunkten abweichende Bedeutungen zuweisen. Während es Anderson und Nussbaum z.B. eher um Verwirklichungschancen hypothetischer Lebenssituationen geht, spielt bei Sen auch wesentlich die Fülle und Qualität tatsächlich zugänglicher Lebensentwürfe eine Rolle (Felder 2012: 97). Bei Sen korrespondieren Capabilities mit positiven Freiheiten, später deutlicher mit wertvollen Freiheiten die Freiheiten, die ein Mensch hat ein bestimmtes Leben zu führen (Sen 1993: 33ff.). Bei Nussbaum hingegen sind Capabilties überwiegend im Sinne hinreichender Schwellen aristotelisch interpretierter Bedingungen menschlichen Gedeihens ausgelegt worden, und bei Anderson schließlich als suffiziente Schwellen, um als demokratisch Gleicher gelten zu können.

Die Ansätze teilen aber auch eine Reihe grundlegender Annahmen. Die Capabilites sind im Gegensatz zu utilitaristischen und direkten Wohlergehens-Ansätzen in jedem Fall eine objektive Gerechtigkeitsmetrik. Das heißt, es geht um Verwirklichungschancen unabhängig von subjektiven Meinungen oder Vorlieben bestimmbarer functionings. Die functionings sind Zustände einer Person - ,things that he or she manages to do or be in leading a life“ (Sen 1993: 31). Alle Capability-Theoretiker betonen, dass Capabilities mit der Freiheit der Individuen verbunden sind, korrespondierende functionings umzusetzen oder nicht - sie seien in diesem Sinne nicht bevormundend oder repressiv paternalistisch (vgl. Ladwig 2011a: 199). Im Gegensatz zu anderen Metriken, auch Rawls Konzeption der Grundgüter, sei die Capability-Metrik besonders gut geeignet, Unterschiede zwischen Menschen und ihre Relevanz für gültige Ansprüche im Sinne der Verteilung von Gütern, Ämtern und Positionen zu berücksichtigen (z.B. Sen 1980: 215).

Sen macht Rawls den von anderen Capability-Theoretikern letztlich geteilten bekannten Vorwurf des Güterfetischismus: Nicht allein die Ausstattung mit Grundgütern kann über Benachteiligung 
Auskunft geben, stattdessen müssen Vorteile als Beziehungen zwischen Menschen und Gütern verstanden werden (Sen 1980: 216). Der Capabilities-Ansatz fragt danach, wozu Ressourcen oder Ressourcenbündel Menschen in bestimmten Kontexten tatsächlich befähigen. Elizabeth Anderson nennt diesen Zusammenhang das „Markenzeichen“ des Capability-Ansatzes - ein Kennzeichen, dessen es für die Berücksichtigung der Variation interner Voraussetzungen von Menschen bedarf:

\footnotetext{
„The hallmark of the capabilities approach is its sensitivity to variations in the abilities of individuals to convert resources into relevant functionings, which may be affected by internal variations, environmental features, and prevailing social norms“ (Anderson 2010: 87).
}

Dies ist der entscheidende Vorteil und das Alleinstellungsmerkmal der Capabilities-Metrik in Hinblick auf ein interaktionistisches Modell von Behinderung. Der Einwand der unterschiedlichen Fähigkeiten verschiedener Personen, aufgrund interner Merkmale Güter in wünschenswerte Zustände zu verwandeln, ist von Sen gegenüber Rawls am Beispiel Behinderung entwickelt worden (1980: 204). Die Umwandlung von Gütern in Capabilities variiert substanziell zwischen Personen: Der Status als Gleicher des einen Menschen mit bestimmten Ressourcen unter bestimmten Voraussetzungen ist nicht unbedingt der eines anderen (ebd.: 219). Das Differenzprinzip, in dem auf der Grundlage solcher Unterschiede keine Umverteilung gerechtfertigt ist, berücksichtigt u.a. dieses „,conversion handicap “, wie es Sen später genannt hat (z.B. 2004: 3), nicht ausreichend.

\subsubsection{Sen}

In seinem klassischen Text Equality of What (1980) argumentiert Amartya Sen unter anderem anhand des Beispiels Behinderung, dass die Grundgütermetrik von Rawls zu inflexibel sei. Sie könne nicht berücksichtigen, dass die Fähigkeit, zugeteilte Ressourcen in gewünschte Zustände umzusetzen, zwischen Personen signifikant variiert (ebd.: 203). Um die Capabilities von Menschen zu messen, müssen Sen zufolge neben Faktoren wie Einkommen und Ressourcen auch die persönlichen Charakteristiken der Individuen berücksichtigt werden und dazu zählen unter anderem Schädigungen, die zu Behinderungen führen können (2004: 3). Sen hat später expliziter hinzugefügt, dass Variationen in dieser Fähigkeit der „Konversion“ von Ressourcen in gewünschte Zustände letztendlich allgegenwärtig seien und zu unterschiedlichem Ausmaß zwischen allen Personen aufträten (2010: 248).

Sen ist der Auffassung, dass aus Gründen der Gerechtigkeit sichergestellt werden sollte, dass Menschen unter der Berücksichtigung ihrer Differenzen gleiche „sets“ von Capabilities aufweisen - wenngleich der Ansatz nicht auf diese egalitäre Interpretation beschränkt sei (1993: 50). Beispiele von functionings, deren Verwirklichungsbedingungen aus Gründen der Gerechtigkeit hergestellt werden sollen, umfassen für Sen grundlegende Faktoren wie das Verfügen über adäquate Nahrungsmittel, Unterkunft und Bewegungsfreiheit sowie komplexere functionings wie Selbstachtung, Partizipation 
in einer Gemeinschaft und die bei Adam Smith entliehene Möglichkeit, in der Öffentlichkeit ohne Scham auftreten zu können (1980: 218, 1993: 36f.). Das schließt für Sen ebenso wie Smith ein, dass Zustände wie Armut immer auch relational und komparativ bestimmt werden müssen, weil sich mit einer wachsenden Wirtschaftleistung die Bedingungen für solch ein schamfreies Auftreten verändern können. Die Capability-Sets einzelner Menschen werden bei Sen daher im Gegensatz zu Nussbaum und Andersons Auffassung relativ bestimmt.

Anders als Nussbaum und auch Anderson hat Sen allerdings keine konkrete Liste von Capabilities erstellt. Solch eine Konkretisierung solle nicht wie bei Nussbaum als Teil der begründenden Theorie selbst konzipiert werden, sondern als Teil öffentlicher Diskurse (Sen 2010: 284f.). Nussbaums Liste hingegen beruhe auf der aristotelischen Annahme einer einzelnen Liste und auf einer Konzeption menschlicher Natur, die potenziell „tremendously overspecified“ sei (Sen 1993: 47). Der Capabilities-Ansatz könne auf unterschiedlichen Wegen spezifiziert werden. Sen will damit Raum lassen für die demokratische Legitimierung durch eine begründete Zustimmung. Die Anwendung des Ansatzes soll in Abhängigkeit vom jeweiligen kulturellen Kontext geschehen (Sen 1980: 219). Das bewegt seinen Ansatz aber potenziell in Richtung eines (Werte-)Relativismus - es wird nicht klar, zu welchem Ausmaß von grundlegenden menschlichen Capabilties abgewichen werden kann.

In Abgrenzung zu Ressourcenmetriken und auch Rawls meint Sen ähnlich wie Arneson, dass Ressourcen und Einkommen nicht Zwecke, sondern Mittel von Verteilungsgerechtigkeit seien. Die Verwendung einer Ressourcen- oder Grundgütermetrik führe zu einem „Fetischismus“ bzw. einem „fetishist handicap “ hinsichtlich der nicht in sich wertvollen Güter - so Sen mit marxistischem Einschlag (1980: 216, 218). Die Capabilities-Metrik sei Rawls Grundgütermetrik darin überlegen, die Besserstellung von Personen mit sehr verschiedenen individuellen Merkmalen zu beurteilen - nicht aber unbedingt in allen anderen Belangen (Sen 2010: 242).

Gegenüber Wohlergehensmetriken hat Sen ebenso eine ganze Reihe von Bedenken - ich möchte hier nur auf den noch nicht angesprochenen und für die Capabilties-Metrik oft charakteristischen Einwand der ,, adaptive preferences “ bzw. dem ,taking pleasures from small mercies“ eingehen (Sen 2004: 6, vgl. z.B. Nussbaum 2007: 73). Dieser Einwand kann eigentlich von allen Vertretern objektiver Metriken gegenüber subjektiven Metriken erhoben werden: Er greift das Phänomen auf, dass manche Präferenzen unter ungerechten Bedingungen formuliert werden bzw., dass benachteiligte Menschen ihre Erwartungen oft schlechten Umständen anpassen (ein potenzielles Problem für die Behandlung des Phänomens Behinderung, ich komme im nächsten Abschnitt darauf zurück). Nussbaum nennt das Beispiel indischer Frauen in ländlichen Gebieten, die aufgrund geringer Erwartungen und fehlenden Wissens und Bildung ihre Gesundheitszustände durchschnittlich positiver einschätzten als die Männer am selben Ort. Die Einschätzungen passten sich nach Bildungsprogrammen auf ein niedrigeres aber realitätsnäheres Niveau an (Nussbaum 1992: 230f.). Gerechtigkeitstheoretische 
Ansätze sollten daher zumindest nicht ausschließlich auf subjektiven Bewertungen beruhen, weil die Beurteilenden sich in ihren Präferenzen und Interessen in diesem Sinne irren können. Und Gerechtigkeit sollte sich unter anderem aus diesem Grund gegen Unterdrückung wenden, auch wenn diese keine akuten Einschränkungen des Wohlergehens zur Folge hat (Sen 1980: 212, anders z.B. Arneson 2000: 341).

Behinderung, bzw. im Original der „cripple“ (1980: 203), ist für Sen ein Beispiel einer „case implication critique“ - die Auswirkungen eines moralischen Prinzips anhand eines besonders deutlichen Beispiels darzustellen und dann in Hinsicht auf unsere moralischen Intuitionen zu überprüfen (ebd.: 197). Das Beispiel Behinderung führt ihn dazu, eine utilitaristische Metrik und zu geringerem Ausmaß auch Rawls Grundgüter als Grundlage von Verteilungsgerechtigkeit abzulehnen. Das Differenzprinzip würde zwar z.B. das Problem teurer Vorlieben lösen, es würde aber einem Menschen mit Schädigung keine besondere Behandlung zusprechen - und das sei zu streng (ebd.: 214f.).

Wie bereits zitiert, will Rawls Behinderung als einen für Fragen der Gerechtigkeit weniger signifikanten Aspekt ausklammern, um eine klarere und weniger überladene Antwort zu ermöglichen - bzw. aus Gründen „methodologischer Einfachheit“ wie Nussbaum formuliert (2006: 107). Menschen seien aber tatsächlich sehr unterschiedlich, so Sen, und ihre Interessen variierten deutlich. Der Ausschluss von solch signifikanten Unterschieden führe Rawls zu einer ,partially blind morality“ (Sen 1980: 216).

Menschen mit Schädigung litten tendenziell an jeweils einem ,earning “ und ,,conversion handicap “, wie es Sen formuliert hat (2004: 3). Unter ersterem versteht Sen die auch empirisch fundierbaren Tatsachen, dass es Menschen mit Schädigungen auf der einen Seite meist schwerer fällt, Arbeit zu finden und diese auf der anderen Seite auch über ihre volle Lebensspanne auszuüben. Als ,,conversion handicap “ bezeichnet Sen indessen das Phänomen, dass Menschen mit Schädigungen meist schlechter Ressourcen in tatsächliche wünschenswerte Funktionen umwandeln können - z.B. Geld in Vorstellungen des guten Lebens (ebd.: 2004: 3, vgl. Sen 1980: 219). Das earning handicap wird also durch das conversion handicap noch verstärkt (Sen 2004: 7). Das grundlegende Problem der Grundgüter sei ebenso wie bei den Ressourcen einer Ressourcenmetrik, dass sie unabhängig von den Eigenheiten der Individuen bestimmt werden und deswegen im Gegensatz zur Capabilities-Metrik den Personen extern seien (ebd.: 6).

Im Gegensatz zu nur negativen etwa libertären Rechten der nicht-Intervention und Vergleichen von Grundgüterausstattungen sei die Capabilities-Metrik zusätzlich grundlegend mit der Vorstellung positiver Freiheit verbunden (Sen 1993: 30, ausführlicher Sen 1992: 56ff.). Sen meint, dass Freiheit von intrinsischer Wichtigkeit für Wohlergehen sei, weil etwa freies Handeln und die Möglichkeit, zwischen einer Reihe von Lebensplänen wählen zu können, selbst förderlich sei (ebd.: 39). Das unterscheide den Capability-Ansatz etwa auch von dem, was Cohen „midfare“ genannt hat (ebd.: 43). 


\section{Kritik}

Der gegenüber Dworkin und Arneson erhobene Vorwurf der Reduktion auf die Dimension der Schädigung trifft letztendlich auch auf Sen zu. Wenn bei Sen die Rede von Behinderung ist, dann geht es ähnlich wie bei Arneson und Dworkin stets um individuelle Zustände. Sen berücksichtigt nicht den konstitutiven Aspekt des Umfelds für das Entstehen und Bestehen von Behinderungen und spricht daher auch nicht von Lösungsansätzen innerhalb desselben. In „,Inequality Reexamined“ grenzt Sen Behinderung explizit als Differenzen in persönlichen Charakteristiken von solchen der natürlichen und sozialen Umwelt ab: „But in addition to these differences in natural and social environments and external characteristics, we also differ in our personal characteristics (e.g. age, sex, physical and mental abilities) [...] A disabled person cannot function in the way an able-bodied person can“" (ebd.). Es ist zwar zutreffend, dass Unterschiede in physischen und geistig-psychischen Fähigkeiten persönliche Charakteristiken sind, der Umstand der Beeinträchtigung der , behinderten Person' ist aber bei Sen überwiegend diesen Variationen geschuldet. Behinderung wird damit im Sinne der in Abschnitt 2.4 vorgenommenen Differenzierung wesentlich auf die Dimension der Schädigung reduziert. Es geht bei Sen im Fall von Behinderung zwar um die Relation zwischen Gut und Individuum, nicht aber um den konstitutiven Zusammenhang zwischen Individuum und Kontext. Behinderung bleibt somit auch bei Sen wesentlich eine individuelle, persönlichen Zuständen geschuldete Eigenschaft.

Auch Nussbaum kritisiert, dass Sen Behinderung als eine ,natural asymmetry“ behandelt. Die Einschränkung der Fähigkeit zu Mobilität eines Paraplegikers hat neben seiner Schädigung eben auch damit zu tun, was für eine Infrastruktur zur Verfügung steht und ob auch Ansprüche auf die Modifizierung des Umfelds bestehen (Nussbaum 2007: 165f.). Nussbaum ist der Überzeugung, dass die Kritik radikaler sein müsste: Sen scheint zu meinen, dass wenn das ,, conversion handicap “ in der Verteilung von Reichtum und Einkommen ausgeglichen wird, den Ansprüchen von Menschen mit Schädigungen Genüge getan ist (ebd.).

Sen (wie allerdings auch Nussbaum, 1992: 234) hat ursprünglich vorgeschlagen, Rawls Ansatz durch die Capabilities-Metrik zu ergänzen: Rawls solle die Capabilities statt der Grundgüter zur Grundlage für Verteilungsgerechtigkeit machen. Nussbaum zumindest hat ihre Meinung später geändert: Die Capabilities-Metrik müsse als irreduzibel plural aufgefasst werden, sie sei nicht im Sinne einzelner quantitativer Standards kommensurabel (Nussbaum 2007: 166). Dieses Problem bleibt für Sen in Hinsicht auf seine komparative Konzeptualisierung gleicher Capabilities bestehen: Ob nun als Teil des Differenzprinzips oder einer anders gearteten Angleichung verschiedener Capability-Sets es ist unklar, wie die relativen sozialen Positionen angesichts pluraler heterogener Kriterien bestimmt werden sollen (so z.B. auch Pogge 2010: 51f.). 
Die angesprochene Betonung positiver Freiheiten führt Sen zum Teil zu eigenartigen Formulierungen, die verschiedentlich als Indiz aufgefasst werden, dass Sen den Freiheitsbegriff überstrapaziert. So meint Sen etwa, die Freiheit von Hunger oder Malaria sei nicht nur rhetorisch zu verstehen, sondern Maßnahmen zur Verhinderung solcher Übel seien tatsächlich als Zunahme an Freiheit aufzufassen (Sen 1993: 44). Wie verschiedentlich eingewendet wurde, hat Freiheit aber mit Optionen zu tun, etwas zu tun oder sein lassen zu können - wenn Malaria hingegen ausgerottet ist, dann gibt es auch keine Möglichkeit mehr sich mit der Krankheit anzustecken (z.B. Cohen 1993: 22ff., Ladwig 2011a: 200). David Wasserman kommentiert, dass Freiheit bei Sen Gefahr laufe, selbst zu einem ,fetischisierten Gut' zu werden. Sen erwecke zum Teil den Eindruck, dass generell ein Leben je besser wäre, desto mehr Freiheit es aufweise (Wasserman 2001: 234).

Ein mögliches Problem für das Thema Behinderung ist in diesem Zusammenhang, dass viele Formen von Schädigungen Einschränkungen von Freiheiten bedeuten - ein Schrumpfen des Horizonts an Möglichkeiten. Das wäre im Zweifelsfall auch für die Beurteilung der Leben von Menschen mit Schädigungen folgenreich: Es würde etwa in Schrammes Beispiel des zeugungsunfähigen Mannes implizieren, dass dieser ein schlechteres Leben führt - selbst wenn er gar keinen Kinderwunsch hat. Solch eine Begrenzung von Freiheitsgraden muss aber noch nicht unbedingt eine Einschränkung der Lebensqualität zur Folge haben, wenn dennoch ausreichend wertvolle Optionen bestehen (Schramme 2003a: 188, Wasserman 2001: 198).

Damit hängt ein weiteres potenzielles Problem zusammen, das in Hinsicht auf das Phänomen der „, adaptive preferences “ entstehen kann. Unter dem Schlagwort ,, disability paradox “ wird manchmal das Phänomen angesprochen, dass Menschen mit Schädigungen selbst bei objektiv relativ schlechten Lebensumständen meist eine relativ hohe subjektive Zufriedenheit angeben (z.B. Bickenbach 2012a: 117, Felder 2012: 94). Es kann dafür verschiedene Gründe geben - bei einer schweren durch einen Unfall erworbenen Schädigung zum Beispiel die Erleichterung, überlebt zu haben. In diesem Sinn könnte die Anpassung an objektive schlechte Umstände ein Grund für die subjektive Beurteilung sein. Es ist aber potenziell stigmatisierend, Menschen mit Schädigungen generell zu unterstellen, dass sie sich in ihrer Zufriedenheit täuschen (Wasserman 2001: 230, 238). Eine Capabilities-basierte Antwort könnte sein, dass man zumindest dann nicht mehr von ,,adaptive preferences “ ausgehen sollte, wenn eine Stufe der Suffizienz einzelner als objektiv wertvoll erachteter Capabilities erreicht ist.

Dadurch, dass Sen keine konkreten Capabilities spezifiziert, ist sein Ansatz aber letzten Endes kaum operationalisierbar. Auch Nussbaum meint, dass Sen zu „unbestimmt“ sei. Die Gewährleistung effektiver Freiheiten müsse mit Verteilungsfragen verknüpft werden (Nussbaum in „Women and Human Development “ 2000: 13, nach Felder 2012: 101). Angesichts konfligierender Ansprüche auf knappe Ressourcen braucht es eine Priorisierung von entscheidenden und moralisch erheblichen Ansprüchen (vgl. Arneson 1989: 91, Ladwig 2011a: 200, vgl. Scanlon 1975: 660f.). Ohne solche Un- 
terscheidungen wird nicht klar, für die Behebung welcher Nachteile die Gesellschaft oder das Individuum zuständig ist (Ladwig 2011a: 201). Es ist z.B. unklar, wie Sen mit unterschiedlichen Begabungen umgehen würde (Bickenbach 2009: 117): Sollen alle ,natürlichen' Ungleichheiten kompensiert werden? Es bleibt zudem bei Sen offen, welche konkreten Maßnahmen gegen verschiedene Formen des Unrechts und der Benachteiligung ergriffen werden sollten (so z.B. auch Eurich 2011: 90).

\subsubsection{Nussbaum}

Die amerikanische Philosophin Martha Nussbaum hat den Capabilities-Ansatz zum Teil komplementär mit Amartya Sen entwickelt. Hinsichtlich der Verteilungsregel bleibt Nussbaum letzten Endes agnostisch. Auf der einen Seite bestimmt sie im Sinne einer Suffizienzregel eine Schwelle von Capabilities, die durch einen gerechten Staat mindestens hergestellt werden müsse. Auf der anderen Seite ist sie zum einen wie Rawls der Überzeugung, dass manche Rechte (gefasst als Capabilities), wie etwa Redefreiheit und Religionsfreiheit, und auch einige functionings, wie ihrer Auffassung nach menschliche Würde und Selbstachtung gleichverteilt werden sollten (Nussbaum 1992: 221, 291ff.; Nussbaum 2007: 172, 179). Zum anderen schränkt Nussbaum explizit ein, dass ihre Interpretation des Capabilities-Ansatz keine vollständige Theorie sozialer Gerechtigkeit sei, weil sie z.B. nichts über Ungleichheiten jenseits der Capabilites-Schwellen aussage (Nussbaum 2007: 75).

Nussbaums Strategie der Begründung und der Bestimmung des Inhalts der Capabilities-Liste hat sich im Lauf der Zeit geändert: Zuerst sollte sie noch stärker auf einer Genealogie menschlicher Tugenden und anthropologischen Annahmen eines aristotelischen Essentialismus beruhen (Nussbaum 1992, 1993), später dann zunehmend auf einem Begriff menschlicher Würde und den sie garantierenden Bedingungen (Nussbaum 2007 und vor allem 2008). Der Stellenwert aristotelischer Annahmen hat dabei kontinuierlich abgenommen (so auch Nussbaum selbst 2008: 361). Insbesondere hat sich Nussbaums Ansatz zunehmend von einem substanziell anthropologischen zu einem Ansatz entwickelt, der zunehmend die „Sprache der Menschenrechte“ aufnimmt (Graumann 2011: 156, ähnlich auch Jörke 2005: 97, Ladwig 2009: 263, Strassenberger 2009: 136).

In der im Rahmen des Themas zentralen Monographie, Frontiers of Justice (2007), in der Nussbaum auf das Thema Behinderung als eines von drei ,Grenzgebieten' der Gerechtigkeit eingeht, beruhen die „Schwellen“, die den Anwendungsbereich der Capabilities abstecken, auf der so genannten Speziesnorm (ebd.: 182). Sie ist eine Interpretation der aristotelischen Vorstellungen menschlichen Gedeihens. Das menschliche Gedeihen soll zwar nicht mehr wie bei Aristoteles in einem einzelnen selbstgenügsamen und vollkommenen ergon bestehen. Aristoteles zufolge ist die spezifische Bedeutung des höchsten Gutes Glück (eudaimonia) für den Menschen ein vernunftgeleitetes tätiges Leben, in Abgrenzung zum mit den Pflanzen gemeinsamen Wachsen und sich-ernähren und dem mit den 
Tieren gemeinen Leben der Wahrnehmung (Aristoteles NE 1097 b 21ff., Gigon 2004: 115ff.). Bei Nussbaum hingegen soll durch die Sicherstellung einer Schwelle an Capabilities ein Raum für eine Vielzahl von Vorstellungen menschlichen Gedeihens eröffnet werden (Nussbaum 2007: 182).

Um zu ihrer konkreten Liste von Capabilities zu gelangen, die für ein gutes menschliches Leben nötig seien, unterscheidet Nussbaum ursprünglich zwei solche Schwellen. Eine erste Schwelle bestimmt eine Grenze zu Zuständen, die aufgrund signifikanter Einschränkungen in Capabilities „subhuman“ seien (2007: 278): Nussbaum zählt dazu Menschen im Wachkoma und Kinder mit Anenzephalie (1992: 221; 2007: 181). Solche Individuen würden, so Nussbaum, in wohl kaum einer Gesellschaft als menschlich bezeichnet werden und sie sind daher auch keine Gerechtigkeitssubjekte (1992: 219, 1993: 264). Nussbaum fragt im Anschluss, welche Capabilities ein menschliches Leben von solch ,verarmten` Zuständen unterscheiden würden. Die zweite, höher angesiedelte Schwelle von Capabilties soll ein gutes menschliches Leben ermöglichen. Diese zweite Schwelle soll für die meisten Capabilities durch eine gerechte Politik sichergestellt werden.

Nussbaum hat die Capabilities-Liste zunächst in Anlehnung an die von Aristoteles benannten Sphären menschlicher Erfahrung und die ihm zufolge jeweils korrespondierenden Tugenden konzipiert (Aristoteles: NE 1115a 4 ff., Gigon 2004: 163, vgl. Nussbaum 1993: 246). Ihre eigene Liste ist Nussbaum zufolge „evaluativ“ bzw. „ethisch evaluativ“ (Nussbaum 1992: 208, 2007: 181), es soll sich um Capabilities handeln, die normativ grundlegend sind (ebd.). Sie soll prinzipiell änderbar und unabgeschlossen sein - Nussbaum hat wie erwähnt über die Zeit Änderungen an ihr vorgenommen (und etwa Verweise auf Rechte eingeführt) (vgl. 1992: 222 und 2007: 76ff.). Ähnlich wie Sen ist Nussbaum der Meinung, dass die Liste in verschiedenen Nationen oder Kulturkreisen unterschiedliche Spezifizierungen zulässt - jedoch innerhalb der Grenzen der konkretisierten Liste. Die in der Liste aufgeführten Capabilities könnten daher, so hofft Nussbaum, Teil eines Rawls'schen overlapping consensus sein (2006: 6, 182).

Im Gegensatz zu Rawls ,thin theory of the good“" verteidigt Nussbaum eine ,thick, vague theory of the good“ - diese sei nicht prozedural, sondern „ergebnisorientiert““(1992: 214, 2007: 81). Konkret gibt Nussbaum an, für die Erstellung der Liste von Capabilities auf menschliche Erzählungen verschiedener Epochen und Gesellschaften zurückzugreifen, etwa auf antike Mythen und auch Romane (Nussbaum 1992: 215, vgl. auch Jörke 2005: 93). Den konkreten Versuch einer solchen intern oder „empirisch“ (Nussbaum 1992: 207f.) rekonstruierenden Perspektive ist Nussbaum aber bisher schuldig geblieben, wie Straßenberger anmerkt (2009: 132).

Behinderung sei ein drängendes Problem moderner Gerechtigkeitstheorien - das Ziel müsse die Inklusion von Menschen mit Schädigungen als gleichberechtigte Bürger sein (Nussbaum 2007: 20). Wie in der Kritik an Rawls bereits angeführt, entwickelt Nussbaum ihre Position hinsichtlich der Inklusion von Menschen mit Schädigung wesentlich aus einer Kritik an Rawls und auch ande- 
ren vertragstheoretischen Positionen. Die Gründe für die Nicht-Inklusion seien in der Struktur der Vertragstheorien zu finden, vor allem in den bereits erwähnten Umständen der Gerechtigkeit und der kurzschließenden Anwendung der vereinbarten Prinzipien nur auf die idealisierten Kooperationsteilnehmer (vgl. Krebs 2000: 12).

\section{Kritik}

Viele Aspekte von Nussbaums Konzeption des Capability-Ansatzes sind im Hinblick auf das hier skizzierte interaktionistische Modell und die Natur des Phänomens Behinderung vorteilhaft: Der konstitutive Aspekt des physischen und sozialen Kontexts wird betont, die Allgegenwärtigkeit von Schädigungen und deren kontinuierlicher Übergang zu ,normalen Zuständen“ werden hervorgehoben. So schreibt Nussbaum etwa: „A good analysis requires recognizing the many varieties of impairment, need, and dependency that "normal" human beings experience, and thus the real continuity between "normal" lives and those of people with lifelong mental disabilities" (2007: 92).

Im Zusammenhang mit der Frage danach, wie produktiv Menschen mit Schädigungen sein könnten und welchen Beitrag sie zum Gemeinwesen im Sinne reziproker Beziehungen leisten, betont Nussbaum, dass dies grundlegend von der Art und dem Ausmaß von Fördermaßnahmen abhänge. Dies gelte z.B. für Menschen mit so genannten Lernbehinderungen und, wie Nussbaum anführt, auch für Menschen mit Trisomie 21 - von denen lange angenommen wurde, dass sie wenig EntwicklungsPotenzial hätten oder denen einfach unterstellt wurde, dass sie dumm seien (ebd.: 190).

Schließlich entwirft Nussbaum, wie bereits erwähnt, eine konkrete Liste von Capabilities, die als Grundlage der Regulierung konkurrierender Verteilungsansprüche dienen kann. Die Gerechtigkeitssubjekte sollen entlang dieser Verwirklichungschancen zunächst bis zu einer Stufe der Suffizienz befähigt werden, was (genau wie bei Anderson im folgenden Abschnitt) im Gegensatz zu Sens Konzeption keine relationalen Urteile über bessere oder schlechtere ,Capability-sets ' involviert. Nussbaum entgeht damit dem Problem der Kommensurabilität solch heterogener Kriterien und muss zu keinem Urteil über den relativen Wert eines bestimmten Bündels von Capabilities gelangen.

Gleichzeitig ist Nussbaums aristotelisch geprägte Interpretation der Capabilities für die Berücksichtigung von Menschen mit Schädigungen in mehreren Hinsichten problematisch. Insbesondere sind die formulierten Capabilities zu anspruchsvoll-spezifisch, sie beruhen vor allem im Kontext Behinderung auf zweifelhaften normativen Grundlagen und sie schließen aufgrund der Bestimmung eines Minimums an Capabilities für die Berücksichtigung als Gerechtigkeitssubjekt entlang der ,Speziesnorm` einige Individuen mit schweren Schädigungen nicht nur als Rechtssubjekte, sondern gleich als Gattungsmitglied aus.

Sowohl die Schwelle zu einem ,menschlichen Leben“als auch die zu einem, guten menschlichen Leben` ist in manchen Fällen schwerer Schädigungen kaum sinnvoll zu erreichen (vgl. Kuhlmann 
2005: 44ff.). Die Schwelle zu einem menschlichen Leben sollte anfangs durch die ,architektonischen Capabilities“ „practical reason“ und ,affiliation“ (Nussbaum 1992: 222), später durch eine „hinreichend bedeutsame Gruppe“ von Capabilities (Nussbaum 2007: 181) und schließlich durch das Vorhandensein mindestens einer , basic capability “ sichergestellt werden (Nussbaum 2008: 363) - in jedem Fall aber soll diese für Kinder mit Anenzephalie und Menschen im Wachkoma unerreichbar sein (ebd.). Bestimmte moralpragmatische Grenzen, wie etwa der Ganzhirntod, sind vermutlich für den Status als Rechtssubjekt letztendlich sinnvoll (vgl. Ladwig 2011a: 112) - der Ansatzpunkt derselben ist jedoch zumindest nicht unumstritten ${ }^{15}$ und es bleibt die Frage, ob diese Individuen gleich aus der Menschengemeinschaft ausgeschlossen werden sollten (so aber Nussbaum 1992: 192, 2007: 278) ${ }^{16}$.

Auch bezüglich der Schwelle zu einem , guten menschlichen Leben` lässt sich zumindest fragen, ob solche Capabilities, wie uneingeschränkt alle Sinne gebrauchen zu können, in Sorge um und in Beziehung zu Tieren und Pflanzen leben zu können und nicht frühzeitig sterben zu müssen (ebd.: 76ff.), tatsächlich in jedem Fall die Unmöglichkeit eines guten Lebens präjudizieren. Andreas Kuhlmann kommentiert, dies sei „offensichtlich unangemessen und öffnet der Abwertung konkreter Lebensstile Tür und Tor“ (2005: 46).

Schließlich ist äußerst fragwürdig, warum ein gattungsgebundenes Kriterium wie die Speziesnorm normativ grundlegend bedeutsam sein soll. Bernd Ladwig etwa wendet ein: „Das bloße Faktum, ein menschliches Genom zu besitzen, ist moralisch ohne Belang. Es wäre merkwürdig, ein Lebewesen von anderer Art, das erleben, streben, überlegen, handeln und moralische Rücksicht nehmen könnte wie wir, allein deshalb schlechter zu behandeln, weil es das ,falsche“ Erbgut hätte“ (2011b: 6f.). Nussbaums Ansatz wird durch die normative Grundlage der Speziesnorm und die zwei aus ihr hervorgehenden gehaltvollen Schwellen eines menschlichen und guten menschlichen Lebens effektiv wieder exkludierend gegenüber bestimmten Fällen von Schädigungen (vgl. Graumann 2011: 156).

\subsubsection{Anderson}

Elizabeth Anderson verbindet in ihrer Skizze eines gerechtigkeitstheoretischen Ansatzes die Capability-Metrik mit einer Suffizienzregel der Verteilung. Ihre Konzeption ist für das Thema Behinderung insofern von Belang, als dass sie eine normativ sparsamere Metrik als Nussbaum verwendet und durch die Sicherstellung suffizienter Schwellen politisch relevanter Capabilites einen Status als glei-

15 Siehe z.B. Andreas Zieger (2007): Wie viel Gehirn braucht ein Mensch? Anmerkungen zum Anencephalie-Problem aus beziehungsmedizinischer Sicht, in: Behindertenpädagogik, Band 46, Nr. 1, S. 52-66.

16 Schramme bezieht sich in anderem Zusammenhang auf Avishai Margalits Beschreibung von Demütigung als genau diesen Prozess des Ausschlusses von Menschen aus der Menschengemeinschaft und erinnert daran, dass dies unter anderem während des Holocausts massenhaft geschah (Schramme 2006: 234f.) - nicht zuletzt gegenüber geschätzt 300.000 ermordeten Menschen mit Schädigungen (Quelle: Pressemitteilung des Behindertenbeauftragten, zugänglich unter: http://www.behindertenbeauftragter.de/SharedDocs/Pressemitteilungen/DE/2012/ PM01_Gedenkveransaltung_cm.html, zuletzt eingesehen: 19.02.2013). 
cher demokratischer Bürger gewährleisten soll - gegen Zustände der sozialen Marginalisierung und Unterdrückung (Anderson 1999a: 312). Im Gegensatz zu Nussbaums Speziesnorm und auch Rawls Konzeption der moralischen Person impliziert Andersons Ansatz vor allem keinen Ausschluss aus der Gruppe der Gerechtigkeitssubjekte, wenn eine bestimmte substanzielle Schwelle von Eigenschaften nicht erreicht wird. Darüber hinaus stellt sie die Sensibilität des Capability-Ansatzes sowohl für Variationen individueller Fähigkeiten als auch solche des sozialen und physischen Kontextes heraus, und betont, dass die gerechtigkeitstheoretische Reaktion auf Beeinträchtigungen in diesen Dimensionen der Art der Benachteiligung angemessen sein sollte.

Andersons Variante des Capability-Ansatzes ist außerdem interessant, weil er eine Art Hybrid darstellt zwischen einer Verteilungstheorie der Gerechtigkeit und einer Theorie gerechter sozialer Beziehungen (Pogge 2010: 47, Wasserman 2001: 243). In letzterer Hinsicht hat Andersons Ansatz Gemeinsamkeiten mit Positionen einiger feministischer Kritikerinnen purer Verteilungsansätze der Gerechtigkeit - konkreter mit Konzeptionen etwa von Iris Young und Nancy Fraser, auf die sie sich auch bezieht. Die Ansätze teilen unter anderem eine Sensibilität dafür, dass von Ungerechtigkeiten oft diejenigen betroffen sind, die aufgrund bestimmter Kriterien als nicht gleichwertig erachtet werden, und dass die politische Philosophie sich das Wissen sozialer Bewegungen über reale vielfältige Formen der Ungerechtigkeit und Unterdrückung zunutze machen sollte (Horn / Scarano 2002: 348). Ihr Ansatz sei offen dafür, Ansprüche auf Anerkennung mit in den Ansatz zu integrieren, so Anderson (1999a: 314). Gleichzeitig vermeidet Anderson einige der problematischeren Implikationen vor allem von Youngs Ansatz, weil sie darauf beharrt, dass Adressaten der Gerechtigkeit nicht soziale Gruppen, sondern Individuen sind, und eine gerechte Verteilung bestimmter Güter für den Status als gleicher Bürger zumindest als Mittel essentiell ist (ebd.).

Um es mit dem Titel von Andersons Artikel zu formulieren: , What is the point of equality? “, bzw. warum nun eigentlich Gleichheit? Anderson zufolge soll zwischen Bürgern „demokratische Gleichheit“" hergestellt werden. Bei Rawls ist demokratische Gleichheit diejenige, die durch das Differenzprinzip hergestellt wird (Rawls 2003: 43 und ausführlicher Rawls 1971: 75ff.). Für Anderson bedeutet democratic equality hingegen, dass die Forderung nach gleicher Achtung und Rücksicht und gleichem Status als demokratischer Bürger durch einen effektiven Zugang zu den sozialen und materiellen Bedingungen für die Freiheit der Bürger hergestellt werden soll (Anderson 1999a: 288). Dafür sei nur ein relativ eng begrenzter Rahmen kollektiv bereitgestellter Güter nötig.

Anderson skizziert ebenso wie Nussbaum eine Liste von Capabilities. Die Komponenten der Liste sollen aber im Gegensatz zu Nussbaum nicht von einer umfassenden aristotelischen Vorstellung menschlichen Gedeihens angeleitet sein. Stattdessen sollen solche Capabilities grundlegend sein, die auf der einen Seite Individuen dazu befähigen, unterdrückende oder dominierende Beziehungen zu vermeiden, und auf der anderen Seite den Status als gleicher Bürger in einem demokratischen Staat 
ermöglichen (ebd.: 316). Anderson zählt dazu drei Dimensionen individueller Verwirklichungschancen: solche, die das Individuum als Mensch betreffen, als Teilnehmer in einem System kooperativer Produktion und als Bürger in einem demokratischen Staat (ebd.: 317f.). Zum ersten Aspekt zählen konkret etwa ein effektiver Zugang zu den Mitteln biologischer Subsistenz - wie z.B. Nahrungsmittel, Bekleidung, Unterkunft und medizinische Versorgung - und zu grundlegenden Bedingungen menschlicher Handlungsfähigkeit - wie etwa das Wissen um Umstände und Optionen, die Fähigkeit über Mittel und Zwecke zu reflektieren, die psychologischen Bedingungen für Autonomie, sowie Freizügigkeit und Gedankenfreiheit. Zum zweiten Aspekt der Teilnahme an einem System kooperativer Produktion rechnet Anderson u.a. einen Zugang zu Bildung, freie Berufswahl, einen effektiven Zugang zu Produktionsmitteln (wenn auch nicht unbedingt anteiliges Eigentum an denselben!), ein Recht für die eigene Arbeit faire Entlohnung zu erhalten und Anerkennung der eigenen produktiven Beiträge (ebd.: 318). Zu den Capabilities hinsichtlich des Bürgerstatus in einem demokratischen Staat gehören schließlich z.B. die Rechte politischer Teilhabe und die sozialen Bedingungen dafür, durch andere als Gleicher anerkannt zu werden. Wie bei Sen und Nussbaum sollen jeweils Capabilities, also die Chancen auf die Verwirklichung der functionings, nicht die functionings selbst sichergestellt werden. Diese Capabilities schulden sich sowohl die Bürger untereinander als auch der Staat den Bürgern.

Nun könnte man meinen, dass diese Capabilities, wenn sie weiter ausbuchstabiert werden, doch auch nicht sehr viel weniger anspruchsvoll als etwa die von Nussbaum seien (so z.B. Kuhlmann 2005: 46). Erstens beziehen sich Andersons Capabilites aber eher auf grundlegendere ,Kapazitäten“, die den Weg der Umsetzung offener lassen - also z.B. Zugang zu Bedingungen menschlicher Handlungsfähigkeit anstatt einer konkreten Capability, alle Sinne zu nutzen (siehe Nussbaum 2007: 76). Auch Schramme gelangt zu bestimmten objektiv und nicht-relational bestimmbaren „,basalen Fähigkeiten“ - wie etwa einem Vermögen zur Kommunikation, ohne deswegen spezifisch hören können zu müssen, und einem Vermögen zur Mobilität, ohne deshalb funktionell und strukturell uneingeschränkte Beine haben zu müssen (Schramme 2003a: 189, 2006: 223ff.). Gerade grundlegende Gerechtigkeitsansprüche seien nicht komparativer Natur, weil sie uns aufgrund personaler Eigenschaften und nicht in Abhängigkeit von Eigenschaften anderer zustünden (ebd.: 226f.). Diese Grundbedürfnisse müssten erfüllt werden, um ein „,nicht misslingendes Leben zu ermöglichen“(ebd.).

Zweitens und grundlegender präjudiziert Andersons Ansatz im Gegensatz zu Nussbaum aber auch nicht, dass man jenseits einer bestimmten Schwelle von Capabilities nicht mehr als voll menschlich gelten könne. Das Ziel ist nicht, ein „gutes Leben“ im Sinne einer umfassenden Vorstellung des Guten zu ermöglichen, sondern die Bedingungen für einen Status als gleicher Bürger - eine „,unktionell demokratische Orientierung“, wie Wasserman formuliert (2001: 244). Das ist wie erwähnt insbesondere 
im Hinblick auf eine Konzeption politischer Gerechtigkeit relevant, weil eine so geartete Befähigung die Teilnahme an politischen Verteilungskämpfen ermöglicht (Schramme 2006: 222, s.o) ${ }^{17}$.

Es wird auch kein anspruchsvoller moralischer Personenstatus vorausgesetzt, um überhaupt als Subjekt demokratischer Gerechtigkeit gelten zu können. Anderson gesteht zwar zu, dass es in den schwersten Fällen vielleicht zusätzlicher Prinzipien bedarf - nämlich v.a. in solchen, in denen es keinerlei Potenzial gibt, in gleichberechtigten Beziehungen zu anderen zu stehen (1999a: 331). Zunächst besteht aber die Präsumption, dass jeder Mensch unter Berücksichtigung der individuellen Fähigkeiten im jeweiligen Kontext bis zu einer suffizienten Schwelle befähigt werden sollte.

Im Hinblick auf die in 2.7 skizzierten Grundzüge eines interaktionistischen Modells von Behinderung lässt sich außerdem wie erwähnt betonen, dass Anderson besonders die Zusammenhänge zwischen individuellen Zuständen und den Bedingungen des sozialen und physischen Kontexts der Capability-Metrik hervorstellt:

„The fundamental difference between capability theorists and resource theorists lies $[\ldots]$ in the degree to which their principles of justice are sensitive to internal individual differences, and environmental features and social norms that interact with these differences" (ebd., Hervorhebung V.D.).

Der Unterschied zu Ressourcenmetriken bestehe hingegen nicht darin, was verteilt werden könne - dies sind auch im Capabilities-Ansatz nicht die Fähigkeiten oder Tätigkeiten selbst, sondern die Ressourcen, die Menschen zu Tätigkeiten und Lebensweisen befähigen sollen. Auch hinsichtlich der Zwecke der Verteilung bestehe kein grundlegender Dissens gegenüber anderen objektiven Metriken: Es gehe letztendlich um die Befriedigung grundlegender objektiver menschlicher Bedürfnisse.

\section{Fazit}

Der vorliegenden Arbeit lag die Frage zugrunde, welche gerechtigkeitstheoretische Metrik und Verteilungsregel auf der Basis eines angemessenen wissenschaftlichen Verständnisses von Behinderung geeignet ist, die Rechte und Pflichten von Menschen mit funktionellen und strukturellen Beeinträchtigungen zu inkludieren. Im ersten Teil der Arbeit habe ich für ein wissenschaftliches Modell von Behinderung mit interaktionistischen Grundzügen argumentiert. Behinderung ist darin konzipiert als ein relationales Phänomen, das in der Wechselwirkung zwischen (a) sich auf ontologisch objektive Zustände funktioneller und struktureller Einschränkungen beziehende ,Schädigungen', und (b) physischen und sozialen Bedingungen des Kontexts, ent- und besteht (2.).

17 Als konkretes Policy-Beispiel könnte man die Ausdehnung des Wahlrechts anführen, um die willkürliche Ungleichbehandlung auf Grundlage auch finanziell bedingter Betreuungsstufen abzuschaffen - siehe z.B. Leander Palleit (2011): Gleiches Wahlrecht für alle? Menschen mit Behinderungen und das Wahlrecht in Deutschland, zugänglich unter: www.institut-fuer-menschenrechte.de/uploads/tx_commerce/ policy_paper_18_gleiches_wahlrecht_fuer_alle.pdf, zuletzt eingesehen: 19.02.2013. 
In empirischer Hinsicht wurde herausgestellt, dass Behinderung eine weit verbreitete Dimension potenzieller sozialer Benachteiligung und Marginalisierung ist, deren Ausmaß entscheidend mit Variationen in den Bedingungen sozialstaatlicher Institutionen und ökonomischer Zustände korreliert. Die Inzidenz von Behinderung wird aufgrund global alternder Bevölkerungen und der nicht zuletzt damit verbundenen Zunahme chronischer Erkrankungen weltweit weiterhin stark zunehmen (2.1).

Es wurde differenziert, dass Behinderungen auf einer Vielzahl verschiedener Formen von Schädigungen beruhen können und bemängelt, dass große Teile der gerechtigkeitstheoretischen Literatur die Relevanz einiger dieser grundlegenden Unterschiede nicht berücksichtigen. Insbesondere beruht die wissenschaftliche Behandlung oft auf der Gleichsetzung von Behinderung mit schweren körperlichen und weniger häufig auch schweren geistig-psychischen Schädigungen (2.2).

Eine angemessene theoretische Auseinandersetzung, so das Argument, müsse aufgrund der dargestellten Komplexität des Phänomens auf einem wissenschaftlichen Modell beruhen und nicht auf einer zwangsläufig einseitigen Definition. Ein solches Modell kann die vielschichtigen Zusammenhänge des Komplexes Behinderung auf wesentliche Modellkomponenten vereinfacht sinnhaft darstellen und zugleich die Konsistenz operationaler Definitionen verschiedener Politikfelder untereinander gewährleisten (2.3).

Alle in der wissenschaftlichen Literatur ernsthaft diskutierten Modelle beziehen sich dabei mindestens auf die Unterscheidung der Komponente ,Schädigung' (Impairment) vom übergeordneten Konzept der Behinderung. Schädigungen werden dabei fast universell als zeitlich relativ stabile individuelle Zustände funktioneller und struktureller Beeinträchtigungen gefasst. Viele Ansätze bringen solche Beeinträchtigungen darüber hinaus mit der Abweichung von typischen menschlichen Eigenschaften in einem Gesundheitskontext in Verbindung (2.4).

Anhand einer in der Literatur oft vorgenommenen idealtypischen Gegenüberstellung zwischen einem so genannten ,medizinischen' und ,sozialen Modell' von Behinderung wurde dafür plädiert, Behinderung weder auf die Komponente der Schädigung zu reduzieren, noch die Relevanz dieser individuellen Zustände für die Einschränkung relevanter Handlungsmöglichkeiten zu bestreiten. Unter dem medizinischen Modell wird meist die monokausale und zwingende Folgerung, von Schädigungen als inhärente Übel auf Behinderungen zu schließen, verstanden. Extremere Varianten des sozialen Modells stellen hingegen zwischen Behinderung und der Komponente Schädigung gar keine kausale Verbindung her - Einschränkungen seien stattdessen wesentlich einem beeinträchtigenden sozialen Umfeld geschuldet (2.5).

Vor allem Anhänger des sozialen Modells charakterisieren Behinderung und zum Teil auch Schädigungen als soziale Konstruktionen. Demgegenüber wurde hier argumentiert, dass Schädigungen entlang epistemisch objektiver Kriterien identifiziert werden können und in der Regel auf ontologisch 
objektive Zustände verweisen. Die Zuschreibung von Schädigungen und Behinderungen beruht zwar auf umkämpften, nicht aber willkürlichen sozialen Normen (2.6).

Als Ergebnis der Auseinandersetzung mit dem Phänomen und Begriff Behinderung wurde die Angemessenheit eines Modells von Behinderung mit interaktionistischen Grundzügen konstatiert. Dieses Grundverständnis, das in einem zunehmenden Teil der Literatur vertreten wird und auch als Grundlage der Internationalen Klassifikation der Funktionsfähigkeit, Behinderung und Gesundheit der WHO sowie des Übereinkommens über die Rechte von Menschen mit Behinderungen der UN dient, betont die konstitutive Relevanz sowohl von Schädigungen als auch von behindernden und befähigenden Kontexten für das Be- und Entstehen von Behinderung (2.7).

Der zweite Teil der Arbeit hat sich mit der Darstellung und Kritik verschiedener gerechtigkeitstheoretischer Positionen und der jeweiligen Behandlung des Phänomens Behinderung sowie den Implikationen für die theoretische Inklusion von Menschen mit Schädigungen auf der Basis eines interaktionistischen Grundverständnisses beschäftigt. Die Bedeutung von Gerechtigkeit wurde für die Zwecke der Arbeit auf die Dimension politischer Gerechtigkeit eingegrenzt und mit John Rawls als Angelegenheit in erster Linie sozialer Institutionen aufgefasst. Darüber hinaus folgt die Auseinandersetzung dem vornehmlich zeitgenössischen Verständnis, die Frage nach politischer Gerechtigkeit weitgehend als eine der gerechten Verteilung gesellschaftlicher Güter und Lasten zu formulieren (3.).

Die Diskussion nimmt ihren Ausgang bei Rawls, an dessen Konzeption sich die Kontroverse um die gerechtigkeitstheoretische Inklusion von Menschen mit Schädigungen wesentlich entzündet hat (3.1). Es wird argumentiert, dass der Ansatz der Gerechtigkeit als Fairness mit einer Grundgütermetrik und einer komplexen Kombination aus einer prioritären und Suffizienzregel der Verteilung Menschen mit Schädigungen aus mindestens drei Gründen nicht theoretisch inkludieren kann (3.2).

Erstens beruht Rawls Konzeption auf einem engen vertragstheoretischen Verständnis von Reziprozität und zu geringerem Ausmaß auch auf der Erwartung gegenseitiger Vorteile der Kooperation. Zumindest Menschen mit schweren körperlichen und geistig-psychischen Schädigungen werden diese Voraussetzungen aber in den meisten Fällen nicht erfüllen können (3.2.1).

Zweitens setzt die Berücksichtigung als Subjekt von Gerechtigkeit als Fairness den mit anspruchsvollen Bedingungen verknüpften Status einer moralischen Person voraus. Vor allem in Hinsicht auf Menschen mit geistig-psychischen Schädigungen ist aber fraglich, ob diese die von Rawls anvisierten ,zwei moralischen Vermögen', die freie und gleiche Bürger aufweisen sollen, erlangen können. Rawls Position lässt sich mindestens in diesem Punkt im Hinblick auf das rechtfertigende Konzept des Überlegungsgleichgewichts auch immanent kritisieren (3.2.2).

Drittens schließlich benötigt das von Rawls ersonnene Differenzprinzip trotz eigentlich pluraler Grundgüter einen einzelnen quantitativen Index relativer sozialer Positionen, um die jeweils am wenigsten Begünstigten in einer Gesellschaft identifizieren und durch Umverteilung besserstellen zu 
können. Der von Rawls gewählte Index des Einkommens und Vermögens kann aber spezielle Bedürfnisse vieler Menschen mit Schädigungen - wie etwa besonders kostspielige Medizin oder Hilfsmittel - nicht berücksichtigen und belässt solche Menschen daher effektiv mit sehr viel weniger Ressourcen für die Umsetzung von Lebenszielen (3.2.3).

Im Anschluss an Amartya Sens Kritik an Rawls in seinem Artikel „Equality of What? “ und die dadurch mit angestoßene gleichnamige gerechtigkeitstheoretische Debatte haben unter anderem die so genannten ,Glücksegalitaristen' versucht, diesen Makel bei Rawls aufzugreifen. Die hier eigenständig berücksichtigten Positionen von Ronald Dworkin und Richard Arneson können aus Sicht des Themas Behinderung als eine Antwort auf die konstatierte fehlende Sensibilität von Rawls Position gegenüber den besonderen Ansprüchen von Menschen mit Schädigungen verstanden werden.

Die glücksegalitaristische Position verbindet die Ansicht, dass Gerechtigkeit wesentlich in der Angleichung von Lebensaussichten besteht, mit der Vorstellung, dass nur unverdiente Vor- und Nachteile kompensiert werden sollten. Während Nachteile, die aus unverschuldeten Umständen und bloBem Zufall (,, brute luck“) erwachsen, kompensiert werden sollten, gilt das für verschuldete Entscheidungen und kalkulierte Risiken (,option luck“), für die Menschen jeweils die Verantwortung tragen sollen, nicht (3.3).

Ronald Dworkin vertritt eine Ressourcenmetrik verbunden mit einer - im Sinne der in dieser Arbeit bei Anderson entliehenen Nomenklatur - egalitären Verteilungsregel: Ressourcen sollen so verteilt werden, dass verschiedene Menschen effektiv gleiche Ressourcen für die Umsetzung von Lebensplänen haben (3.3.1). Richard Arneson hingegen favorisiert eine objektive Variante einer Wohlergehensmetrik, die er zuerst mit einer egalitären und später mit einer prioritären Verteilungsregel verbunden hat (3.3.2).

Dworkin und Arneson können im Gegensatz zu Rawls beide berücksichtigen, dass eine Behandlung von Menschen mit Schädigungen aufgrund dieser individuellen Zustände eine Ungleichverteilung von Gütern erfordern kann, um Ressourcengleichheit bzw. gleiche Chancen auf Wohlergehen herzustellen. Im Hinblick auf das zugrunde gelegte interaktionistische Modell von Behinderung stoßen beide Ansätze aber auf das Problem, dass sie Behinderung auf die Dimension der Schädigung reduzieren. Menschen mit Schädigungen sollen Kompensation erhalten, weil sie in ihren ,internen Ressourcen' eingeschränkt sind, bzw. weil sie aufgrund ihrer Schädigung geringere Aussichten auf gleiches Wohlergehen haben. Zugleich können Dworkin und Arneson aufgrund dieser einseitigen Problemkonstitution auch nur einseitige Lösungen anbieten: ein Mehr an individuellen Gütern. Gerechtigkeitsbasierte Interventionen im physischen und sozialen Kontext, wie etwa einen Barriereabbau in der öffentlichen Infrastruktur, können diese Positionen jedoch nicht berücksichtigen.

Die glücksegalitaristische Position ist darüber hinaus aus weiteren Gründen für die gerechtigkeitstheoretische Inklusion von Menschen mit Schädigungen problematisch, wie anhand Elizabeth 
Andersons umfangreicher Kritik in ihrem Artikel „, What is the point of Equality? “ ausgeführt wurde: (i) Menschen, die für ihre Schädigung verantwortlich gemacht werden können, haben keine gerechtigkeitsbasierten Ansprüche; (ii) Menschen, die ihre Schädigungen unverschuldet ,erworben' haben, wird aus den falschen Gründen geholfen - weil sie relational ,ärmer dran' sind als andere; und schließlich (iii) durch das Unterfangen, zwischen unverschuldeten und verschuldeten Nachteilen aufgrund von Schädigungen zu unterscheiden, würde ein dieser Gerechtigkeitskonzeption folgender Staat die Privatsphäre der Bürger verletzen und sie letztendlich entmündigen (3.3.3).

Die Entwicklung der Capabilities-Metrik kann im Sinne des Anstoßes in Sens „Equality of What? “ ebenso wie der Glücksegalitarismus als eine Reaktion auf die fehlende Berücksichtigung der normativen Relevanz der Variation individueller Zustände in Rawls gerechtigkeitstheoretischer Konzeption verstanden werden. Die Capabilities-Metrik soll Verwirklichungschancen für die Erlangung bestimmter objektiv wünschbarer grundlegender functionings herstellen - moralisch erheblicher Tätigkeiten und Lebenssituationen. Elizabeth Anderson hebt hervor, dass es das Kennzeichen des Capability-Ansatzes sei, danach zu fragen, wozu ein Bündel zugeteilter Güter Menschen mit bestimmten internen Voraussetzungen vor dem Hintergrund eines partikularen Kontextes sozialer Faktoren und Umweltfaktoren effektiv befähigt (3.4).

Sen zufolge leiden Menschen mit Schädigungen aufgrund ihrer Schädigung an einem conversion und earning handicap: Sie können schlechter als andere Menschen Ressourcen in wünschenswerte functionings umwandeln und es würde ihnen schwerer fallen, Arbeit zu finden und diese über eine volle Lebensspanne auszuüben. Sen verbindet die Capabilities-Metrik mit einer egalitären Verteilungsregel: Es sollen effektiv gleiche Sets von Capabilities für verschiedene Menschen sichergestellt werden.

Wenn auch zu einem geringeren Maß, so hat Sens Ansatz dennoch ähnlich wie Dworkins und Arnesons Position das Problem, Behinderung weitgehend auf den individuellen Zustand der Schädigung zu reduzieren. Wenn conversion und earning handicap durch Umverteilung ausgeglichen werden, dann haben Menschen mit Schädigungen in Sens Ansatz vermutlich keine weiteren gerechtigkeitsbasierten Ansprüche (3.4.1).

Martha Nussbaum verfolgt im Gegensatz zu Sen eine substanziell aristotelische Interpretation des Capability-Ansatzes. Gerechtigkeit bestehe in der Sicherstellung einer Schwelle konkret aufgelisteter Capabilities, die Nussbaum zuletzt in Hinsicht auf die so genannte Speziesnorm spezifiziert hat. Diese soll ein gutes menschliches Leben im Sinne bestimmter gattungseigener Bedingungen menschlichen Gedeihens ermöglichen. Bezüglich der Verteilungsregel bleibt Nussbaum letzten Endes agnostisch - jenseits der suffizienten Schwelle von Capabilities lässt sie die Frage nach einer gerechten Verteilung offen. Nussbaums Ansatz schließt im Gegensatz zu Sen ausdrücklich auch Interventionen im physischen und sozialen Umfeld als gerechtigkeitsbasierte Ansprüche ein. 
Nussbaum ist für ihre Konzeption jedoch auch vielfach kritisiert worden - besonders vor dem Hintergrund des Themas Behinderung sind die substanziellen und anspruchsvollen aristotelisch inspirierten Capabilities problematisch, weil viele der Verwirklichungschancen von Menschen mit einer Vielzahl von Schädigungen in keiner bedeutsamen Weise erreicht werden können. Insbesondere ist es heikel, nicht nur ähnlich Rawls den Status als Gerechtigkeitssubjekt, sondern gleich den Status als Gattungsmitglied vom Erreichen einer minimalen Schwelle dieser umfassenden Capabilities abhängig machen zu wollen - die darüber hinaus auf überaus zweifelhaften normativen Grundlagen beruhen (3.4.2).

Elizabeth Anderson spezifiziert ähnlich Nussbaum eine konkrete Liste von Capabilities, die jedoch normativ sparsamer ist. Sie soll nur die Sicherstellung der Bedingungen umfassen, die für einen Status als demokratisch gleicher Bürger nötig sind. Anderson fordert jenseits einer Gleichverteilung allgemeiner Rechte explizit nur eine Stufe der Suffizienz der so spezifizierten Capabilities. Die Verteilung von Gütern sei für die Sicherstellung demokratischer Gleichheit letztendlich nur instrumentell - das normative Prinzip, das der Suffizienzregel zugrunde liegt, ist nicht-relational bestimmt. Andersons Ansatz kann ähnlich der Konzeption von Nussbaum eine gerechtigkeitsbasierte Intervention in allen Komponenten eines Behinderungsmodells mit interaktionistischen Grundzügen stützen und schließt eine leichte Präsumption zugunsten der Intervention in Kontexten ein, weil diese im Zweifelsfall nicht nur Menschen mit Schädigungen zugutekommen (3.4.3).

Auch Anderson ist für ihren Ansatz selbstverständlich kritisiert worden. Die spezifizierten Capabilities seien immer noch zu anspruchsvoll, um Menschen mit schweren Schädigungen berücksichtigen zu können (Bickenbach 2009, Kuhlmann 2005, Wasserman 2001). Es sei fraglich, ob suffiziente Schwellen demokratischer Gleichheit überhaupt diskret bestimmt werden könnten, oder ob solche Festlegungen nicht notwendig komparative und relationale Urteile involvierten (Bickenbach 2009, Wasserman 2001, vgl. Sen 1993). Schließlich würde Anderson ähnlich anderen Capability-Theoretikern keine Obergrenze für Investitionen in die Befähigung von Individuen setzen können, die verantwortungslos handeln oder die eine Stufe der Suffizienz grundlegender Eigenschaften nicht absehbar erreichen können (Arneson 2000, Pogge 2010).

Auf einen Teil der Einwände kann geantwortet werden und hat Anderson auch reagiert (siehe 1999b). So kann das Problem der oberen Grenze der Unterstützung etwa insofern Capability-immanent gelöst werden, als dass es das Verbleiben ausreichender Mittel für die Befriedigung konkurrierender Ansprüche zur Bedingung für weitere Leistungen macht. Andersons Liste ist auch tatsächlich immer noch recht anspruchsvoll - der qualitative Unterscheid zu Nussbaum besteht aber darin, dass ein Nichterreichen irgendeiner Schwelle keine Implikationen für den Status als Mitglied in der Gemeinschaft von Gerechtigkeitssubjekten hat. Ein moderater Perfektionismus im Sinne einer , funktionellen demokratischen Orientierung' (s.o.) kann zumindest in liberalen Demokratien auch legitim 
sein. Er könnte mit Rawls als Teil eines „classical republicanism“ verstanden werden (Rawls 2003: 144), der es für die Sicherstellung politischer und auch nichtpolitischer Freiheiten als erforderlich erachtet, dass Bürger aktiv partizipieren. Diese Perspektive wäre damit immer noch deutlich abzugrenzen von einer umfassenderen aristotelischen oder auch platonischen Vorstellung des Menschen als zoon politikon (bzw. in der Nikomachischen Ethik als wesenhaft ,auf die staatliche Gemeinschaft ausgelegt", Aristoteles NE 1169 b 16, Gigon 2004: 323), die das Politische als einen essentiellen Wesenszug oder Zweck des Menschen erkennen will.

Andere Herausforderungen, wie etwa eine einleuchtende korrespondierende Verteilung auch gesellschaftlicher Lasten, scheinen größere Hürden darzustellen. Auch die Frage nach der nicht-relationalen Bestimmbarkeit absoluter Schwellen wird höchst kontrovers diskutiert ${ }^{18}$. Nicht zuletzt bezieht sich auch Anderson auf die Fähigkeit, in der Öffentlichkeit ohne Scham auftreten zu können - was, wie Sen mit Adam Smith betont, intim mit relationalen Urteilen verbunden ist. Eine zufriedenstellende Klärung dieser Fragen würde den Rahmen der Arbeit aber sprengen. Es ist insofern sinnvoll, sich wie Nussbaum auf eine agnostische Position zurückzuziehen - auch wenn ein solcher „Schrumpfegalitarismus“ eigene Probleme mit sich bringt (siehe dazu Krebs 2000: 20, 29). David Wasserman konstatiert:

„Even if Anderson underestimates the extent to which democratic equality requires harsh trade-offs [...] she has made great strides in elevating people with impairments from caricatures and cameo appearances to equal partnership in a challenging intellectual and moral inquiry" (Wasserman 2001:245).

Was die Fragestellung der gerechtigkeitstheoretischen Inklusion von Menschen mit Schädigungen auf der Basis eines Modells von Behinderung mit interaktionistischen Grundzügen angeht, ist Andersons Konzeption jedoch aus den genannten Gründen momentan zumindest die attraktivste Ausgangsbasis.

18 Zur Übersicht: die Beiträge in Krebs (2000), Bejahung einer solchen Möglichkeit neben Anderson z.B. Krebs selbst 2000, Parfit 1997, Schramme 2006 - Verneinung z.B. Ladwig 2004, 2011a; Pogge 2010, Tugendhat 1993, Wasser$\operatorname{man} 2001$. 


\section{Literaturverzeichnis}

Altman, Barbara (2001): Disability Definitions, Models, Classification Schemes, and Applications, in: Gary Albrecht / Katherine Seelman / Michael Bury (Hrsg.): Handbook of Disability Studies. Thousand Oaks: Sage, S. 97-123.

Anderson, Elizabeth (1999a): What is the Point of Equality? in: Ethics, Band 109, Nr. 2, S. 287-337. Anderson, Elizabeth (1999b): Reply to Arneson, Christiano, Sobel - Symposium 'What is the Point of Equality', zugänglich unter: http://www.brown.edu/Departments/Philosophy/ bears/9912ande.html, zuletzt eingesehen: 18.07.2012.

Anderson, Elizabeth (2010): Justifying Capabilities, in: Harry Brighouse / Ingrid Robeyns (Hrsg.): Measuring Justice - Primary Goods and Capabilities. Cambridge: Cambridge University Press, S. 81-101.

Arneson, Richard (1989): Equality and equal Opportunity for Welfare, in: Philosophical Studies, Band 56, Nr. 1, S. 77-93.

Arneson, Richard (2000) - Luck Egalitarianism and Prioritarianism, in: Ethics, Band 110, Nr. 2, S. 339-349.

Becker, Lawrence (2005): Reciprocity, Justice, and Disability, in: Ethics, Band 116, Nr. 1, S. 9-39.

Bickenbach, Jerome (2009): Disability, Non-Talent and Distributive Justice, in: Kristjana Kristiansen / Simo Vehmas / Tom Shakespeare (Hrsg.): Arguing about Disability - Philosophical Perspectives. Oxon: Routledge, S. 105-124.

Bickenbach, Jerome (2012a): Ethics, Law, and Policy - Disability Key Issues and Future Directions Series. Thousand Oaks: Sage.

Bickenbach, Jerome (2012b): The International Classification of Functioning, Disability and Health and its Relationship to Disability Studies, in: Nick Watson / Alan Roulstone / Carol Tomas (Hrsg.): Handbook of Disability Studies. Oxon: Routledge, S. 51-67.

Birnbacher, Dieter (2006): Der künstliche Mensch - ein Angriff auf die menschliche Würde? in: Ders.: Bioethik zwischen Natur und Interesse. Frankfurt a.M.: Suhrkamp, S. 77-101.

Birnbacher, Dieter (2009): Was kann die Anthropologie zur Politik in ihrer theoretischen Fundierung beitragen? in: Dirk Jörke / Bernd Ladwig (Hrsg.): Politische Anthropologie - Chancen und Grenzen anthropologischer Begründungsmuster. Baden-Baden: Nomos, S. 179-195.

BMAS (2011): Zahlen und Fakten zur Lebenssituation von Menschen mit Schädigungen, zugänglich unter: http://www.bmas.de/shareddocs/downloads/de/zahlen-und-fakten-lebenssituation-menschen-mit-behinderungen.pdf, zuletzt eingesehen: 12.09.2012. 
Brighouse, Harry / Robeyns, Ingrid (2010) (Hrsg.): Measuring Justice - Primary Goods and Capabilities. Cambridge: Cambridge University Press.

Buchanan, Allen / Brock, Dan / Daniels, Norman / Wikler, Daniel (2000): From Chance to Choice Genetics and Justice. Cambridge: Cambridge University Press.

Cloerkes, Günther (2007): Soziologie der Behinderten - Eine Einführung, 3. Auflage. Heidelberg: Universitätsverlag Winter.

Cohen, Gerald (1989): On the Currency of Egalitarian Justice, in: Ethics, Band 99, Nr. 4, S. 906-944.

Cohen, Gerald (1993): Equality of What? On Welfare, Goods, and Capabilities, in: Martha Nussbaum / Amartya Sen (Hrsg.): The Quality of Life - A Study prepared for the World Institute for Development Economics Research (WIDER) of the United Nations University. Oxford: Oxford University Press, S. 9-30.

DIMDI (2005): Internationale Klassifikation der Funktionsfähigkeit, Behinderung und Gesundheit. Herausgegeben vom Deutschen Institut für Medizinische Dokumentation und Information - WHO-Kooperationszentrum für das System Internationaler Klassifikationen, zugänglich unter: http://www.dimdi.de/dynamic/de/klassi/ downloadcenter/icf/endfassung, zuletzt eingesehen: 12.09.2012.

Dworkin, Ronald (1981a): What is Equality? Part 1: Equality of Welfare, in: Philosophy and Public Affairs, Band 10, Nr. 3, S. 185-246.

Dworkin, Ronald (1981b): What is Equality? Part 2: Equality of Resources, in: Philosophy and Public Affairs, Band 10, Nr. 4, S. 283-345.

EU (2004): Gemeinsamer Bericht der Kommission und des Rates über die soziale Eingliederung, zugänglich unter: http://ec.europa.eu/employment_social/soc-prot/ soc-incl/joint_rep_de.htm, zuletzt eingesehen: 12.09.2012.

Eurich, Johannes (2011): Gerechtigkeit für Menschen mit Schädigung - Ethische Reflexionen und sozialpolitische Perspektiven. Frankfurt a.M.: Campus.

Felder, Franziska (2012): Inklusion und Gerechtigkeit - Das Recht behinderter Menschen auf Teilhabe. Frankfurt a.M.: Campus.

Felkendorff, Kai (2003): Ausweitung der Behinderungszone - Neuere Behinderungsbegriffe und ihre Folgen, in: Günther Cloerkes (Hrsg.): Wie man behindert wird - Texte zur Konstruktion einer sozialen Rolle und zur Lebenssituation betroffener Menschen. Heidelberg: Universitätsverlag Winter, S. 53-81.

Gigon, Olof (Hrsg.) (2004): Aristoteles - Die Nikomachische Ethik, 6. Auflage. München: Deutscher Taschenbuch Verlag. 
Graumann, Sigrid (2010): Assistierte Freiheit - Autonomie und Gerechtigkeit für behinderte Menschen, in: Elisabeth List / Harald Stelzer (Hrsg.): Grenzen der Autonomie.

Weilerswist: Velbrück Wissenschaft, S. 215-235.

Graumann, Sigrid (2011): Assistierte Freiheit - Von einer Behindertenpolitik der Wohltätigkeit zu einer Politik der Menschenrechte. Frankfurt a.M.: Campus.

Hollenweger, Judith (2003): Behindert, arm und ausgeschlossen - Bilder und Denkfiguren im internationalen Diskurs zur Lage behinderter Menschen, in: Günther Cloerkes (Hrsg.): Wie man behindert wird - Texte zur Konstruktion einer sozialen Rolle und zur Lebenssituation betroffener Menschen. Heidelberg: Universitätsverlag Winter,

S. 141-164.

Horn, Christoph / Scarano, Nico (Hrsg.) (2002): Philosophie der Gerechtigkeit - Texte von der Antike bis zur Gegenwart. Frankfurt a.M.: Suhrkamp.

Hull, Richard (1998): Defining Disability - A Philosophical Approach, in: Res Publica, Band 4, Nr. 2, S. 199-210.

Jörke, Dirk (2005): Politische Anthropologie - Eine Einführung. Wiesbaden: VS Verlag für Sozialwissenschaften.

Kastl, Jörg (2010): Einführung in die Soziologie der Behinderung. Wiesbaden: VS Verlag.

Krebs, Angelika (2000): Einleitung - Die neue Egalitarismuskritik, in: Dies. (Hrsg.):

Gleichheit oder Gerechtigkeit. Frankfurt a.M.: Suhrkamp.

Kuhlmann, Andreas (2003): Schmerz als Grenze der Kultur, in: Ders. (2010): An den

Grenzen unserer Lebensform - Texte zur Bioethik und Anthropologie. Frankfurt a.M.:

Campus, S. 173-181.

Kuhlmann, Andreas (2005): Behinderung und die Anerkennung von Differenz, in: Ders.: (2010): An den Grenzen unserer Lebensform - Texte zur Bioethik und Anthropologie. Frankfurt a.M.: Campus, S. 37-50.

Ladwig, Bernd (2004): Gerechtigkeit, in: Gerhard Göhler, Mattias Iser, Ina Kerner (Hrsg.): Politische Theorie - 22 umkämpfte Begriffe zur Einführung. Wiesbaden: VS Verlag, S. 119-137.

Ladwig, Bernd (2007): Menschenrecht und menschliche Natur - Bausteine zu einer kritischen Theorie der Menschenrechte, in: Leviathan, Band 35, Nr. 1, S. 85-106.

Ladwig, Bernd (2009): Anthropologische Argumente in der menschenrechtlichen Moral, in: Dirk Jörke / Bernd Ladwig (Hrsg.): Politische Anthropologie - Chancen und Grenzen anthropologischer Begründungsmuster. Baden-Baden: Nomos, S. 245-271.

Ladwig, Bernd (2011a): Gerechtigkeitstheorien zur Einführung. Hamburg: Junius Verlag.

Ladwig, Bernd (2011b): Sich wichtig nehmen - Reflexionen zum Zusammenhang zwischen Würde und Menschenrechten. Unveröffentlichtes Manuskript. 
Maschke, Michael (2008): Behindertenpolitik in der Europäischen Union - Lebenssituation behinderter Menschen und nationale Behindertenpolitik in 15 Mitgliedstaaten.

Wiesbaden: VS Verlag.

Nohlen, Dieter / Rainer-Olaf Schultze (Hrsg.) (2004): Lexikon der Politikwissenschaft Theorien, Methoden, Begriffe. München: C.H. Beck.

Nussbaum, Martha (1992): Human Functioning and Social Justice - In Defense of Aristotelian Essentialism, in: Political Theory, Band 20, Nr. 2, S. 202-246.

Nussbaum, Martha (1993): Non-relative Virtues - An Aristotelian Approach, in: Dies. / Amartya Sen (Hrsg.): The Quality of Life - A Study prepared for the World Institute for Development Economics Research (WIDER) of the United Nations University. Oxford: Oxford University Press, S. 242-269.

Nussbaum, Martha (2007): Frontiers of Justice - Disability, Nationality, Species Membership. Cambridge: Harvard University Press.

Nussbaum, Martha (2008): Human Dignity and Political Entitlements, in: Human Dignity and Bioethics - Essays Commissioned by the President's Council on Bioethics.

Washington: Government Printing Office, S. 351-381.

Nussbaum, Martha / Amartya Sen (Hrsg.) (1993): The Quality of Life - A Study prepared for the World Institute for Development Economics Research (WIDER) of the United Nations University. Oxford: Oxford University Press.

Oliver, Michael (1996): Understanding Disability - From Theory to Practice. Basingstoke: MacMillan.

Parfit, Derek (1997): Equality and Priority, in: Ratio, Band 10, Heft 3, S. 202-221.

Pogge, Thomas (2010): A Critique of the Capability Approach, in: Harry Brighouse / Ingrid Robeyns (Hrsg.): Measuring Justice - Primary Goods and Capabilities. Cambridge: Cambridge University Press, S. 17-61.

Rawls, John (1971): A Theory of Justice. Cambridge: Harvard University Press.

Rawls, John (2003) [2001]: Justice as Fairness - A Restatement. Cambridge: Harvard University Press.

Rawls, John (2005) [1993]: Political Liberalism - Expanded Edition. New York: Columbia University Press.

Scanlon, Thomas (1975): Preference and Urgency, in: The Journal of Philosophy, Band 72, Nr. 19, S. 655-669.

Scheffler, Samuel (2003): What is Egalitarianism? in: Philosophy and Public Affairs, Band 31, Nr.1, S. 5-39.

Schramme, Thomas (2003a): Behinderung - Absolute oder relative Einschränkung des Wohlergehens? in: Ethik in der Medizin, Band 15, Nr. 3, S. 180-190. 
Schramme, Thomas (2003b): Psychische Behinderung - Natürliches Phänomen oder soziales Konstrukt? in: Günther Cloerkes (Hrsg.): Wie man behindert wird - Texte zur Konstruktion einer sozialen Rolle und zur Lebenssituation betroffener Menschen. Heidelberg: Universitätsverlag Winter, S. 53-81.

Schramme, Thomas (2006): Gerechtigkeit und soziale Praxis. Frankfurt a.M.: Campus.

Sen, Amartya (1980): Equality of What? in: Sterling M. McMurrin (Hrsg.): Tanner Lectures of Human Values, Vol. 1. Cambridge: Cambridge University Press. S. 197-220.

Sen, Amartya (1992): Inequality Reexamined. Cambridge: Harvard University Press.

Sen, Amartya (1993): Capability and Well-Being, in: Martha Nussbaum / Amartya Sen (Hrsg.): The Quality of Life - A Study prepared for the World Institute for

Development Economics Research (WIDER) of the United Nations University. Oxford: Oxford University Press, S. 30-53.

Sen, Amartya (2004): Disability and Justice, Rede anlässlich der "World Bank Disability and Inclusive Development Conference”, zugänglich unter: http://siteresources.worldbank.org/disability/214576-1092421729901/20291152/Amartya_Sen_Speech.doc, zuletzt eingesehen: 12.09.2012.

Sen, Amartya (2010): The Place of Capability in a Theory of Justice, in: Harry Brighouse / Ingrid Robeyns (Hrsg.): Measuring Justice - Primary Goods and Capabilities. Cambridge: Cambridge University Press, S. 239-254.

Shakespeare, Tom (2006): Disability Rights and Wrongs. Oxon: Routledge.

Shakespeare, Tom (2012): Disability in Developing Countries, in: Nick Watson / Alan Roulstone / Carol Tomas (Hrsg.): Handbook of Disability Studies. Oxon: Routledge, S. 271-285.

Strassenberger, Grit (2009): Mitleid und Gerechtigkeit - Martha Nussbaums anthropologische Begründung von Gerechtigkeitsnormen, in: Dirk Jörke / Bernd Ladwig (Hrsg.):

Politische Anthropologie - Chancen und Grenzen anthropologischer Begründungsmuster. Baden-Baden: Nomos, S. 129-147.

Tugendhat, Ernst (1993): Vorlesungen über Ethik. Frankfurt a.M.: Suhrkamp Verlag.

UN (2006): Übereinkommen über die Rechte von Menschen mit Schädigungen, zugänglich unter: http://www.un.org/depts/german/gv-61/band1/ar61106.pdf, zuletzt eingesehen: 12.09.2012.

UN DISTAT (2011): The United Nations Disability Statistics Database, zugänglich unter: http:/unstats.un.org/unsd/demographic/sconcerns/disability/disab2.asp, zuletzt eingesehen: 12.09 .2012 . 
Vehmas, Simo / Mäkelä, Pekka (2009): The Ontology of Disability and Impairment: A Discussion of the natural and social Features, in: Kristjana Kristiansen / Simo Vehmas / Tom Shakespeare (Hrsg.): Arguing about Disability - Philosophical Perspectives. Oxon: Routledge, S.42-57.

Vehmas, Simo (2012): What can Philosophy tell us about Disability? in: Nick Watson / Alan Roulstone / Carol Tomas (Hrsg.): Handbook of Disability Studies. Oxon: Routledge, S. 298-310.

Wasserman, David (1998): Distributive Justice, in: Anita Silvers / David Wasserman/ Mary Mahowald (Hrsg.): Disability, Difference, Discrimination - Perspectives on Justice in Bioethics and Public Policy. Lanham: Rowman and Littlefield, S. 147-209.

Wasserman, David (2001): Philosophical Issues in the Definition and Social Response to Disability, in: Gary Albrecht / Katherine Seelman / Michael Bury (Hrsg.): Handbook of Disability Studies. Thousand Oaks: Sage, S. 219-252.

Wasserman, David / Bickenbach, Jerome / Wachbroit, Robert (2005): Quality of Life and Human Difference - Genetic Testing, Health Care, and Disability. New York:

Cambridge University Press.

Wasserman, David / Asch, Adrienne / Blustein, Jeffrey / Putnam, Daniel (2011): Disability - Definitions, Models, Experience, in: Stanford Encyclopedia of Philosophy, zugänglich unter: http: //plato.stanford.edu/entries/disability, zuletzt eingesehen: 18.07.2012.

WHO (2001): International Classification of Functioning, Disability, and Health, zugänglich unter: http://www.who.int/classifications/icf/en; zuletzt eingesehen: 12.09.2012.

WHO (2011): World Report on Disability, zugänglich unter: http://www.who.int/disabilities/ world report/2011/en/index.html, zuletzt eingesehen: 12.09.2012.

Wolff, Jonathan (2009): Disability among Equals, in: Kimberley Brownlee / Adam Cureton (Hrsg.): Disability and Disadvantage. Oxford: Oxford University Press, S. 112-137.

Zola, Irving (1989): Toward the necessary Universalizing of a Disability Policy, in:

The Milbank Quarterly, Band 67, Beiheft 2, Teil 2, S. 401-428. 